\title{
The underlying neurobiology of key functional domains in young people with mood and anxiety disorders: a systematic review
}

\author{
Frank lorfino, lan B. Hickie, Rico S. C. Lee, Jim Lagopoulos and Daniel F. Hermens
}

\begin{abstract}
Background: Mood and anxiety disorders are leading causes of disability and mortality, due largely to their onset during adolescence and young adulthood and broader impact on functioning. Key factors that are associated with disability and these disorders in young people are social and economic participation (e.g. education, employment), physical health, suicide and self-harm behaviours, and alcohol and substance use. A better understanding of the objective markers (i.e. neurobiological parameters) associated with these factors is important for the development of effective early interventions that reduce the impact of disability and illness persistence.

Methods: We systematically reviewed the literature for neurobiological parameters (i.e. neuropsychology, neuroimaging, sleep-wake and circadian biology, neurophysiology and metabolic measures) associated with functional domains in young people (12 to 30 years) with mood and/or anxiety disorders.

Results: Of the one hundred and thirty-four studies selected, $7.6 \%$ investigated social and economic participation, 2. $1 \%$ physical health, $15.3 \%$ suicide and self-harm behaviours, $6.9 \%$ alcohol and substance use, whereas the majority (68.1\%) focussed on clinical syndrome.

Conclusions: Despite the predominance of studies that solely examine the clinical syndrome of young people the literature also provides evidence of distinct associations among objective measures (indexing various aspects of brain circuitry) and other functional domains. We suggest that a shift in focus towards characterising the mechanisms that underlie and/or mediate multiple functional domains will optimise personalised interventions and improve illness trajectories.
\end{abstract}

Keywords: Depression, Anxiety, Bipolar, Functional outcomes, Biomarkers, Neurobiology, Neuropsychology, Personalised psychiatry

\section{Background}

Depression and anxiety are associated with the greatest burden of disease of all neurological, psychiatric and substance use disorders [1]. The early onset of psychiatric illness plays a key role in such disability with approximately $75 \%$ of these disorders occurring before the age of 25 years [2]. Despite this, our current capacity to provide tailored early interventions and prevent the progression of illness or slow the pathway to disability is lacking [3].

\footnotetext{
* Correspondence: daniel.hermens@sydney.edu.au

Clinical Research Unit, Brain and Mind Centre, University of Sydney, 94 Mallet

Street, Camperdown, NSW 2050, Australia
}

Whilst, some specific diagnoses have been successfully treated with certain interventions (e.g. CBT for social anxiety disorder; [4]), there are limitations to the optimal treatment for unipolar, bipolar and comorbid mood disorders. This is particularly evident for those with emerging illnesses who often experience mixed states and/or subthreshold symptoms [5]. Since these mood states typically arise during adolescence and young adulthood, a period of critical brain development and functional independence, the impact of illness can lead to greater disability and worse illness outcomes [6-8]. Thus, the search for objective 
markers of early risk states with predicative capacity in regards to disability and mortality requires rigorous investigation so that appropriate interventions can be trialled and delivered as early as possible to reduce the impact of disability $[3,9]$.

Traditionally, there has been a focus on the 'clinical syndrome' defined as identifying distinct clinical categories or disorders (based on ICD or DSM diagnostic criteria) with specific thresholds and the impact of these on functioning. However, even at a subthreshold symptom level significant contributors to disability and mortality include social and economic disability $[10,11]$, poor physical health (e.g. diabetes) [12], high suicide and self-harm behaviours [10, 13-15], and risky alcohol and substance use [16, 17]. Given the clinical impact of these factors for young people with emerging mood and anxiety disorders, we have identified them as key functional domains that, we argue, should be the focus of targeted personalised assessment and intervention. Although the term 'functional domain' has traditionally, often referred to outcomes relating to occupational (i.e. employment and education) status, here, we use the term to include other key factors that have significant (often concomitant) impacts on levels of functioning in young people. These domains largely align with the framework provided by the 'International classification of functioning, disability and health' [18] for conceptualising health and health related states. These include: (i) social and economic participation (i.e. engagement and stability in employment, education and social relationships); (ii) physical health; (iii) suicide and self-harm behaviours; (iv) alcohol and substance use; and (v) clinical syndrome (i.e. diagnostic category, stage of illness and severity of symptoms [3]). These domains are priority areas for service models in Australia (e.g. headspace $[19,20])$, which recognise the need for early interventions that aim to target specific outcomes associated with illness persistence and greater disability [21]. Importantly, a focus on these five domains recognises the need to evaluate multiple (often interacting) aspects of an individual to better characterise their specific phenotype and, as a result, attempt to predict their potential illness trajectory.

To overcome some of the limitations associated with current diagnostic approaches that link poorly to neurobiological risk factors or patterns of treatment response it is important to characterise the neurobiology that may underlie or mediate observable functional impairment(s) $[9,22]$. This emphasises the need to focus on the four remaining functional domains in addition to the traditional focus on the clinical syndrome to optimise personalised interventions. Models of psychopathology suggest that breakdowns in common brain circuits involved in cognition and behaviour are responsible for the development of psychopathology and general dysfunction [23]. In this view, quantifying the integrity of such brain systems (e.g. via neuroimaging, neurophysiology or circadian biology) along with their behavioural concomitants (e.g. neuropsychology, social cognition or sleep-wake patterns) may lead to the identification of objective markers of early risk states and also serve as treatment targets. For example, in a longitudinal study by our group, neuropsychological performance at baseline was the single best predictor of sociooccupational functioning at follow up, over and above diagnosis and symptom severity [24]. Such findings demonstrate the relevance of objective 'brain' markers (in this case, a cognitive phenotype) to provide important insights about a crucial functional domain, which cut across diagnostic categories to direct effective treatment strategies at the pathophysiological driver of poor patient outcomes.

Here, we present a systematic review of the neurobiological and neurocognitive correlates, of five functional domains in young people with mood and anxiety disorders. We focus specifically on major depression, bipolar disorder (I, II, not otherwise specified; NOS) and anxiety disorders (excluding posttraumatic stress disorder), since these most closely relate to the common developmental trajectories of emerging mood disorders in young people [25]. In this review we evaluate the relationship between the functional domains (described above), and evidence from neuropsychology, neuroimaging, sleep-wake and circadian biology, neurophysiology and metabolic studies. A wide age range was chosen (12-30 years) to focus on the adolescent and young adult population; referred to collectively as 'young people', to better understand the primary age group that are vulnerable and present to primary youth mental health services. The primary objective of this study is to establish the current status of the literature of young people with mood and anxiety disorder with respect to neurobiological investigations addressing any of the proposed five functional domains. Whilst, we expect that the large majority of identified studies would investigate clinical syndromes and a smaller number would investigate the remaining functional domains, it is expected that unique associations between neurobiological parameters and a functional domain, not accounted for by the clinical syndrome, will become clearer. The aim of our approach is to ultimately provide a framework for guiding the development of personalised assessment and interventions to prevent or delay significant disability in young mental health patients. 


\section{Methods}

Methods of review regarding eligibility criteria, data collection and synthesis were specified in advance in the form of a review protocol. We followed the guidelines for conducting and reporting a systematic review set out by 'the PRISMA statement' [26], and the 'Cochrane Handbook for Systematic Reviews' [27].

\section{Eligibility criteria \\ Report characteristics and information sources}

We searched PubMed databases for unique records using the following criteria: (i) published in the last 20 years (i.e. between January 1994 and March, 2014, to coincide with the release of DSM-IV since this version introduced the use of clinical significance ratings related to the impact of illness on areas of functioning); (ii) the study was reported in English; and (iii) had keyword combinations (see Table 1 for full search terms). The reference lists of studies identified by our PubMed search were not utilised as an additional information source.

\section{Study characteristics and selection}

Using a pro forma, the first author (FI) checked the abstract and/or full texts of each paper for the following inclusion criteria; i) a mean age between 12 and 30 years; ii) at least one group of subjects was reported as having a primary mood and anxiety (i.e. depression, bipolar, anxiety) disorder (according to DSM-IV or ICD-10 criteria) or syndrome (e.g. 'at risk', current depressive symptoms); iii) at least one of the following functional domains: (a) social and economic participation; (b) physical health; (c) suicide and selfharm behaviours; (d) alcohol and substance use; and/ or (e) clinical syndrome, was measured/quantified; and iv) a statistical (i.e. correlational, regression, etc.) association between the functional domain and at least one neurobiological parameter (i.e. neuropsychology, brain imaging, sleep-wake and circadian biology, neurophysiology and metabolic) was reported. Review articles and case studies were excluded from the final synthesis. Studies were labelled 'yes' if they fulfilled all four criteria, 'no' if they failed to meet all four criteria or 'possibly' if it was unclear whether all

Table 1 Full list of search terms used according to each topic area

\begin{tabular}{|c|c|c|}
\hline & Topic area & Pub Med Terms \\
\hline \multirow[t]{2}{*}{ Population of interest } & Mood and anxiety syndrome or profile & $\begin{array}{l}\text { Anxiety disorder OR anxiety OR depression OR depressive } \\
\text { disorders OR depressive disorder [MeSH Terms] OR major } \\
\text { depressive disorder OR MDD OR disorder, bipolar } \\
\text { [MeSH Terms] OR bipolar disorder OR affective disorder OR } \\
\text { mood disorder OR affective syndrome OR manic syndrome } \\
\text { OR depressive syndrome OR anxious syndrome }\end{array}$ \\
\hline & Youth & $\begin{array}{l}\text { Adolescents OR young people OR adolescence OR adolescent } \\
\text { [MeSH Terms] OR youth OR young adult }\end{array}$ \\
\hline \multirow[t]{5}{*}{ Functional domain } & Social and economic participation & $\begin{array}{l}\text { Socio-occupational functioning OR functioning OR social } \\
\text { functioning OR occupational participation OR economic } \\
\text { participation }\end{array}$ \\
\hline & Physical health & $\begin{array}{l}\text { Physical health OR metabolic rate OR obesity OR blood } \\
\text { pressure OR CVD OR fitness OR cardiovascular disease OR } \\
\text { BMI or body mass index OR waist measurement OR blood } \\
\text { glucose OR smoking rate OR physical activity OR cholesterol } \\
\text { levels }\end{array}$ \\
\hline & Suicide and self-harm behaviours & $\begin{array}{l}\text { Suicide [MeSH Terms] OR suicide ideation OR self-harm OR } \\
\text { suicide risk }\end{array}$ \\
\hline & Alcohol and substance use & $\begin{array}{l}\text { Substance use disorder [MeSH Terms] OR alcohol use OR } \\
\text { drug use }\end{array}$ \\
\hline & Clinical syndrome & $\begin{array}{l}\text { Illness progression OR syndrome progression OR symptom } \\
\text { severity }\end{array}$ \\
\hline \multirow[t]{5}{*}{ Neurobiological parameter } & Neuropsychology & Neuropsychology OR neuropsychological test [MeSH Terms] \\
\hline & Imaging & $\begin{array}{l}\text { Brain imaging [MeSH Terms] OR imaging OR neuroimaging } \\
\text { OR fMRI OR DTI OR MRI OR MRS }\end{array}$ \\
\hline & Sleep-wake and circadian biology & $\begin{array}{l}\text { Actigraphy [MeSH Terms] OR melatonin secretion OR } \\
\text { circadian rhythms OR DLMO OR sleep-wake and circadian } \\
\text { biology }\end{array}$ \\
\hline & Neurophysiology & $\begin{array}{l}\text { Neurophysiology OR EEG OR electroencephalography OR } \\
\text { ERP OR event-related potentials }\end{array}$ \\
\hline & Metabolic & BMI OR waist measurement OR blood pressure OR cholesterol \\
\hline
\end{tabular}


criteria were fully met. Any disagreement with these rules was resolved by consensus with the senior author $(\mathrm{DH})$.

\section{Identification of studies}

Figure 1 displays the series of steps undertaken as we identified studies for this systematic review. First, of the 3975 studies identified by the searches (see Table 1 for search terms), 565 titles and abstracts were examined for eligibility. At this stage, 188 studies were excluded on the basis of not meeting one or more of the eligibility criteria specified. The eligibility stage involved the assessment 377 full texts (i.e. the published manuscript) to evaluate whether these studies were suitable, which led to a further 243 studies being excluded. The remaining 134 studies were included in the final synthesis (see Table 2 for a summary of these studies).

\section{Synthesis of results}

For each of the included studies, the reviewer (FI) collated data with respect to the study design (i.e. crosssectional, longitudinal; see Table 2), sample characteristics (i.e. age, sample sizes), aims, key measures (e.g. neuropsychological, circadian, clinical) and key findings (presented in Tables 3, 4, 5, 6 and 7; one table per neurobiological parameter). To clarify, the key findings for each study were taken as any evidence of an association between a particular neurobiological measure and a functional domain. In order to achieve this, the various scales, tests, and assessments were collapsed into broader categories of key measures (e.g. specific neuropsychological subtests grouped into a cognitive domain; see Tables 3, 4, 5, 6 and 7). Given the variability in methodology and the large and varied outcomes of identified studies it was not appropriate to carry out a metaanalysis [28].

\section{Results}

A total of 134 studies were included in this systematic review (see Fig. 1); 10 of these studies were featured more than once in the data synthesis to make a total of 144 reported results. As summarised in Table 2, the included studies were categorized according to functional domain in the following proportions: $7.6 \%(k=11)$ investigated social and economic participation, $2.1 \%(k=$ 3) physical health, $15.3 \%(k=22)$ suicide and self-harm behaviours, $6.9 \%(k=10)$ alcohol and substance use, and $68.1 \%(k=98)$ clinical syndrome. In regards to neurobiological parameters, $19.4 \%(k=28)$ focused on neuropsychology, $43.1 \%(k=62)$ on neuroimaging, $16 \%$ $(k=23)$ on sleep-wake and circadian biology, $14.6 \%(k=$ 21) on neurophysiology and $6.9 \%(k=10)$ on metabolic measures. The range of the mean ages for patient groups

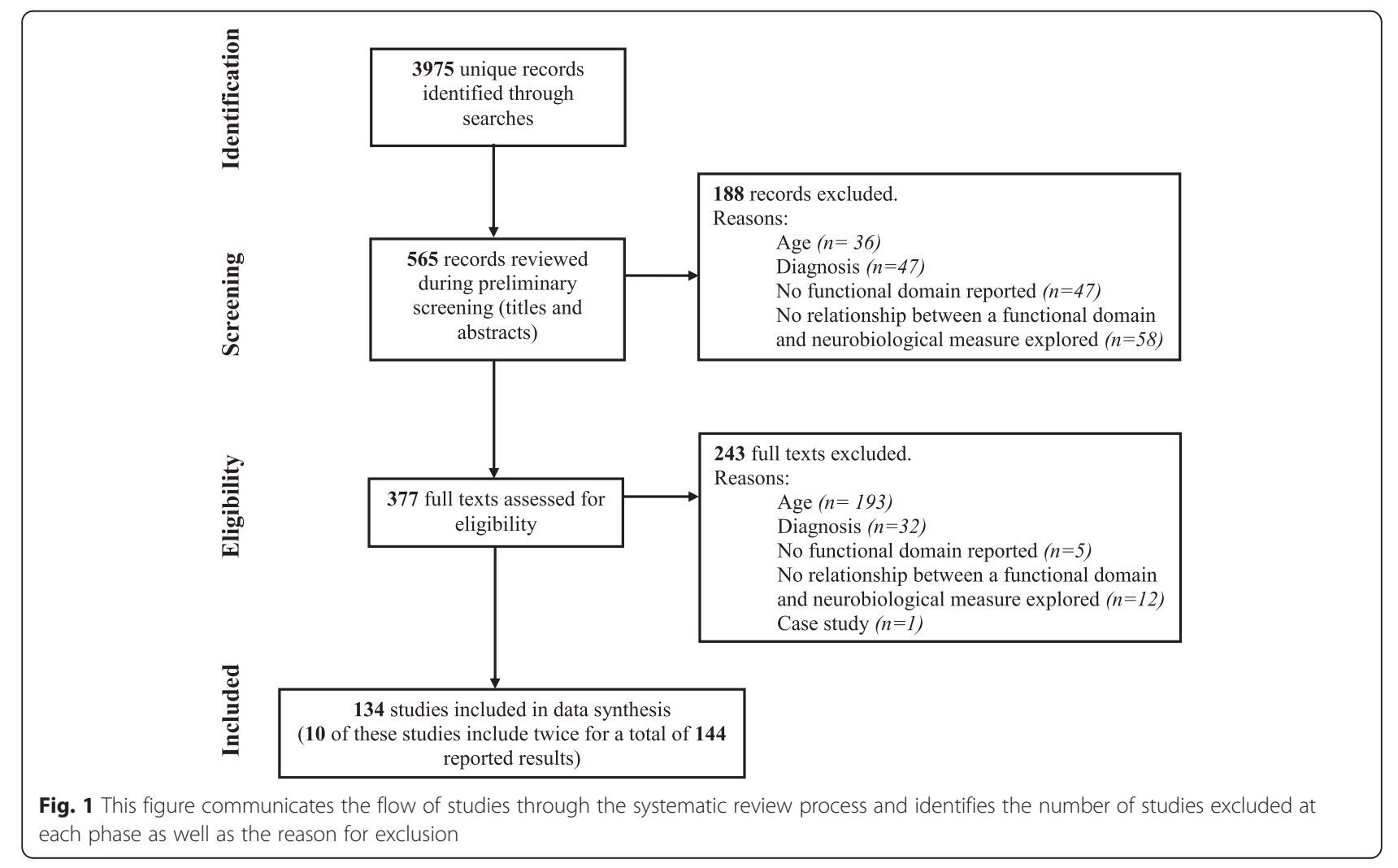


Table 2 Overview of the included studies organised according to functional domain and neurobiological parameter investigated

\begin{tabular}{|c|c|c|c|c|c|}
\hline \multirow[b]{2}{*}{ Functional domain } & \multicolumn{5}{|c|}{ Neurobiological parameter } \\
\hline & Neuropsychology & Imaging & Sleep-wake and circadian biology & Neurophysiology & Metabolic \\
\hline \multirow[t]{5}{*}{ Social \& economic participation } & Beiderman (2011) & Perlman (2012) & Abelson (1996) & Kaur (2013) & Taylor $(2008)^{*}$ \\
\hline & Fujii $(2013)^{*}$ & & Goodyer $(1998)^{*}$ & & \\
\hline & Korhonen (2002) & & Granger (1994) & & \\
\hline & Lee (2013c) & & & & \\
\hline & Lee $(2013 a)$ & & & & \\
\hline Physical health & & Bond (2011) & & Jarworska $(2011)^{*}$ & Mannie (2013) \\
\hline \multirow[t]{6}{*}{ Suicide \& self-harm behaviours } & Bridge (2012) & Ehrlich (2004) & Coplan $(2000)^{*}$ & Ashton (1994) & Apter (1999) \\
\hline & Miranda (2012) & Ehrlich (2005) & Mathew (2003) & Graae (1996) & De Berardis (2013) \\
\hline & Ohmann (2008) & Goodman $(2011)^{*}$ & McCracken (1997)* & Pechtel (2013) & Plana (2010) \\
\hline & Oldershaw (2009) & Pan (2011) & & & Soreni (1999) \\
\hline & $\operatorname{Pan}(2013 b)^{*}$ & Pan (2013a) & & & Tyano (2006) \\
\hline & & Pan $(2013 b)^{*}$ & & & \\
\hline \multirow[t]{5}{*}{ Alcohol \& substance use } & Harvey (2007) & Chitty (2013) & & Chitty (2014) & Goldstein (2008) \\
\hline & Hermens (2013a) & Cornelius (2010) & & Ehlers (2011) & \\
\hline & & De Bellis (2005) & & & \\
\hline & & Jarvis (2008) & & & \\
\hline & & Medina (2007) & & & \\
\hline \multirow[t]{25}{*}{ Clinical syndrome } & Andres (2007) & Adler (2007) & Adam (2010) & Bakker (2011) & Pine (2001) \\
\hline & Andres (2008) & Aghajani (2013) & Ankers (2009) & Carrasco (2013a) & Taylor $(2008)^{*}$ \\
\hline & Basso (2001) & Bitter (2011) & Armitage (1997) & Carrasco (2013b) & \\
\hline & Cataldo (2005) & Chang (2008) & Coplan $(2000)^{*}$ & Croarkin (2014) & \\
\hline & Fleck (2008) & Chu (2013) & Doane (2013) & Dai (2012) & \\
\hline & Fujii $(2013)^{*}$ & Diler (2013) & Ellenbogen (2006) & El Badri (2001) & \\
\hline & Gunther (2004) & Forbes (2006) & Ellenbogen (2010) & Hajcak (2008) & \\
\hline & Han (2012) & Forbes (2010) & Goodyer (1998)* & Houston (2003) & \\
\hline & KIVIrCIK $(2003)^{*}$ & Gabbay (2012) & Harkness (2011) & Jarworska $(2011)^{*}$ & \\
\hline & Klimkeit (2011) & Gabbay (2013) & Landsness (2011) & Jarworska (2013) & \\
\hline & Okasha $(2000)^{*}$ & Gao (2013) & McCracken (1997)* & KIvırcık $(2003)^{*}$ & \\
\hline & Pavuluri (2010a) & Gilbert (2000) & Murray (2012) & Okasha $(2000)^{*}$ & \\
\hline & Schmid (2013) & Gilbert (2009) & Rao (1996) & Stern (2010) & \\
\hline & Simons (2009) & Goodman $(2011)^{*}$ & Rao (2008) & Vaidyanathan (2014) & \\
\hline & Torres (2010) & Gruner (2012) & Robillard (2013a) & & \\
\hline & Wall (2013) & Hatton (2012) & Robillard (2013b) & & \\
\hline & & Henderson (2013) & Scott (2014) & & \\
\hline & & Ho (2014) & & & \\
\hline & & Huang (2012) & & & \\
\hline & & Huyser (2011) & & & \\
\hline & & Huyser (2013) & & & \\
\hline & & Ladouceur (2011) & & & \\
\hline & & Lagopoulos (2012) & & & \\
\hline & & Lagopoulos (2013a) & & & \\
\hline & & Lagopoulos (2013b) & & & \\
\hline
\end{tabular}


Table 2 Overview of the included studies organised according to functional domain and neurobiological parameter investigated (Continued)

\begin{tabular}{|c|c|c|c|c|c|}
\hline \multirow[b]{2}{*}{ Functional domain } & \multicolumn{5}{|c|}{ Neurobiological parameter } \\
\hline & Neuropsychology & Imaging & Sleep-wake and circadian biology & Neurophysiology & Metabolic \\
\hline \multirow[t]{23}{*}{ Clinical syndrome } & & Lazaro (2012) & & & \\
\hline & & Lisy (2011) & & & \\
\hline & & MacMaster (2006) & & & \\
\hline & & MacMillian (2003) & & & \\
\hline & & McClure (2007) & & & \\
\hline & & Meng (2013) & & & \\
\hline & & Pannekoek (2014) & & & \\
\hline & & Patel (2008) & & & \\
\hline & & Pavuluri (2010b) & & & \\
\hline & & Pavuluri (2011) & & & \\
\hline & & Phan (2013) & & & \\
\hline & & Rauch (2002) & & & \\
\hline & & Reynolds (2014) & & & \\
\hline & & Rosenberg (1997) & & & \\
\hline & & Rosenberg (2000) & & & \\
\hline & & Rosso (2005) & & & \\
\hline & & Schienle (2011) & & & \\
\hline & & Schneider (2012) & & & \\
\hline & & Strawn (2012) & & & \\
\hline & & Wegbreit (2011) & & & \\
\hline & & Yucel (2008) & & & \\
\hline & & Zarei (2011) & & & \\
\hline & & Zuo (2013) & & & \\
\hline
\end{tabular}

Note. ${ }^{*}=$ indicates the study appears more than once, BOLD = longitudinal study, italicized = Study conducted by the Brain and Mind Centre

across all the included studies was 11.7 to 31.7 years, since those studies that had comparison groups both inside and outside the inclusion criteria of 12 to 30 were still included.

\section{Neuropsychology}

There were 28 studies (a total of 2877 participants; $58.5 \%$ female) that utilised neuropsychology and across these studies $69 \%(2037 / 2877)$ were patients and $29 \%$ $(804 / 2877)$ were healthy controls. Among the patient group $47 \%$ (966/2037) had depression, $15 \%(301 / 2037)$ had bipolar, $12 \%(239 / 2037)$ had anxiety, and $26 \%$ (531/2037) were classified as other.

Functional domains: social and economic participation, physical health, suicide and self-harm \& alcohol and substance use

Our systematic search found an association between neuropsychology and three functional domains (i.e. social and economic participation, suicide and self-harm and alcohol and substance use). No studies that met our criteria investigated physical health. The relationship between global deficits in cognition and social and economic participation in mood disorders is unclear as there were only two studies; one reporting a positive relationship [24], and the other no relationship [29]. The former study utilised a mixed psychiatric sample that consisted of mood disorder and psychosis patients, which may have influenced the results given the well-supported relationship between social and economic participation and neuropsychology in patients with psychosis [30]. However, the latter study only investigated MDD $(N=16)$ and utilised the Global Assessment of Functioning scale (GAF) as a measure of participation, which could be problematic as the rating can be made on the basis of symptoms or functioning. Thus, it is clear that more studies are needed in this population to resolve or clarify such findings.

Studies exploring specific neuropsychological capabilities have provided greater insight into how these relate to functional domains that can be ambiguous when investigating global cognition. Of the neuropsychological studies reviewed, executive function appears to be 
Table 3 Neuropsychological studies evaluating the five functional domains in young people (12-30 yrs) with a mood and/or anxiety disorder

\begin{tabular}{|c|c|c|c|c|c|c|}
\hline $\begin{array}{l}\text { Outcome } \\
\text { measure }\end{array}$ & Study & Age (mean $\pm S D)$ & Sample (N) & Aims & Key measures & Key findings \\
\hline \multirow[t]{5}{*}{$\begin{array}{l}\text { Social and } \\
\text { economic } \\
\text { participation }\end{array}$} & {$[40]$} & $\begin{array}{l}\text { HC: } 13.6 \pm 2.1 \\
\text { HC-EFD: } 13.9 \pm 2.3 \\
\text { BPD-I: } 13.7 \pm 2.1 \\
\text { BPD-I-EFD: } 12.8 \pm 2.4\end{array}$ & $\begin{array}{l}\text { HC (47M; 34F), } \\
\text { HC-EFD (12M; 5F), } \\
\text { BPD-I (52M; 24F), } \\
\text { BPD-I-EFD (49M; 13F) }\end{array}$ & $\begin{array}{l}\text { Evaluate the clinical } \\
\text { impact of executive } \\
\text { function deficits in youth } \\
\text { with BPD-I disorder. }\end{array}$ & $\begin{array}{l}\text { NY: Executive function } \\
\text { deficits (CPT, CVLT-C, RCF, } \\
\text { SCWT, WCST, WAIS-III- } \\
\text { FFD) }\end{array}$ & $\begin{array}{l}\text { BPD-I: } \downarrow \text { executive } \\
\text { function } \sim \downarrow \text { social and } \\
\text { economic participation }\end{array}$ \\
\hline & {$[39]^{*}$} & $\begin{array}{l}\text { SAD: } 23.9 \pm 6.7 \\
H C: 25.6 \pm 5.6\end{array}$ & SAD (20M; 10F) & $\begin{array}{l}\text { Assess the } \\
\text { neuropsychological } \\
\text { function of SAD without } \\
\text { co-morbidity }\end{array}$ & $\begin{array}{l}\text { NU: Executive function } \\
\text { (CPT, TMT-B, WCST), Pro- } \\
\text { cessing speed (TMT-A), } \\
\text { Verbal learning \& mem- } \\
\text { ory (AVLT) } \\
\text { Functional: GAF }\end{array}$ & $\begin{array}{l}\text { SAD: } \downarrow \text { executive } \\
\text { function } \sim \downarrow \text { social and } \\
\text { economic participation } \\
\text { (and } \uparrow \text { SAD severity) }\end{array}$ \\
\hline & \multirow[t]{2}{*}[29]{} & $\begin{array}{l}\text { MDD: } 18.9 \pm 2.0 \\
\text { HC: } 16.9 \pm 1.9\end{array}$ & $\begin{array}{l}\text { MDD (4M; 12F), } \\
\text { HC (11M; 14F) }\end{array}$ & $\begin{array}{l}\text { Investigate the } \\
\text { association between } \\
\text { cognitive performance } \\
\text { and MDD. }\end{array}$ & $\begin{array}{l}\text { NU: Executive function } \\
\text { (SCWT, TMT-B), Verbal } \\
\text { learning \& memory } \\
\text { (WMS-SR, LLT, RCF-3min), } \\
\text { General intellect (WAIS-III- } \\
\text { S \& V), Attention (WAIS-III- } \\
\text { DS, BD \& DSp) }\end{array}$ & $\begin{array}{l}\text { MDD: } \downarrow \text { social and } \\
\text { economic participation }\end{array}$ \\
\hline & & $\begin{array}{l}\text { FED: } 22.00 \pm 4.9 \\
\text { FEP: } 23.30 \pm 3.9\end{array}$ & FEP (20M; 13F) & $\begin{array}{l}\text { Assess the effectiveness } \\
\text { of CR in patients with a } \\
\text { first-episode of either } \\
\text { major depression or } \\
\text { psychosis }\end{array}$ & $\begin{array}{l}\text { NY: Executive function } \\
\text { (CANTAB-IED; -FAS, TMT- } \\
\text { B), Processing speed } \\
\text { (TMT-A, category fluency), } \\
\text { Attention and working } \\
\text { memory (LDSF, LDSB, } \\
\text { CANTAB-SSP;-RVP, mental } \\
\text { control), Immediate learn- } \\
\text { ing and memory (Logical } \\
\text { Memory I, RAVLT- tot, } \\
\text { CANTAB-PAL), Delayed } \\
\text { learning and memory } \\
\text { (LM-Ret, RCF-3min, } \\
\text { RAVLT-Ret) } \\
\text { Functional: SFS }\end{array}$ & $\begin{array}{l}\text { FED \& FEP: CR } \uparrow \\
\text { immediate learning and } \\
\text { memory, and } \uparrow \text { social } \\
\text { and economic } \\
\text { participation (mediated } \\
\text { by } \uparrow \text { delayed learning } \\
\text { and memory) }\end{array}$ \\
\hline & {$[24]$} & MHP: $21.6 \pm 4.5$ & $\begin{array}{l}\text { PSD (30) } \\
\text { (of the total 93, } \\
52 \% \text { were male) }\end{array}$ & $\begin{array}{l}\text { Identify cognitive markers } \\
\text { that predict later socio- } \\
\text { occupational functioning. }\end{array}$ & $\begin{array}{l}\text { NU : Executive function } \\
\text { (CANTAB-IED, TMT-B), Pro- } \\
\text { cessing speed (TMT-A, } \\
\text { CANTAB-FAS), Attention } \\
\text { and working memory } \\
\text { (CANTAB-RVP), Verbal } \\
\text { learning \& memory (LM- } \\
\text { Ret, RAVLT-ret), Visual } \\
\text { learning \& memory (CAN- } \\
\text { TAB- SSP;- PAL) }\end{array}$ & $\begin{array}{l}\text { MHP: } \uparrow \text { BL general N } \Psi \\
\sim \uparrow \text { social and economic } \\
\text { participation at FUP }\end{array}$ \\
\hline \multirow[t]{3}{*}{$\begin{array}{l}\text { Suicide and } \\
\text { self-harm }\end{array}$} & {$[31]$} & SA: $15.5 \pm 1.4$ & SA (10M; 30F) & $\begin{array}{l}\text { Examine decision-making } \\
\text { processes in suicide } \\
\text { attempters and never- } \\
\text { suicidal comparison } \\
\text { subjects }\end{array}$ & $\begin{array}{l}\text { NY: Decision making } \\
(\mathrm{IGT})\end{array}$ & $\begin{array}{l}\text { SA: } \downarrow \text { decision making } ~ \\
\text { suicide attempt history }\end{array}$ \\
\hline & {$[32]$} & NSA: $18.31 \pm 0.78$ & NSA (9M; 23F) & $\begin{array}{l}\text { Examine whether } \\
\text { cognitive inflexibility can } \\
\text { differentially and } \\
\text { prospectively predict } \\
\text { suicidal ideation. }\end{array}$ & $\begin{array}{l}\text { N } \Psi \text { : Executive function } \\
\text { (WCST; perseverative } \\
\text { errors) }\end{array}$ & $\begin{array}{l}\text { SA: } \downarrow \text { BL cognitive } \\
\text { inflexibility } \sim \uparrow \text { suicide } \\
\text { ideation at 6-month FUP. }\end{array}$ \\
\hline & [35] & $\begin{array}{l}\text { SIB: } 15.5 \pm 1.3 \\
\text { NSIB: } 15.1 \pm 1.4\end{array}$ & $\begin{array}{l}\text { SIB (99) } \\
\operatorname{NSIB~(77)~}\end{array}$ & $\begin{array}{l}\text { Investigate the } \\
\text { neuropsychological } \\
\text { differences between }\end{array}$ & $\begin{array}{l}\text { NY: Executive function } \\
\text { (SCWT, WCST) }\end{array}$ & Null findings \\
\hline
\end{tabular}


Table 3 Neuropsychological studies evaluating the five functional domains in young people (12-30 yrs) with a mood and/or anxiety disorder (Continued)

\begin{tabular}{|c|c|c|c|c|c|c|}
\hline & & & & $\begin{array}{l}\text { psychiatric patients with } \\
\text { and without SIB. }\end{array}$ & $\begin{array}{l}\text { Functional: Clinical } \\
\text { interview }\end{array}$ & \\
\hline & \multirow[t]{3}{*}{ [33] } & HC: $15.8 \pm 1.5$ & $\mathrm{HC}(11 \mathrm{M} ; 46 \mathrm{~F})$ & \multirow{3}{*}{$\begin{array}{l}\text { Assess decision making } \\
\text { and problem solving } \\
\text { ability in adolescents with } \\
\text { current or past self-harm }\end{array}$} & $\begin{array}{l}\text { NU: Decision making (IGT, } \\
\text { MEPS) }\end{array}$ & \multirow{3}{*}{$\begin{array}{l}\text { DSH: } \downarrow \text { decision making } \\
\sim \text { current, but not past } \\
\text { DSH }\end{array}$} \\
\hline & & PC: $15.7+1.3$ & PC (2M; 20F) & & Functional: Clinical & \\
\hline & & DSH: $15.8+1.5$ & DSH (5M; 49F) & & & \\
\hline & \multirow[t]{3}{*}[34]{$^{*}$} & SA: $16.20 \pm 0.78$ & $\mathrm{SA}(4 \mathrm{M} ; 11 \mathrm{~F})$ & \multirow{3}{*}{$\begin{array}{l}\text { Measure neural activity } \\
\text { during performance on } \\
\text { the IGT in adolescents. }\end{array}$} & $\begin{array}{l}\mathrm{N} \Psi \text { : Decision making } \\
(\mathrm{IGT}-\mathrm{mod})\end{array}$ & \multirow[t]{3}{*}{$\begin{array}{l}\text { SA: } \uparrow \text { decision making } \\
\text { suicide attempt history }\end{array}$} \\
\hline & & PC: $15.79 \pm 1.58$ & PC (7M; 7F) & & Functional: C-CASA, CSHF, & \\
\hline & & HC: $15.15 \pm 1.46$ & $\mathrm{HC}(8 \mathrm{M} ; 5 \mathrm{~F})$ & & & \\
\hline \multirow[t]{8}{*}{$\begin{array}{l}\text { Alcohol and } \\
\text { substance use }\end{array}$} & \multirow[t]{3}{*}[164]{} & \multirow[t]{3}{*}{$\begin{array}{l}\text { CU: } 16.2 \\
(13.5-18.4)\end{array}$} & \multirow[t]{3}{*}{ CU (28M; 42F) } & \multirow{3}{*}{$\begin{array}{l}\text { Investigate the non-acute } \\
\text { relationship between can- } \\
\text { nabis use and cognitive } \\
\text { function }\end{array}$} & \multirow{2}{*}{$\begin{array}{l}\text { NY: Intelligence (WASI), } \\
\text { Executive function } \\
\text { (CANTAB-IED), (CANTAB- } \\
\text { MS), Attention and } \\
\text { working memory } \\
\text { (CANTAB-RVP;-SWM; -SSP, } \\
\text { DS, SDMT), Immediate } \\
\text { learning and memory } \\
\text { (RAVLT, CANTAB-PAL) }\end{array}$} & $\begin{array}{l}C U: \downarrow \text { attention, spatial } \\
\text { working memory and } \\
\text { learning. }\end{array}$ \\
\hline & & & & & & $\begin{array}{l}\text { CU: was independent } \\
\text { predictor of performance } \\
\text { on the working memory } \\
\text { and strategy measures }\end{array}$ \\
\hline & & & & & Functional: TLFB & \\
\hline & \multirow[t]{5}{*}{ [165] } & HC-NB: $22.9 \pm 3.1$ & HC-NB (7M; 14F) & \multirow{5}{*}{$\begin{array}{l}\text { Compare the cognition in } \\
\text { binge drinkers with } \\
\text { depression to those with } \\
\text { depression alone or } \\
\text { binge drinking alone. }\end{array}$} & \multirow{4}{*}{$\begin{array}{l}\text { NY: Intelligence (WTAR), } \\
\text { Psychomotor speed } \\
\text { (TMT-A) Executive } \\
\text { function (TMT-B), Verbal } \\
\text { learning and memory } \\
\text { (RAVLT), Attention } \\
\text { (CANTAB- RVP), working } \\
\text { memory (CANTAB-SSP, } \\
\text { Visuospatial learning and } \\
\text { memory (CANTAB-PAL) }\end{array}$} & \multirow{5}{*}{$\begin{array}{l}\text { MDD-B: } \downarrow \text { visual learning } \\
\& \text { memory and overall } \\
\text { pattern of } \downarrow N \Psi \\
\text { functioning. }\end{array}$} \\
\hline & & HC-B: $23.0 \pm 2.5$ & HC-B $(13 \mathrm{M} ; 11 \mathrm{~F})$ & & & \\
\hline & & MDD-NB: $21.7 \pm 3.2$ & MDD-NB (16M; 32F) & & & \\
\hline & & \multirow[t]{2}{*}{ MDD-B: $21.8 \pm 3.4$} & \multirow[t]{2}{*}{ MDD-B (24M; 19F) } & & & \\
\hline & & & & & Functional: AUDIT & \\
\hline \multirow{6}{*}{$\begin{array}{l}\text { Clinical } \\
\text { syndrome }\end{array}$} & \multirow[t]{3}{*}[50]{} & OCD: $13.84 \pm 2.78$ & OCD (18M; 17F) & \multirow{3}{*}{$\begin{array}{l}\text { Investigate the influence } \\
\text { of clinical variables } \\
\text { treatment on cognitive } \\
\text { performance in OCD } \\
\text { patients }\end{array}$} & \multirow[b]{2}{*}{$\begin{array}{l}\text { NY: Intelligence (WISC-R: } \\
\text { Vo), Visual organisation } \\
\text { (WISC-R:-BD), Attention } \\
\text { (WISC-R: -DS;-Co), Verbal } \\
\text { learning and memory } \\
\text { (WMS-III- LM1 \& 2, } \\
\text { RAVLT), Visual learning } \\
\text { and memory (WMS-III: VR } \\
1 \text { \& 2, RCFT), Processing } \\
\text { speed (TMT-A), Cognitive } \\
\text { flexibility (TMT-B, WCST, } \\
\text { SCWT), Verbal fluency } \\
\text { (COWAT) }\end{array}$} & \multirow{3}{*}{$\begin{array}{l}\text { OCD: } \downarrow \text { verbal and visual } \\
\text { memory and velocity. } \\
\text { (Neuropsychological } \\
\text { impairment was not } \\
\text { related to obsessive- } \\
\text { compulsive severity) }\end{array}$} \\
\hline & & $\mathrm{HC}: 13.81 \pm 2.74$ & HC (18M; 17F) & & & \\
\hline & & & & & Clinical: CDI, Y-BOCS & \\
\hline & \multirow[t]{3}{*}{ [51] } & OCD: $13.46 \pm 2.83$ & OCD (16M; 13F) & \multirow{3}{*}{$\begin{array}{l}\text { Explore the evolution of } \\
\text { cognitive dysfunction in } \\
\text { children and adolescents } \\
\text { with OCD after treatment }\end{array}$} & \multirow[b]{2}{*}{$\begin{array}{l}\text { NY: Intelligence (WISC-R: } \\
\text { Vo), Visual organisation } \\
\text { (WISC-R:-BD), Attention } \\
\text { (WISC-R: -DS;-Co), Verbal } \\
\text { learning and memory } \\
\text { (WMS-III- LM1 \& 2, } \\
\text { RAVLT), Visual learning } \\
\text { and memory (WMS-III: VR } \\
1 \text { \& 2, RCFT), Processing } \\
\text { speed (TMT-A), Cognitive } \\
\text { flexibility (TMT-B, WCST, } \\
\text { SCWT), Verbal fluency } \\
\text { (COWAT) }\end{array}$} & \multirow[b]{2}{*}{$\begin{array}{l}\text { OCD: } \downarrow \text { memory, speed } \\
\text { of information processing } \\
\text { and cognitive flexibility. } \\
\text { (After treatment the } \\
\text { cognitive profile of the } \\
\text { OCD group was } \\
\text { normalized) }\end{array}$} \\
\hline & & HC: $13.06 \pm 2.84$ & $\mathrm{HC}(12 \mathrm{M} ; 10 \mathrm{~F})$ & & & \\
\hline & & & & & Clinical: Y-BOCS & \\
\hline
\end{tabular}


Table 3 Neuropsychological studies evaluating the five functional domains in young people (12-30 yrs) with a mood and/or anxiety disorder (Continued)

\begin{tabular}{|c|c|c|c|c|c|}
\hline \multirow[t]{3}{*}{ [53] } & OCD: $29.70 \pm 10.74$ & OCD (12M; 8F) & \multirow{3}{*}{$\begin{array}{l}\text { Examine the impact of } \\
\text { depression on executive } \\
\text { function deficits in OCD }\end{array}$} & \multirow{2}{*}{$\begin{array}{l}\text { NY: VCAT, Verbal Fluency } \\
\text { (COWAT), Processing } \\
\text { speed (TMT-A), Cognitive } \\
\text { flexibility (TMT-B, WCST) }\end{array}$} & \multirow{3}{*}{$\begin{array}{l}\text { OCD: cognitive flexibility } \\
\text { deficits } \sim \text { co-morbid } \\
\text { depression severity }\end{array}$} \\
\hline & \multirow[t]{2}{*}{ HC: $30.06 \pm 10.06$} & \multirow[t]{2}{*}{$\mathrm{HC}(11 \mathrm{M} ; 21 \mathrm{~F})$} & & & \\
\hline & & & & Clinical: MMPI-D & \\
\hline \multirow[t]{4}{*}{ [47] } & $\mathrm{HC}: 12.5 \pm 2.4$ & $\mathrm{HC}(11 \mathrm{M} ; 10 \mathrm{~F})$ & \multirow{4}{*}{$\begin{array}{l}\text { Compare impulsivity at } \\
\text { the neuropsychological } \\
\text { and behavioural level in } \\
\text { young depressed patients } \\
\text { and healthy controls. }\end{array}$} & \multirow{3}{*}{$\begin{array}{l}\text { NU: Cognitive style } \\
\text { (MFFT), Verbal fluency } \\
\text { (VFT), Decision making } \\
\text { (WDWT), cognitive } \\
\text { flexibility (SCWT), } \\
\text { Impulsivity (CPT) }\end{array}$} & \multirow{2}{*}{$\begin{array}{l}\text { DD: } \uparrow \text { symptom severity } \\
\sim \uparrow \text { reaction time, } \downarrow \text { in } \\
\text { commission errors. }\end{array}$} \\
\hline & \multirow[t]{3}{*}{ DD: $11.7 \pm 2.3$} & \multirow[t]{3}{*}{$\mathrm{DD}(11 \mathrm{M} ; 10)$} & & & \\
\hline & & & & & $\begin{array}{l}\text { DD: } \uparrow \text { conservative } \\
\text { response styles \& }\end{array}$ \\
\hline & & & & $\begin{array}{l}\text { Clinical: HDRS, CDI, CPRS- } \\
\text { R:L }\end{array}$ & $\begin{array}{l}\text { reaction times \& } \\
\text { response initiation }\end{array}$ \\
\hline \multirow[t]{4}{*}{ [166] } & HC: $28.2 \pm 7.9$ & $\mathrm{HC}(20 \mathrm{M} ; 28 \mathrm{~F})$ & \multirow{4}{*}{$\begin{array}{l}\text { Investigate the effect of } \\
\text { syndrome state or course } \\
\text { on executive dysfunction }\end{array}$} & \multirow{2}{*}{$\begin{array}{l}\text { NY: Intelligence (NAART), } \\
\text { Cognitive flexibility } \\
\text { (WCST) }\end{array}$} & \multirow{4}{*}{$\begin{array}{l}\text { EUT: } \uparrow \text { cognitive flexibility } \\
\text { than MEM. Performed } \\
\text { similarly to FEM }\end{array}$} \\
\hline & EUT: $30.0 \pm 7.2$ & EUT (11M; 14F) & & & \\
\hline & FEM: $25.7 \pm 9.2$ & FEM (11M; 10F) & & \multirow[t]{2}{*}{ Clinical: YMRS, HDRS } & \\
\hline & MEM: 28.28 .6 & MEM (16M; 18F) & & & \\
\hline \multirow[t]{3}{*}[39]{$^{*}$} & SAD: $23.9 \pm 6.7$ & SAD (20M; 10F) & \multirow{3}{*}{$\begin{array}{l}\text { Assess the } \\
\text { neuropsychological } \\
\text { function of SAD without } \\
\text { co-morbidity }\end{array}$} & \multirow{2}{*}{$\begin{array}{l}\text { NY: Executive function } \\
\text { (CPT, TMT-B, WCST), Pro- } \\
\text { cessing speed (TMT-A), } \\
\text { Verbal learning \& mem- } \\
\text { ory (AVLT) }\end{array}$} & \multirow{3}{*}{$\begin{array}{l}\text { SAD: } \downarrow \text { executive } \\
\text { function } \sim \uparrow \text { SAD severity }\end{array}$} \\
\hline & \multirow[t]{2}{*}{ HC: $25.6 \pm 5.6$} & \multirow[t]{2}{*}{$\mathrm{HC}(20 \mathrm{M} ; 10 \mathrm{~F})$} & & & \\
\hline & & & & Clinical: GAF & \\
\hline \multirow[t]{4}{*}{ [44] } & $\mathrm{HC}: 12.8 \pm 2.5$ & $\mathrm{HC}(15 \mathrm{M} ; 18 \mathrm{~F})$ & \multirow{4}{*}{$\begin{array}{l}\text { Examine basic } \\
\text { performance } \\
\text { neuropsychological } \\
\text { performance in children } \\
\text { and adolescents with } \\
\text { anxiety disorder or } \\
\text { depressive disorder and } \\
\text { in healthy subjects under } \\
\text { drug-free condition }\end{array}$} & \multirow{3}{*}{$\begin{array}{l}\mathrm{N} \Psi \text { : Intelligence (WISC-III), } \\
\text { Verbal learning and } \\
\text { memory (RAVLT), } \\
\text { Attention (go-no go task) }\end{array}$} & \multirow{4}{*}{$\begin{array}{l}\text { DD: } \downarrow \text { verbal learning and } \\
\text { memory compared to } \mathrm{HC} \\
\text { and ANX. }\end{array}$} \\
\hline & ANX: $12.4 \pm 2.3$ & ANX (19M; 15F) & & & \\
\hline & \multirow[t]{2}{*}{ DD: $13.5 \pm 2.6$} & \multirow[t]{2}{*}{ DD (17M; 14F) } & & & \\
\hline & & & & Clinical: CDI & \\
\hline \multirow[t]{2}{*}{ [48] } & \multirow[t]{2}{*}{$\begin{array}{l}\text { HC: } 17.46 \pm 1.59 \\
\text { MDD: } 17.32 \pm 1.59\end{array}$} & \multirow[t]{2}{*}{$\begin{array}{l}\mathrm{HC}(14 \mathrm{M}, 16 \mathrm{~F}) \mathrm{MDD} \\
(12 \mathrm{M}, 19 \mathrm{~F})\end{array}$} & $\begin{array}{l}\text { Investigate whether } \\
\text { major depression in } \\
\text { adolescence is } \\
\text { characterized by } \\
\text { neurocognitive deficits in } \\
\text { attention, affective } \\
\text { decision making, and }\end{array}$ & $\begin{array}{l}\text { NY: Inhibitory control } \\
\text { (CPT, go-no go task), At- } \\
\text { tention (ANT), Decision } \\
\text { making (IGT), Verbal } \\
\text { learning and memory } \\
\text { (RAVLT), Attention (go-no } \\
\text { go task) }\end{array}$ & $\begin{array}{l}\text { MDD: } \uparrow \text { depression } \\
\text { symptom severity } \sim \downarrow \\
\text { cognitive control of } \\
\text { emotion processing }\end{array}$ \\
\hline & & & $\begin{array}{l}\text { cognitive control of } \\
\text { emotion processing }\end{array}$ & Clinical: BDI & \\
\hline$[167]^{*}$ & OCD: $27 \pm 9.8$ & OCD (15M; 16F) & Characterize the cognitive & $\mathrm{N} \Psi$ : cognitive flexibility & Null findings for \\
\hline & HC: $27.4 \pm 9.1$ & $\mathrm{HC}(14 \mathrm{M} ; 16 \mathrm{~F})$ & $\begin{array}{l}\text { with OCD by utilizing } \\
\text { ERPs and } \\
\text { neuropsychological tests }\end{array}$ & $\begin{array}{l}\text { speed (TMT-A), Design } \\
\text { fluency test, Verbal flu- } \\
\text { ency (CWAT) }\end{array}$ & neuropsychological tests. \\
\hline & & & & Clinical: HDRS & \\
\hline [49] & MDD: $15.3 \pm 1.6$ & MDD (5M; 17F) & Investigate verbal fluency, & $\mathrm{N} \Psi:$ Verbal fluency & DD: $\downarrow$ WM \& VF. MDD: \\
\hline & DD: $15.6 \pm 1.5$ & DD (6M; 6F) & speed, and executive & speed (Inspection time & \\
\hline & HC: $15.8 \pm 1.2$ & $\mathrm{HC}(9 \mathrm{M} ; 24 \mathrm{~F})$ & $\begin{array}{l}\text { functions in adolescents } \\
\text { with unipolar depression. }\end{array}$ & $\begin{array}{l}\text { task), Working memory } \\
\text { (Serial choice reaction } \\
\text { time task), Set shifting } \\
\text { (Local-global task) Clinical: }\end{array}$ & \\
\hline$[52]$ & OCD: $24.06 \pm 5$ & OCD (21M; 9F) & Assess the relationship & $\mathrm{N \Psi}$ : Intelligence (WAIS- & Results showed a \\
\hline & HC: Matched & $\mathrm{HC}(21 \mathrm{M} ; 9 \mathrm{~F})$ & dysfunction, clinical status & flexibility (WCST) & recognition, which \\
\hline & & & and severity in OCD. & Clinical: YBOCS & $\begin{array}{l}\text { worsens with chronicity, } \\
\text { deteriorated set-shifting }\end{array}$ \\
\hline
\end{tabular}


Table 3 Neuropsychological studies evaluating the five functional domains in young people (12-30 yrs) with a mood and/or anxiety disorder (Continued)

\begin{tabular}{|c|c|c|c|c|c|}
\hline \multirow[t]{3}{*}{ [55] } & HC: $12.4 \pm 3.3$ & $\mathrm{HC}(15 \mathrm{M} ; 9 \mathrm{~F})$ & \multirow{3}{*}{$\begin{array}{l}\text { Examine the treatment } \\
\text { impact of lamotrigine on } \\
\text { the neurocognitive profile } \\
\text { of patients with pediatric } \\
\text { bipolar disorder }\end{array}$} & \multirow{2}{*}{$\begin{array}{l}\text { NY: Attention (TMT-A, } \\
\text { CPT), cognitive flexibility } \\
\text { (TMT-B), Verbal fluency } \\
\text { (COWT), Working memory } \\
\text { (WMS; DS, SS), Verbal } \\
\text { memory (CVLT) }\end{array}$} & \multirow{3}{*}{$\begin{array}{l}\text { BPD: } \uparrow \text { Working memory } \\
\text { and verbal memory } \\
\text { following treatment (to } \\
\text { levels similar to HC) }\end{array}$} \\
\hline & \multirow[t]{2}{*}{ BPD: $13 \pm 3.1$} & \multirow[t]{2}{*}{$\mathrm{BPD}(18 \mathrm{M} ; 16 \mathrm{~F})$} & & & \\
\hline & & & & Clinical: YMRS & \\
\hline \multirow[t]{3}{*}{ [168] } & MDD: $26.93 \pm 5.33$ & $\operatorname{MDD}(14 \mathrm{M} ; 14 \mathrm{~F})$ & \multirow{3}{*}{$\begin{array}{l}\text { Assess the association } \\
\text { between executive } \\
\text { function and relapse }\end{array}$} & \multirow{2}{*}{$\begin{array}{l}\text { NY: cognitive flexibility } \\
\text { (CWIT, TMT-B), Verbal flu- } \\
\text { ency (VFT), Processing } \\
\text { speed (TMT-A), }\end{array}$} & \multirow{3}{*}{$\begin{array}{l}\text { MDD: Poor BL inhibition } \\
\text { and switching } \sim \uparrow \text { relapse } \\
\text { at FU }\end{array}$} \\
\hline & \multirow[t]{2}{*}{ HC: $26.93 \pm 5.18$} & \multirow[t]{2}{*}{$\mathrm{HC}(14 \mathrm{M} ; 14 \mathrm{~F})$} & & & \\
\hline & & & & Clinical: MADRS & \\
\hline \multirow[t]{2}{*}{ [45] } & \multirow[t]{2}{*}{ CS: $28 \pm 7.9$} & \multirow[t]{2}{*}{ CS (642) } & \multirow{2}{*}{$\begin{array}{l}\text { Examine whether } \\
\text { cognitive deficits predict } \\
\text { current and/or follow-up } \\
\text { (sub)clinical depressive } \\
\text { symptoms in the general } \\
\text { population }\end{array}$} & $\begin{array}{l}\mathrm{N} \Psi \text { : Information } \\
\text { processing (SCWT, CST, } \\
\text { LDST), Episodic memory } \\
(\mathrm{AVLT})\end{array}$ & \multirow[t]{2}{*}{$\begin{array}{l}\text { CS: Poor BL episodic } \\
\text { memory } \sim \text { depressive } \\
\text { symptoms at FUP }\end{array}$} \\
\hline & & & & Clinical: SCL-90 & \\
\hline \multirow[t]{3}{*}{ [169] } & BD: $22.2 \pm 3.9$ & $\mathrm{BD}(23 \mathrm{M} ; 22 \mathrm{~F})$ & \multirow{3}{*}{$\begin{array}{l}\text { Determine whether } \\
\text { neuropsychological } \\
\text { impairments are present } \\
\text { in clinically stable } \\
\text { patients with bipolar } \\
\text { disorder shortly after } \\
\text { resolution of their first } \\
\text { manic episode }\end{array}$} & NY: Intelligence (NAART), & \multirow{3}{*}{$\begin{array}{l}\text { BPD: } \downarrow \text { learning/memory, } \\
\text { spatial/nonverbal } \\
\text { reasoning, executive } \\
\text { function, and some } \\
\text { aspects of attention }\end{array}$} \\
\hline & \multirow[t]{2}{*}{ HC: $22.5 \pm 4.8$} & \multirow[t]{2}{*}{$\mathrm{HC}(12 \mathrm{M} ; 13 \mathrm{~F})$} & & $\begin{array}{l}\text { VISUal spatial reasoning } \\
\text { (K-BIT), Attention/ } \\
\text { processing speed (TMT-A, } \\
\text { CANTAB-RVP, CVLT), } \\
\text { Learning and memory } \\
\text { (CVLT- recall, CANTAB- } \\
\text { SRM;-PRM;-PAL), cognitive } \\
\text { flexibility (TMT-B, } \\
\text { CANTAB-IED;-SWM), Ver- } \\
\text { bal fluency (COWT), }\end{array}$ & \\
\hline & & & & $\begin{array}{l}\text { Clinical: PANSS, HDRS, } \\
\text { BPRS, GAF, YMRS }\end{array}$ & \\
\hline \multirow[t]{2}{*}{ [56] } & \multirow[t]{2}{*}{ MDD: $16.2 \pm 1.1$} & \multirow[t]{2}{*}{ MDD (7M; 11F) } & \multirow[t]{2}{*}{$\begin{array}{l}\text { Investigate the } \\
\text { neurocognitive outcome } \\
\text { in adolescents who were } \\
\text { treated with TMS }\end{array}$} & $\begin{array}{l}\mathrm{N} \Psi \text { : Verbal learning and } \\
\text { memory (CAVLT), } \\
\text { Cognitive flexibility (D- } \\
\text { KEFS, TMT) }\end{array}$ & \multirow[t]{2}{*}{$\begin{array}{l}\text { MDD: } \downarrow \text { Depressive } \\
\text { symptoms were } \\
\text { associated with } \uparrow \text { in } \\
\text { immediate and delayed } \\
\text { verbal memory. }\end{array}$} \\
\hline & & & & Clinical: CDRS-R & \\
\hline
\end{tabular}

abilities, overfocused attention to irrelevant stimuli and delayed selective attention to relevant tasks. Mild cases showed better selective attention than severe cases. Obsessive cases had a defective visual memory, while compulsive cases had delayed perception of task relevant stimuli. Mixed cases showed disturbed information-processing both early and late.

Note. Sample: ANX Anxiety disorder, BPD Bipolar Disorder, BPD-I Bipolar Disorder I, BPD-I-EFD Bipolar Disorder I with Executive Function Deficits, CS Community Sample, DD Depressive disorder, CU Cannabis user, DSH Deliberate Self-Harm, EUT euthymic, FED First-Episode depression, FEM first episode mania, FEP FirstEpisode Psychosis, HC Healthy Controls, HC-B Healthy Control Binge drinker, HC-EFD Healthy Control with Executive Function Deficits, HC-NB Healthy Control Non Binge drinker, MDD Major Depression Disorder, MDD-B Major Depression Disorder Binge drinker, MDD-NB Major Depression Disorder Non Binge drinker, MEM multiple episode mania, MHP Mental Health Patients (mixed diagnosis sample), NSA No Suicide Attempt, NSIB No Suicide Ideation Behaviour, OCD Obsessive Compulsive Disorder, $P C$ Psychiatric Control (i.e. psychiatric diagnosis but no suicide attempt), PSD Psychotic Spectrum Disorder, SA Suicide Attempters, SAD Social Anxiety Disorder, SIB Suicide Ideation Behaviour

Measures: ANT Attention network test, AUDIT Alcohol Use Disorder Identification Test, AVLT Auditory Verbal Learning Test, BDI Beck Depression Inventory, BPRS Brief psychiatric rating scale, BSS Beck Scale for Suicidal Ideation, CANTAB Cambridge Neuropsychological Test Automated Battery [subsets include: FAS Fluency and semantic test, IED Intra/Extra dimensional Set Shift Errors, MS Motor Screening, PAL Paired associates learning, PRM Pattern recognition memory, RVP Rapid Visual Processing hits score, SRM Spatial recognition memory, SSP Spatial span task, SWM Spatial working memory), C-CASA Columbia Classification Algorithm of Suicide Assessment, CDI Children's Depression Inventory, COWAT Controlled Oral Word Association Task, CPRS-R:L Conners Parent Rating Scale- Revised: Long 
Version, CPT Continuous Performance Test, CSHF Colombia Suicide History Form, CST Concept shifting test, CVLT California Verbal Learning Test for Children, D-KEFS Delis-Kaplan Executive Function System, GAF Global Assessment of Functioning, HDRS Hamilton depression rating scale, IGT lowa Gambling Task, K-BIT Kaufman Brief Intelligence Test, LDSB Longest Digit Span Backward, LDSF Longest Digit Span Forward, LDST letter digit substitution test, LM-Ret logical memorypercentage retention, MADRS Montgomery-Asberg Depression Rating Scale, MEPS means-ends problem-solving procedure, MFFT matching familiar figures test, MMPI-D Minnesota Multiphasic Personality Test, depression subscale, NAART North American Adult Reading Test, $N \Psi$ neuropsychological, PANSS positive and negative syndrome scale, PSIS pierce suicide intent scale, RAVLT rey auditory verbal learning test; total score; retention; and/or 20min score, RCF Rey-Osterrieth Complex Figure, SBS Suicide Behavior Screening, SCWT stroop colour and word test, SCL-90 symptom checklist, SCWT stroop colour and word test, SDMT symbol digit modality test, SFS social functioning scale, SHBQ self-harm behavior questionnaire, SIQ suicide ideation questionnaire, SIS suicide intent scale, SOFAS social and occupational functioning assessment scale, = time-line followback, TMT-A trail making test - part A, TMT-B trail making test - part B, VFT verbal fluency task, WAIS-III Wechsler Adult Intelligence Scale [subsets include: S similarities, V Vocabulary, DS Digit symbol, BD Block design, FFD freedom from distractibility], WASI Wechsler abbreviated scale of Intelligence, WISC Wechsler Intelligence Scale For Children, WCST Wisconsin Card Sorting Test, WDWT Walk don't-walk test, WMS Wechsler Memory Scale [subsets include: DS Digit Span, LM logical memory 1 \& 2, SR= story recall, LLT List Learning Test, SS Spatial span, VR Visual Reproduction], WRAT-III wide range achievement test - third edition, WTAR wechsler test of adult reading, Y-BOCS Yale-Brown obsessive-compulsive scale, YMRS young mania rating scale

Findings: $\uparrow=$ Increased, Improved or Higher, $\downarrow=$ Decreased, Reduced or Lower, $\sim=$ 'is associated with', FUP follow-up

*indicates that the study features more than once in the data synthesis

particularly associated with social and economic participation as well as suicide and self-harm behaviours. More specifically, three of the five included studies have shown decision-making and conceptual flexibility impairments to be predictive of suicide and self-harm behaviours [31-33]. Taken together, these studies identify a shared pathophysiology among those who have previously attempted suicide, those who were current suicide attempters and those who were currently self-harming; that is, they all showed characteristic deficits in decision-making and cognitive inflexibility. There is also contrary evidence whereby suicide attempters performed better on the decision making task than depressed patients who hadn't attempted suicide and healthy controls [34], and there were no neuropsychological differences between self-harmers and non-selfharmers [35]. However, these findings may be attributed to methodological differences and/or a modest sample size, especially since the latter study [35] did not distinguish between current self-harmers and previous selfharmers. The notion that impaired decision making and conceptual flexibility may predispose one to suicidal behaviours is supported by evidence showing neurobiological changes in the areas thought to subserve these functions. Namely, structural and functional dysfunction in the orbitofrontal prefrontal cortex have been identified which imply that suicidal behaviour may be associated with deficits in the attribution of importance to stimuli [36-38], although changes in other regions, such as the dorsolateral prefrontal cortex, have been implicated as well [38].

Similar deficits in executive function, particularly in conceptual flexibility, was associated with impaired social and economic participation [39, 40], whereas studies that investigated verbal learning and memory reported conflicting results regarding social and economic participation. Two studies [24, 41] identified a positive relationship with this functional domain, whilst another two studies did not find any association [29, 39]. Notably, logical memory retention (an index of structured learning and memory) was a common measure identified as significant in the positive studies, but it was not utilised in the other two studies; additional studies of structured learning and memory are needed however the evidence to date suggests that there may be an important role for this particular neuropsychological domain with regards to social and economic participation.

\section{Functional domain: clinical syndrome}

The association between cognitive function and the clinical syndromes of mood and anxiety disorders in adolescents and young adults has previously been reviewed extensively (see [42, 43]), and some of the findings of such reviews have been reiterated by the present systematic review (see below). Interestingly, it has been reported that impaired verbal memory is significantly associated with depression (not specified as MDD) [44] as well as the development of depressive symptoms, in a community sample [45]. These findings implicate a dysfunction in memory occurring earlier in the course of depressive syndrome development while, poor executive function may be associated with more persistent MDD [46]. In terms of delineating symptom severity, individuals diagnosed with unipolar depression with higher levels of depressive symptoms also show increased (i.e. delayed) choice reaction time [47], lower cognitive control of emotional processing [48], and processing speed deficits [49] suggesting that a broader range of cognitive (including social) measures are also associated with depression and may also be sensitive to the severity of illness.

Similar deficits in cognition have been observed among those with anxiety disorders. Greater Social Anxiety Disorder (SAD) symptom severity was associated with poor executive function, specifically cognitive inflexibility in SAD patients [39]. Most studies have identified cognitive deficits associated with OCD, such as, impaired verbal and visual memory [50], information processing [51, 52], and cognitive flexibility [51-53]. As observed in unipolar depression, increased symptom severity in those with OCD is associated with worse selective attention [52], however this has also not been uniformly reported [50]. Comorbidity has been identified as another factor related to the cognitive deficits in OCD, whereby comorbid depression was associated 
Table 4 Imaging studies evaluating the five functional domains young people (12-30 yrs) with a mood and/or anxiety disorder

\begin{tabular}{|c|c|c|c|c|c|c|}
\hline $\begin{array}{l}\text { Outcome } \\
\text { measure }\end{array}$ & Study & Age $($ mean $\pm S D)$ & Sample (N) & Aims & Key measures & Key findings \\
\hline \multirow{2}{*}{$\begin{array}{l}\text { Social and } \\
\text { economic } \\
\text { participation }\end{array}$} & \multirow[t]{2}{*}[57]{} & MDD: $15.7 \pm 1.5$ & MDD (8M; 6F) & \multirow{2}{*}{$\begin{array}{l}\text { Assess amygdala activation and } \\
\text { connectivity during an emotional } \\
\text { regulation task. }\end{array}$} & |maging: fMR| & \multirow{2}{*}{$\begin{array}{l}\text { MDD: } \downarrow \text { amygdala-seeded } \\
\text { connectivities } \sim \downarrow \text { social and } \\
\text { economic participation }\end{array}$} \\
\hline & & HC: $15.1 \pm 1.6$ & $\mathrm{HC}(8 \mathrm{M} ; 6 \mathrm{~F})$ & & Functional: CGAS & \\
\hline \multirow[t]{4}{*}{$\begin{array}{l}\text { Physical } \\
\text { health }\end{array}$} & \multirow[t]{4}{*}[58]{} & BPD-O: $23.8 \pm 4.5$ & BPD-O (9M; 11F) & \multirow[t]{4}{*}{$\begin{array}{l}\text { Examine the relationship between BMI } \\
\text { and brain volumes in mania. }\end{array}$} & Imaging: sMRI & $\begin{array}{l}\text { BPD: } \uparrow \mathrm{BMI} \sim \downarrow \text { WMV and } \\
\text { TLV }\end{array}$ \\
\hline & & BPD-N: $22.2 \pm 4.4$ & BPD-N (19M; 18F) & & Functional: BMI & $\mathrm{HC}: \uparrow \mathrm{BMI} \sim \downarrow \mathrm{TBV}$ and GMV. \\
\hline & & HC-O: $22.0 \pm 3.8$ & $\mathrm{HC}-\mathrm{O}(12 \mathrm{M} ; 5 \mathrm{~F})$ & & & \\
\hline & & HC-N: $22.3 \pm 3.5$ & HC-N (19M; 19F) & & & \\
\hline \multirow{16}{*}{$\begin{array}{l}\text { Suicide and } \\
\text { self-harm }\end{array}$} & \multirow[t]{3}{*}{ [170] } & \multirow[t]{3}{*}{ MHP: $14.6 \pm 3.4$} & PSD (18M; 5F) & \multirow{3}{*}{$\begin{array}{l}\text { Compare WMH in psychiatrically } \\
\text { hospitalized youth with and without a } \\
\text { history of suicide attempt }\end{array}$} & Imaging: sMRI & \multirow{3}{*}{$\begin{array}{l}\text { MDD: } \uparrow \text { WMH } \sim \text { suicide } \\
\text { attempt history, but not } \\
\text { ideation }\end{array}$} \\
\hline & & & $\begin{array}{l}\mathrm{BPD}(26 \mathrm{M} ; 9 \mathrm{~F}) \\
\operatorname{MDD}(33 \mathrm{M} ; 15 \mathrm{~F})\end{array}$ & & Functional: PRS & \\
\hline & & & PC (34M; 12F) & & & \\
\hline & \multirow[t]{2}{*}{ [171] } & \multirow[t]{2}{*}{ MDD: $26.7 \pm 5.5$} & \multirow[t]{2}{*}{ MDD (34M; 68F) } & \multirow{2}{*}{$\begin{array}{l}\text { Compare the prevalence and location } \\
\text { of WMH in young MDD inpatients } \\
\text { with and without histories of suicide } \\
\text { attempts }\end{array}$} & |maging: sMR| & \multirow{2}{*}{$\begin{array}{l}\text { MDD: } \uparrow P V H, \text { not DWMH, } ~ \\
\text { suicide attempt history, but } \\
\text { not ideation }\end{array}$} \\
\hline & & & & & $\begin{array}{l}\text { Functional: } \\
\text { Clinical records }\end{array}$ & \\
\hline & \multirow[t]{2}{*}[59]{$^{*}$} & HC: $16.2 \pm 0.8$ & $\mathrm{HC}(4 \mathrm{M} ; 9 \mathrm{~F})$ & \multirow{2}{*}{$\begin{array}{l}\text { Evaluate the ACC volumes of MDD/ } \\
\text { borderline personality patients with } \\
\text { and without a suicide attempt history }\end{array}$} & Imaging: sMRI & \multirow{2}{*}{$\begin{array}{l}\text { MDDx: } \downarrow \text { BA2 } 24 \text { volumes } \sim \uparrow \\
\text { number of suicide attempts } \\
\text { (and } \uparrow \text { borderline severity, } \\
\text { but not depression) }\end{array}$} \\
\hline & & MDDx: $15.8 \pm 1.1$ & MDDx (2M; 11F) & & $\begin{array}{l}\text { Functional: } \\
\text { Clinical interview }\end{array}$ & \\
\hline & \multirow[t]{3}{*}[62]{} & \multirow{3}{*}{$\begin{array}{l}\text { SA: } 16.20 \pm 0.78 \\
\text { PC: } 15.87 \pm 1.55 \\
\text { HC: } 15.21 \pm 1.42\end{array}$} & $\mathrm{SA}(4 \mathrm{M} ; 11 \mathrm{~F})$ & \multirow{3}{*}{$\begin{array}{l}\text { Evaluate the association between } \\
\text { neural activity during performance of } \\
\text { the go no-go task and suicide history. }\end{array}$} & Imaging: fMRI & \multirow{3}{*}{$\begin{array}{l}P C: \uparrow \text { activity in right } A C G \\
\text { compared to } S A \text { (but } S A \text { not } \\
\text { different from } \mathrm{HC} \text { ) }\end{array}$} \\
\hline & & & $\mathrm{PC}(7 \mathrm{M} ; 8 \mathrm{~F})$ & & Functional: CSHF & \\
\hline & & & $\mathrm{HC}(8 \mathrm{M} ; 6 \mathrm{~F})$ & & & \\
\hline & \multirow[t]{3}{*}[60]{} & SA: $16.21 \pm 0.80$ & $\mathrm{SA}(4 \mathrm{M} ; 10)$ & \multirow{3}{*}{$\begin{array}{l}\text { Measure neural activity during } \\
\text { processing of emotional faces in } \\
\text { adolescents with a history of } \\
\text { depression and suicide attempt }\end{array}$} & Imaging: fMRI & \multirow{3}{*}{$\begin{array}{l}\text { SA: } \uparrow \text { dorsal ACG activity } \\
\text { when viewing angry faces, } \\
\text { and } \downarrow \text { visual, sensory, } \\
\text { prefrontal, ACG activity to } \\
\text { intense happy and neutral } \\
\text { faces } \sim \text { suicide attempt } \\
\text { history. }\end{array}$} \\
\hline & & PC: $15.87 \pm 1.55$ & $\mathrm{PC}(7 \mathrm{M} ; 8 \mathrm{~F})$ & & Clinical: C-CASA, & \\
\hline & & $H C: 15.27 \pm 1.39$ & $\mathrm{HC}(8 \mathrm{M} ; 7 \mathrm{~F})$ & & CSHF, SIQ, SIS & \\
\hline & \multirow[t]{3}{*}[34]{$^{*}$} & SA: $16.20 \pm 0.78$ & $\mathrm{SA}(4 \mathrm{M} ; 11 \mathrm{~F})$ & \multirow{3}{*}{$\begin{array}{l}\text { Measure neural activity during } \\
\text { performance on the IGT in } \\
\text { adolescents. }\end{array}$} & Imaging: fMRI & PC: $\uparrow$ hippocampal activity \\
\hline & & $P C: 15.79 \pm 1.58$ & PC (7M; 7F) & & Functional: C- & $\begin{array}{l}\text { compared to } \mathrm{HC} \text {. (HC and } \\
\text { SA did not differ, evidence }\end{array}$ \\
\hline & & HC: $15.15 \pm 1.46$ & $\mathrm{HC}(8 \mathrm{M} ; 5 \mathrm{~F})$ & & $\begin{array}{l}\text { CASA, CSHF, SIQ, } \\
\text { SIS }\end{array}$ & of $\downarrow$ activation) \\
\hline Alcohol and & {$[172]$} & BPD-L: $23.7 \pm 3.6$ & BPD-L (14M; 5F) & Assess the effects of alcohol use on & Imaging: MRS & BPD-H: $\downarrow$ GSH \\
\hline & & BPD-H: $23.4 \pm 3.1$ & $\mathrm{BPD}-\mathrm{H}(12 \mathrm{M} ; 2 \mathrm{~F})$ & & Functional: & \\
\hline & & HC: $23.6 \pm 2.8$ & $\mathrm{HC}(13 \mathrm{M} ; 4 \mathrm{~F})$ & & & \\
\hline & [65] & MDD: $21.7 \pm 2.0$ & $\operatorname{MDD}(5 \mathrm{M} ; 1 \mathrm{~F})$ & Examine the effect of cannabis use on & Imaging: fMRI & MDD: $\uparrow C U \sim \downarrow$ amygdala \\
\hline & & & & & $\begin{array}{l}\text { Functional: SCID } \\
\text { (presence of } \\
\text { dependence) }\end{array}$ & \\
\hline & {$[66]$} & AUD: $17 \pm 2.1$ & AUD $(8 M ; 6 F)$ & Compare prefrontal-thalamic- & Imaging: sMRI & AUD: $\downarrow$ PFC \& PFC WMV. \\
\hline & & HC: $16.9 \pm 2.3$ & $\mathrm{HC}(16 \mathrm{M} ; 12 \mathrm{~F})$ & $\begin{array}{l}\text { and young adults with adolescent- } \\
\text { onset alcohol use disorders }\end{array}$ & $\begin{array}{l}\text { Fucntional: ACQ, } \\
\text { LHAUI, SCID }\end{array}$ & $\begin{array}{l}\text { AUD: } \downarrow \text { PFC GM } \sim \uparrow \text { alcohol } \\
\text { consumption }\end{array}$ \\
\hline & & & & & & $A \cup D(M): \downarrow C V$ \\
\hline & & & & & & $\begin{array}{l}\text { PFC volume variables } \\
\text { measures of alcohol } \\
\text { consumption }\end{array}$ \\
\hline & [64] & BPD: $16 \pm 2$ & $\mathrm{BPD}(5 \mathrm{M} ; 9 \mathrm{~F})$ & Compare brain morphometry in & Imaging: sMRI & BPD: CUD $\sim \downarrow$ LFG GMV \& $\uparrow$ \\
\hline & & & & substance and alcohol disorders & $\begin{array}{l}\text { Functional: ASI, } \\
\text { SCID, SAC }\end{array}$ & \\
\hline
\end{tabular}


Table 4 Imaging studies evaluating the five functional domains young people (12-30 yrs) with a mood and/or anxiety disorder (Continued)

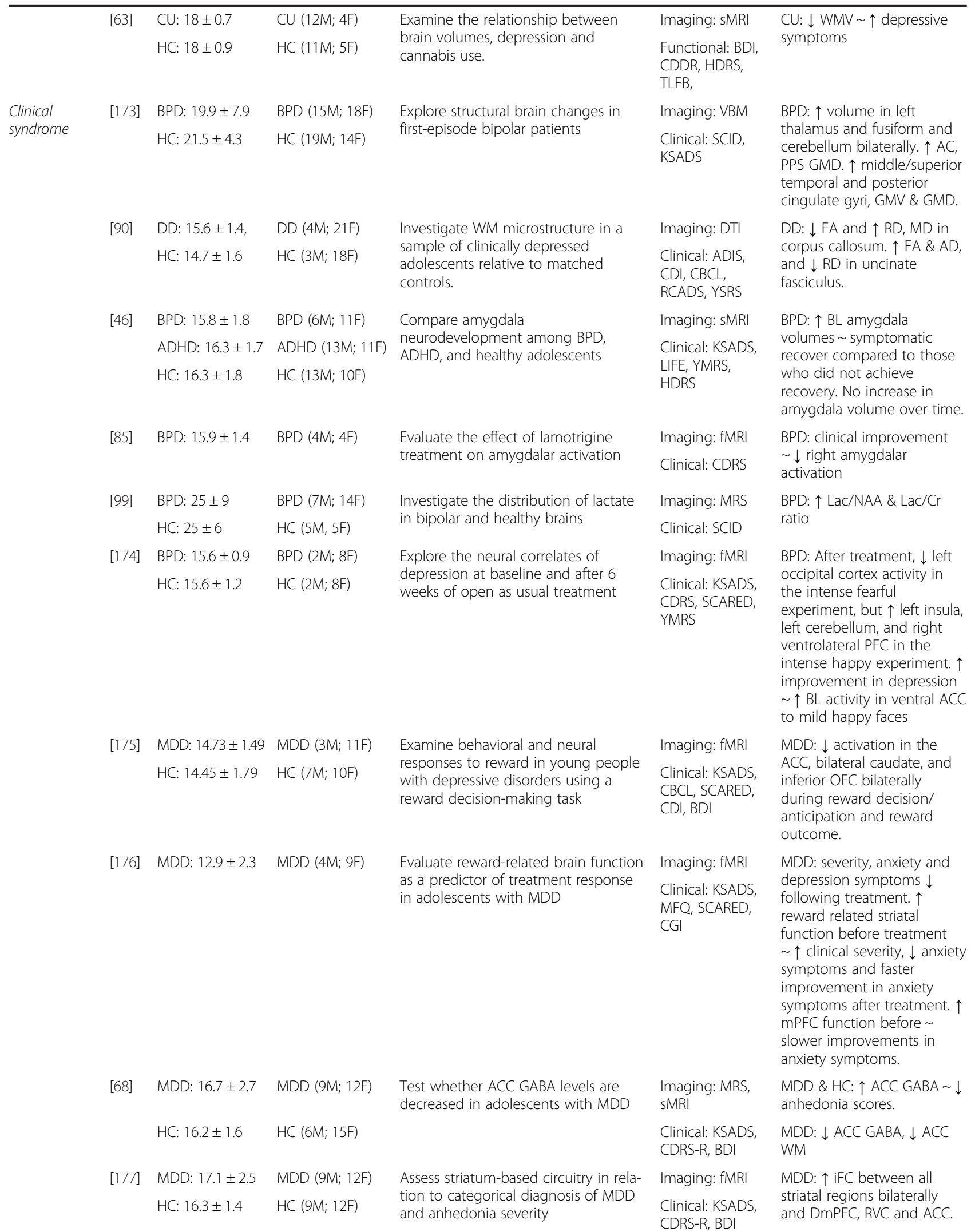


Table 4 Imaging studies evaluating the five functional domains young people (12-30 yrs) with a mood and/or anxiety disorder (Continued)

MDD severity iFC between the striatum and the precuneus, posterior cingulate cortex and dmPFC. Anhedonia severity Pregenual ACC, subgenual ACC, supplementary motor area, and supramarginal gyrus iFC.

$\begin{array}{lll}\text { [178] } & \text { BPD: } 15.1 \pm 1.81 & \text { BPD (6M; 12F) } \\ & \text { HC: } 14.1 \pm 1.61 & \text { HC (6M; 12F) } \\ & & \\ \text { [91] } & \text { OCD: } 12.35 \pm 2.93 & \text { OCD (7M; 14F) } \\ & \text { HC: } 12.47 \pm 8.33 & \text { HC (7M; 14F) }\end{array}$

[179] OCD: $13.1 \pm 2.5 \quad$ OCD (11M; 7F)

$H C: 13.6 \pm 2.4 \quad H C(11 M ; 7 F)$
Investigate the brain structural changes in BPD children and adolescents

Measure neuroanatomical changes in the thalamus of patients with OCD near the onset of illness, and before and after treatment.
Imaging: DTI

Clinical: KSADS, YMRS, MFQ

Imaging: MRI

Clinical: KSADS, YBOCS, HDRS

Examine whether overlapping but symptom dimension-specific neural activity patterns in adults are apparent in youths
Imaging: $\mathrm{fMRI}$

Clinical: YBOCS

\section{BPD: $\downarrow$ GMV in left} hippocampus. $\downarrow$ FA value in rACC.

$\downarrow$ hippocampal volume $\sim \uparrow$ YMRS score

OCD: $\uparrow$ thalamic volumes in treatment naïve patients. $\downarrow$ thalamic volumes (to comparable levels with controls) paroxetine monotherapy.

$\downarrow$ thalamic volumes $\sim$ OCD symptom severity

OCD: $\downarrow$ activity in right insula, putamen, thalamus, dorsolateral prefrontal cortex and left orbitofrontal cortex, and right thalamus and right insula. $\uparrow$ OCD symptom related measures were significantly predictive of $\downarrow$ neural activity in the right dorsolateral prefrontal cortex during the contamination experiment.

\begin{tabular}{|c|c|c|c|}
\hline \multirow[t]{2}{*}[59]{$^{*}$} & $\mathrm{HC}: 16.2 \pm 0.8$ & $\mathrm{HC}(4 \mathrm{M} ; 9 \mathrm{~F})$ & \multirow{2}{*}{$\begin{array}{l}\text { Evaluate the ACC volumes of MDD/ } \\
\text { borderline personality patients with } \\
\text { and without a suicide attempt history }\end{array}$} \\
\hline & MDDx: $15.8 \pm 1.1$ & MDDx (2M; 11F) & \\
\hline \multirow[t]{2}{*}{ [94] } & OCD: $14.3 \pm 2.1$ & OCD (13M; 10F) & \multirow{2}{*}{$\begin{array}{l}\text { Investigate white matter abnormalities } \\
\text { in pediatric obsessive-compulsive } \\
\text { disorder. }\end{array}$} \\
\hline & HC: $14.2 \pm 2.2$ & $\mathrm{HC}(12 \mathrm{M} ; 11 \mathrm{~F})$ & \\
\hline \multirow[t]{2}{*}{ [180] } & MHP: $22.3 \pm 3.7$ & $\mathrm{MHP}(50 \mathrm{M} ; 83 \mathrm{~F})$ & \multirow{2}{*}{$\begin{array}{l}\text { Examine the relationship between } \\
\text { anterior insula GMV, clinical symptom } \\
\text { severity and neuropsychological } \\
\text { performance. }\end{array}$} \\
\hline & HC: $23.8 \pm 2.4$ & $\mathrm{HC}(13 \mathrm{M} ; 26 \mathrm{~F})$ & \\
\hline \multirow[t]{2}{*}{ [95] } & MDD: $16.8 \pm 2.2$ & MDD (9M; 8F) & \multirow{2}{*}{$\begin{array}{l}\text { Investigate WM microstructure } \\
\text { in MDD using diffusion tensor imaging }\end{array}$} \\
\hline & HC: $16.4 \pm 1.4$ & $\mathrm{HC}(6 \mathrm{M} ; 10 \mathrm{~F})$ & \\
\hline \multirow[t]{2}{*}{ [181] } & MDD: $15.8 \pm 1.4$ & $\operatorname{MDD}(8 \mathrm{M} ; 11 \mathrm{~F})$ & \multirow{2}{*}{$\begin{array}{l}\text { Investigate sgACC FC in adolescent } \\
\text { depression during negative emotional } \\
\text { processing. }\end{array}$} \\
\hline & $H C: 16.1 \pm 1.2$ & $\mathrm{HC}(8 \mathrm{M} ; 11 \mathrm{~F})$ & \\
\hline
\end{tabular}

Imaging: SMRI

Clinical: Clinical interview

Imaging: DTI

Clinical: YBOCS, KSADS

Imaging: SMRI

Clinical: BPRS, HDRS, SOFAS

Imaging: DTI

Clinical: KSADS, BDI, CDRS-R, MASC

MDDx: $\downarrow$ BA24 volumes $\sim \uparrow$ borderline severity, but not depression

ODD: $\uparrow \mathrm{FA}$ in splenium $\sim \uparrow$ obsession severity

MHP: $\downarrow$ GMV in left anterior insula. Changes ( $\uparrow$ or $\downarrow$ ) in right anterior insula GMV $\sim \uparrow$ symptom severity.

MDD: $\downarrow$ WM integrity in the genu of corpus callosum, anterior thalamic radiation, anterior cingulum and sagittal stratum $\sim \uparrow$ depression severity.

Imaging: $\mathrm{FMR}$ Clinical: BDI

MDD: $\uparrow$ sgACC- amygdala Functional connectivity and $\downarrow$ sgACC-fusiform gyrus, sgACC-precuneus, sgACCinsula, and sgACC-middle frontal gyrus functional connectivity. $\downarrow$ sgACC-precuneus functional connectivity $\sim \uparrow$ depression severity.

[93] $H C: 16 \pm 2.74 \quad H C(6 M ; 7 F)$
Imaging: DTI
Evaluate whether the observed WM disruptions are associated with
MDD at FUP $\sim$ FA in the superior longitudinal fasciculi 
Table 4 Imaging studies evaluating the five functional domains young people (12-30 yrs) with a mood and/or anxiety disorder (Continued)

\begin{tabular}{|c|c|c|c|c|c|}
\hline & MT: $15.89 \pm 2.79$ & MT (5M; 14F) & $\begin{array}{l}\text { increased vulnerability to } \\
\text { psychopathology during prospective } \\
\text { follow-up }\end{array}$ & $\begin{array}{l}\text { Clinical: KSADS, } \\
\text { FH-RDC, CDRS-R, } \\
\text { HDRS, CGAS, BDI } \\
\text { DUSI }\end{array}$ & $\begin{array}{l}\text { \& the right cingulum- } \\
\text { hippocampal Projection. } \\
\text { SUD at FUP } \sim \downarrow \text { FA in the } \\
\text { right cingulum-hippocampal } \\
\text { projection. }\end{array}$ \\
\hline \multirow[t]{2}{*}{ [182] } & OCD: $13.95 \pm 2.52$ & OCD (9M; 16F) & \multirow{2}{*}{$\begin{array}{l}\text { Investigate the development of the } \\
\text { ACC and its associations with } \\
\text { psychopathology. }\end{array}$} & Imaging: fMRI & \multirow{2}{*}{$\begin{array}{l}\text { OCD: } \uparrow \text { ACC activity during } \\
\text { error responses in bilateral } \\
\text { insular cortex during high } \\
\text { conflict tasks }\end{array}$} \\
\hline & HC: $13.71 \pm 2.85$ & $\mathrm{HC}(9 \mathrm{M} ; 16 \mathrm{~F})$ & & $\begin{array}{l}\text { Clinical: YBOCS, } \\
\text { ADIS, CDI, STAI- } \\
\text { C, CBCL }\end{array}$ & \\
\hline \multirow[t]{2}{*}{ [81] } & OCD: $13.78 \pm 2.58$ & OCD (11M; 18F) & \multirow{2}{*}{$\begin{array}{l}\text { Identify differences in regional brain } \\
\text { volume between medication-free } \\
\text { pediatric OCD patients and controls } \\
\text { and examine changes after cognitive } \\
\text { behavioural therapy }\end{array}$} & Imaging: VBM & \multirow{2}{*}{$\begin{array}{l}\text { OCD: } \uparrow \text { Orbitofrontal GMV } \\
\text { after treatment } \sim \uparrow \text { symptom } \\
\text { improvement }\end{array}$} \\
\hline & $H C: 13.6 \pm 2.73$ & $\mathrm{HC}(11 \mathrm{M} ; 18 \mathrm{~F})$ & & $\begin{array}{l}\text { Clinical: YBOCS, } \\
\text { ADIS, CDI, STAI- } \\
\text { C, CBCL }\end{array}$ & \\
\hline \multirow[t]{3}{*}{ [183] } & BPD-I: $14.57 \pm 1.98$ & BPD-I (11M; 7F) & \multirow{3}{*}{$\begin{array}{l}\text { Examine patterns of activity and } \\
\text { connectivity in youth with BPD. }\end{array}$} & Imaging: fMRI & \multirow{3}{*}{$\begin{array}{l}\text { BP-I: } \uparrow \text { activity in amygdala } \\
\text { and VMPFC regulation } \\
\text { regions to happy faces and } \\
\text { reduced DLPFC activity to } \\
\text { fearful faces compared to } \\
\text { HC. BPD-NOS: } \downarrow \text { PFC activity } \\
\text { to neural faces compared to } \\
\text { HC. }\end{array}$} \\
\hline & $\begin{array}{l}\text { BPD-NOS: } 12.59 \\
\pm 2.27\end{array}$ & BPD-NOS (11M; 7F) & & \multirow{2}{*}{$\begin{array}{l}\text { Clinical: KSADS, } \\
\text { MFQ, SCARED, } \\
\text { CALS }\end{array}$} & \\
\hline & HC: $13.67 \pm 2.55$ & $\mathrm{HC}(7 \mathrm{M} ; 11 \mathrm{~F})$ & & & \\
\hline \multirow[t]{3}{*}{ [71] } & HC: $23.9 \pm 2.3$ & $\mathrm{HC}(12 \mathrm{M} ; 21 \mathrm{~F})$ & \multirow{3}{*}{$\begin{array}{l}\text { Evaluate patterns of grey matter } \\
\text { changes very early in the course of } \\
\text { affective illness compared to those } \\
\text { with discrete disorders and/or illness } \\
\text { persistence }\end{array}$} & Imaging: sMRI & \multirow{3}{*}{$\begin{array}{l}\mathrm{ST}-2 / 3: \downarrow \mathrm{GMV} \text { in frontal } \\
\text { brain regions }\end{array}$} \\
\hline & ST-1: $20.4 \pm 5.2$ & ST-1 (8M; 15F) & & Clinical: HDRS, & \\
\hline & ST-2/3: $23.5 \pm 3.5$ & ST-2/3 (14M; 10F) & & SOFAS, BPRS & \\
\hline \multirow[t]{3}{*}{ [96] } & HC: $23.82 \pm 2.52$ & $\mathrm{HC}(15 \mathrm{M} ; 24 \mathrm{~F})$ & \multirow{3}{*}{$\begin{array}{l}\text { Examine the association between } \\
\text { microstructural WM changes and } \\
\text { different stages of psychiatric illness. }\end{array}$} & Imaging: DTI & \multirow{2}{*}{$\begin{array}{l}\text { ST-2/3: } \downarrow \text { FA within the left } \\
\text { anterior corona radiata } \\
\text { compared to HC. }\end{array}$} \\
\hline & ST-1B: $21.36 \pm 3.51$ & ST-1B (24M; 49F) & & $\begin{array}{l}\text { Clinical: HDRS, } \\
\text { BPRS, SOFAS }\end{array}$ & \\
\hline & ST-2/3: $22.45 \pm 4.35$ & ST-2/3 (37M; 32) & & & $\begin{array}{l}\text { ST-1B: pattern of } \downarrow \text { FA within } \\
\text { the left anterior corona } \\
\text { radiate (less WM } \\
\text { involvement than ST-2/3) }\end{array}$ \\
\hline \multirow[t]{2}{*}{ [92] } & BPD: $23.03 \pm 5.04$ & $\mathrm{BPD}(23 \mathrm{M} ; 35 \mathrm{~F})$ & \multirow{2}{*}{$\begin{array}{l}\text { Examine WM microstructural changes } \\
\text { in BPD. }\end{array}$} & Imaging: DTI & \multirow{2}{*}{$\begin{array}{l}\text { BPD: } \downarrow \text { FA in the genu, body } \\
\text { and splenium of the corpus } \\
\text { callosum as well as the } \\
\text { superior and anterior corona } \\
\text { radiata. } \uparrow \text { radial diffusivity. }\end{array}$} \\
\hline & $H C: 24.05 \pm 2.92$ & $\mathrm{HC}(12 \mathrm{M} ; 28 \mathrm{~F})$ & & $\begin{array}{l}\text { Clinical: HDRS, } \\
\text { YMRS, SOFAS, } \\
\text { BPRS }\end{array}$ & \\
\hline \multirow[t]{2}{*}{ [184] } & OCD: $13.1 \pm 2.7$ & OCD (7M; 5F) & \multirow{2}{*}{$\begin{array}{l}\text { Investigate possible regional brain } \\
\text { dysfunction in premotor cortico- } \\
\text { striatal activity, correlate brain activa- } \\
\text { tion with severity of obsessive- } \\
\text { compulsive symptomatology; And, de- } \\
\text { tect possible changes in brain activity } \\
\text { after pharmacological treatment }\end{array}$} & Imaging: fMRI & \multirow{2}{*}{$\begin{array}{l}\text { OCD: } \uparrow \text { activation bilaterally } \\
\text { in the middle frontal gyrus. } \\
\text { Clinical improvement } \\
\text { following pharmacological } \\
\text { treatment } \sim \downarrow \text { activation in } \\
\text { left insula and left putamen }\end{array}$} \\
\hline & HC: $13.7 \pm 2.8$ & $\mathrm{HC}(7 \mathrm{M} ; 5 \mathrm{~F})$ & & $\begin{array}{l}\text { Clinical: ChIPS, Y- } \\
\text { BOCS, CDI, STAI- } \\
\text { C, LOI-CV }\end{array}$ & \\
\hline \multirow[t]{2}{*}{ [98] } & OCD: $12.5 \pm 2.9$ & OCD (6M; 5F) & \multirow{2}{*}{$\begin{array}{l}\text { Measure neurometabolite } \\
\text { concentrations in anterior cingulate- } \\
\text { medial frontal cortex and right and left } \\
\text { striatum of drug naïve children and } \\
\text { adolescents with OCD }\end{array}$} & Imaging: MRS & \multirow{2}{*}{$\begin{array}{l}\text { OCD: } \downarrow \text { total Cho in left } \\
\text { striatum (this } \downarrow \text { did not } \\
\text { change over time and } \\
\text { persisted at follow-up } \\
\text { Assessment) }\end{array}$} \\
\hline & HC: $14.5 \pm 2.8$ & $\mathrm{HC}(5 \mathrm{M} ; 7 \mathrm{~F})$ & & $\begin{array}{l}\text { Clinical: Y-BOCS, } \\
\text { CDI, STAI-C, LOI- } \\
\text { CV }\end{array}$ & \\
\hline \multirow[t]{2}{*}{ [185] } & BPD: $27 \pm 10$ & $\mathrm{BPD}(26 \mathrm{M} ; 32 \mathrm{~F})$ & \multirow[t]{2}{*}{ Assess changes in GMV in BPD. } & Imaging: sMRI & \multirow[b]{2}{*}{$\begin{array}{l}\text { BPD: } \uparrow \text { GMV in portions of } \\
\text { the VLPFC and hippocamps } \\
\text { complex. } \uparrow \text { GMV in } \\
\text { amygdala proper and } \\
\text { caudate. } \uparrow \text { number of } \\
\text { depressive episodes } \sim \uparrow \text { GMV } \\
\text { in the right cingulate gyrus } \\
\text { bilaterally and right } \\
\text { thalamus and bilateral } \\
\text { lenticulate nuclei, and left } \\
\text { cerebellar vermis. } \uparrow \text { illness }\end{array}$} \\
\hline & $\mathrm{HC} 27 \pm 10$ & $\mathrm{HC}(21 \mathrm{M} ; 27 \mathrm{~F})$ & & $\begin{array}{l}\text { Clinical: SCID, } \\
\text { KSADS }\end{array}$ & \\
\hline
\end{tabular}


Table 4 Imaging studies evaluating the five functional domains young people (12-30 yrs) with a mood and/or anxiety disorder (Continued)

\begin{tabular}{|c|c|c|}
\hline \multirow[t]{2}{*}{ [83] } & OCD: $12.79 \pm 2.64$ & $\mathrm{OCD}(10 \mathrm{M} ; 21 \mathrm{~F})$ \\
\hline & $H C: 12.89 \pm 2.66$ & $\mathrm{HC}(10 \mathrm{M} ; 21 \mathrm{~F})$ \\
\hline \multirow[t]{2}{*}{ [74] } & MDD: 8 - 17years & $\operatorname{MDD}(10 \mathrm{M} ; 13 \mathrm{~F})$ \\
\hline & HC: 8-17 years & $\mathrm{HC}(10 \mathrm{M} ; 13 \mathrm{~F})$ \\
\hline [87] & ANX: $11.8 \pm 1.8$ & ANX $(6 \mathrm{M} ; 6 \mathrm{~F})$ \\
\hline \multirow[t]{2}{*}{ [78] } & SAD: $21.80 \pm 3.68$ & SAD (14M; 6F) \\
\hline & HC: $21.58 \pm 3.72$ & $\mathrm{HC}(13 \mathrm{M} ; 6 \mathrm{~F})$ \\
\hline \multirow[t]{2}{*}{ [69] } & DD: $15.4 \pm 1.5$ & $\mathrm{DD}(3 \mathrm{M} ; 23 \mathrm{~F})$ \\
\hline & HC: $14.7 \pm 1.5$ & $\mathrm{HC}(3 \mathrm{M} ; 23 \mathrm{~F})$ \\
\hline \multirow[t]{2}{*}{ [100] } & BPD: $15.5 \pm 1.5$ & $\mathrm{BPD}(5 \mathrm{M} ; 23 \mathrm{~F})$ \\
\hline & HC: $14.6 \pm 1.8$ & $\mathrm{HC}(4 \mathrm{M} ; 6 \mathrm{~F})$ \\
\hline \multirow[t]{2}{*}{ [186] } & BPD: $14.3 \pm 1.1$ & $\mathrm{BPD}(6 \mathrm{M} ; 11 \mathrm{~F})$ \\
\hline & HC: $14.1 \pm 2.4$ & $\mathrm{HC}(7 \mathrm{M} ; 7 \mathrm{~F})$ \\
\hline
\end{tabular}
[187] BPD:
HC: $13.9 \pm 3.4 \quad H C(7 M ; 7 F)$

Examine the relationships between pretreatment amygdala activity and treatment response in a sample of anxious children and adolescents

Explore the GMD deficits in drug-naïve Imaging: VBM adult SAD patients

Examine GMV in brain areas putatively involved in affective psychopathology.

Compare in vivo neurometabolite concentrations in bipolar adolescents with a depressed episode

Investigate the effects of pharmacotherapy on brain function underlying affect dysregulation and cognitive function in pediatric bipolar disorder.

Determine the relative effects of risperidone and divalproex on brain function in pediatric mania

duration $\sim$ GMV in left cerebellar vermis.

Imaging: fMRI

Clinical: KSADS, CGI
Imaging: MRI

Clinical: YBOCS, HAMA, HDRS

Imaging: MRI

Clinical: CDRS-R, HAMA

Clinical: HAMA HDRS, LSAS, SCID

Imaging: VBM

Clinical: ADIS, CDI, RCADS, YSR, $\mathrm{CBCL}$

Imaging: MRS

Clinical: KSADS,

CDRS-R

Imaging: fMRI

Clinical: YMRS,

KSADS, CDI,

CDRS-R

Imaging: fMR|

Clinical: KSADS, CDRS, YMRS

OCD: $\downarrow$ pituitary gland volume $\sim \uparrow$ compulsive symptom severity (more pronounced in males).

MDD: $\uparrow$ left and right amygdala: hippocampus volume ratios $\sim \uparrow$ severity of anxiety (but not $\uparrow$ depression severity or duration of illness)

ANX: $\uparrow$ left amygdala activation pre-treatment treatment response to CBT or medication. (no association between pre-treatment symptom severity and pretreatment amygdala activity)

SAD: $\downarrow$ GMD in bilateral thalami, right amygdala, and right precuneus. $\downarrow$ right amygdala GMD $\uparrow$ disease duration and $\downarrow$ age of onset.

DD: $\downarrow$ bilateral dorsal ACC volume. No association with clinical severity of depression or anxiety.

BPD: $\uparrow$ NAA in the ACC and VLPFC. $\uparrow$ Cho and $\mathrm{Cr}$ in the VLPFC.

BPD: YMRS improvement $\sim \downarrow$ VMPFC activity.

Normalization of activity in the inferior frontal gyrus following pharmacological treatment.

BPD: Divalproex treatment $\sim \uparrow$ activity in left MPFC relative and modulation of positive emotions to risperidone. $\uparrow$ pre-treatment right amygdala activity with negative and positive condition in the risperidone group, and left amygdala with positive condition in divalproex group predicted poor response on YMRS.

Examine the change in amygdalainsula-medial frontal function during perception of social threat cues before and after SSRI treatment
Imaging: $\mathrm{fMR}$

Clinical: SCID LSAS, HDRS, BDI, STAI
Identify neuroimaging predictors of medication response in contamination-related obsessive compulsive disorder OCD
Imaging: PET

Clinical: AAS, OCDAS
gSP: SSRI treatment $\sim \downarrow$ amygdala reactivity to fearful faces (which was $\uparrow$ pretreatment) and $\uparrow$ ventral MPF activity to angry faces (which was $\downarrow$ Pre-treatment treatment). No correlations with symptom improvement.

OCD: $\downarrow$ rCBF in OFC and $\uparrow$ rCBF values in PCC predicted better fluvoxamine treatment response. 
Table 4 Imaging studies evaluating the five functional domains young people (12-30 yrs) with a mood and/or anxiety disorder (Continued)

\begin{tabular}{|c|c|c|c|c|}
\hline \multirow[t]{2}{*}{ [70] } & HC: $17.19 \pm 1.87$ & $\mathrm{HC}(7 \mathrm{M} ; 9 \mathrm{~F})$ & \multirow{2}{*}{$\begin{array}{l}\text { Investigate the role of dysregulation of } \\
\text { frontal-limbic circuits in the sympto- } \\
\text { mology of this disorder }\end{array}$} & Imaging: sMRI \\
\hline & MDD: $16.89 \pm 2.01$ & MDD (9M; 21F) & & $\begin{array}{l}\text { Clinical: CDRS, } \\
\text { KSADS, BDI }\end{array}$ \\
\hline \multirow[t]{2}{*}{ [84] } & OCD: $12.70 \pm 3.11$ & $\mathrm{OCD}(13 \mathrm{M} ; 8 \mathrm{~F})$ & \multirow{2}{*}{$\begin{array}{l}\text { Investigate the regional morphology } \\
\text { of the CC in OCD. }\end{array}$} & Imaging: sMRI \\
\hline & HC: $12.74 \pm 3.12$ & $\mathrm{HC}(13 \mathrm{M} ; 8 \mathrm{~F})$ & & $\begin{array}{l}\text { Clinical: YBOCS, } \\
\text { HAMA, HDRS, } \\
\text { KSADS }\end{array}$ \\
\hline
\end{tabular}

[79] OCD: $12.89 \pm 3.23 \quad \mathrm{OCD}(5 \mathrm{M} ; 6 \mathrm{~F})$

[73] MDD: $15.35 \pm .34, \operatorname{MDD}(3 \mathrm{M} ; 17 \mathrm{~F})$

HC: $14.08 \pm .31 \quad$ HC $(8 \mathrm{M} ; 16 \mathrm{~F})$

[75] GAD: $22.9 \pm 4.1, \quad$ GAD (16F)

HC: $23.7 \pm 3.7 \quad H C(15 F)$

[189] BPD: $14.6 \pm 2.2 \quad \mathrm{BPD}(11 \mathrm{M} ; 12 \mathrm{~F})$

HC: $15.0 \pm 1.8 \quad H C(6 \mathrm{M} ; 4 \mathrm{~F})$

[101] BPD-R: $15.4 \pm 1 \quad$ BPD-R (4M; 3F)

BPD-NR: $14.1 \pm 2.2$ BPD-NR (6M; 1F)

HC: $14.4 \pm 1.6 \quad$ HC (6M; 9F)

[88] BPD-RE: $13.5 \pm 2.4 \quad$ BPD-RE (13M; 9F) BPD-NRE:

$13.3 \pm 2.0$

$H C: 14.2 \pm 3.1 \quad H C(7 M ; 7 F)$

[72] MDD: $28.8 \pm 10.7$ MDD (35M; 30F)

HC: $28.4 \pm 10.7 \quad H C(37 M ; 56 F)$

[82] OCD: $16.6 \pm 1.5 \quad \operatorname{OCD}(14 \mathrm{M} ; 12 \mathrm{~F})$

$H C: 16.5 \pm 1.4 \quad H C(14 M ; 12 F)$

[80] OCD: $22.0 \pm 5.2 \quad \mathrm{OCD}(3 \mathrm{M} ; 5 \mathrm{~F})$

HC: $21.5 \pm 5.9 \quad H C(8 F)$
Evaluate neuroanatomic changes in the thalamus of OCD patients near illness onset before and after cognitive behavioral therapy

Examine amygdala and hippocampus volumes in pediatric MDD.

Investigate the neural substrates associated with excessive and persistent worrying in GAD

Examine the neurofunctional effects of Imaging: fMRI ziprasidone in manic adolescents

Evaluate the in vivo effects of extended-release divalproex sodium on the glutamatergic system in adolescents with BPD and neurochemical predictors of clinical remission.

Determine functional connectivity among patients with pediatric BPD who are responders to pharmacotherapy and those who are nonresponders,

Evaluate the early effects of antidepressant therapy, as well as of key clinical variables, on ACC volume microstructure changes in pediatric OCD

Evaluated resting brain metabolism and treatment response in $O C D$ patients.

Imaging: SMRI

Clinical: YBOCS,

HDRS, HAMA, KSADS

Imaging: SMRI

Clinical: HDRS,

Imaging: SMRI

Clinical: SCID, $\mathrm{BDI}, \mathrm{MCQ}$

Clinical: YMRS, CGI, KSADS

Imaging: MRS

Clinical: YMRS,

Imaging: SMRI, DTI

Imaging: PET, MRI
Identify structural GM and WM FH-RDC, KSADS

Clinical: KSADS, CDRS, CGI, YMRS

MDD: $\uparrow$ right and left rostral MFG, and left caudal anterior cingulate cortex thickness. $\uparrow$ age $\sim \downarrow$ left MFG thickness.

OCD: $\uparrow$ corpus callosum (except the isthmus). $\uparrow$ CC area, genu, anterior body, posterior body, isthmus and anterior splenium $\sim \uparrow$ compulsive symptom severity

OCD: No significant change in thalamic volume after CBT

MDD: $\downarrow$ left and right amygdala volumes. No correlations with symptom severity, age of onset or illness duration.

GAD: $\uparrow$ amygdala and DMPFC volumes. $\uparrow$ symptom severity $\uparrow$ DMPFC and ACC volumes

BPD: Ziprasidone treatment $\sim \uparrow$ in right BA 11 and 47 activation. No association with symptom improvement. $\downarrow$ BL right BA 47 activation $\sim \uparrow$ improvement of YMRS score.

BPD-r: $\downarrow$ BL GIX in LVLPFC. Change in LVLPFC Glu change in YMRS score

Imaging: fMRI BPD-RE: $\uparrow$ connectivity of Clinical:KSADS, the amygdala before and YMRS, CDRS R after treatment compared to YMRS, CDRS-R $\quad$ BPD-NRE. $\uparrow$ right amygdala functional connectivity after treatment $\sim \uparrow$ improvement in mania symptoms

Imaging: SMRI MDD: $>3$ untreated depressive episodes $\sim \downarrow$

GAF, HDRS, SCID subcallosal gyrus volumes compared to HC.

OCD: $\uparrow$ symptom severity $\sim \uparrow \mathrm{GM}$ volume in right Clinical: YBOCS insula, posterior orbitofrontal cortex, brainstem and cerebellum,

Clinical: YBOCS, HDRS
OCD: $\uparrow$ clinical improvement $\sim \uparrow$ changes in bilateral dosal ACC and in the right middle occipital gyrus

\footnotetext{
Note. Sample: $A D H D$ attention deficit hyperactivity disorder, $A N X$ anxiety disorder, $A U D$ alcohol use disorder, BPD bipolar disorder, BPD-I bipolar disorder I, BPD-O bipolar disorder with obesity, BPD-L bipolar disorder with low alcohol use, BPD-H bipolar disorder with high alcohol use, BPD-N bipolar disorder without obesity, $B P D-N O S$ bipolar disorder not otherwise specified, $B P D-R$ bipolar disorder remitters, BPD-NR bipolar disorder non remitters, BPD-RE bipolar disorder responders to pharmacotherapy, BPD-NRE bipolar disorder non responders to pharmacotherapy, $D D$ depressive disorder, $C U$ cannabis user, GAD generalised anxiety disorder, $g S P$ generalised social phobia, HC healthy controls, HC-O healthy controls with obesity, HC-N healthy controls without obesity, MDD major depression disorder, MDDx
} 
major depression disorder with borderline personality disorder, MHP mental health patients (mixed diagnosis sample), MT childhood maltreatment, OCD obsessive compulsive disorder, $P C$ psychiatric control (i.e. psychiatric diagnosis but no suicide attempt), PSD psychotic spectrum disorder, SA suicide attempters, ST stage of illness; 1B, 2, \& 3, SAD social anxiety disorder

Measures: $A A S$ anxiety analogue scale, $A C Q$ alcohol consumption questionnaire, $A D I S$ anxiety disorders interview schedule, $A S I$ addictions severity index, $A U D I T$ alcohol use disorder identification test, $B D I$ beck depression inventory, $B M I$ body mass index, $P R S$ brief psychiatric rating scale, $C A L S$ child affect liability scale, $C B C L$ child behaviour checklist, CDI children's depression inventory, CDRS children's depression rating scale; $R$ revised, CGAS children's global assessment scale, CGI clinical global impression scale, ChIPS children's interview for psychiatric syndromes, CDDR customary drinking and drug use record, CGAS child global assessment scale, C-CASA Columbia Classification Algorithm of Suicide Assessment, CSHF Colombia Suicide History Form, DTI diffuse tensor imaging, DUSI drug use screening inventory, $F H-R D C$ family history-research diagnostic criteria, $F M R I$ functional magnetic resonance imaging, GAF global assessment of functioning, $H A M A$ Hamilton anxiety rating scale, HDRS Hamilton depression rating scale, K-SADS kiddie schedule for affective disorders and schizophrenia, $L H A U D$ lifetime history of alcohol use disorder, LIFE modified longitudinal interval follow-up examination, LOI-CV Leyton Obsessive Inventory-Child Version, LSAS Liebowitz social anxiety scale, MASC multidimensional anxiety scale for children, MCQ meta cognition questionnaire, MFQ Mood frequencies questionnaire, MRS magnetic resonance spectroscopy, $P E T$ positron emission tomography, PRS Pfeffer rating scale, OCDAS obsessive compulsive disorder analogue scale, RCADS the revised child anxiety and depression scale, SAC substance abuse course-modified life II, SCARED screen for child anxiety related disorders, SCID structured clinical interview for DSM, SIQ suicide ideation questionnaire, SIS suicide intent scale, SOFAS social and occupational functioning assessment scale, sMRI structural magnetic resonance imaging, STAI-C state- trait anxiety inventory - child version, TLFB time-line followback, VBM voxel-based morphometry, Y-BOCS Yale-Brown obsessive-compulsive scale, YMRS young mania rating scale, YSRS the youth self-report scale

Findings: $\uparrow=$ Increased, Improved or Higher, $\downarrow=$ Decreased, Reduced or Lower, $\sim=$ 'is associated with', ACC anterior cingulate cortex, AD Axial diffusivity, ACG Anterior Cingulate Gyrus, BA Broadman Area -24, BL baseline, CV cerebellar vermis, DmPFC dorsomedial prefrontal cortex, DWMH deep white matter hyperintensities, FA fractional anisotropy, GABA gamma-aminobutyric acid, GM grey matter, GMV grey matter volumes, GSH glutathione, iFC intrinsic functional connectivity, LFG left fusiform gyrus, MD mean diffusivity, MFG middle frontal gyus, MPFC medial prefrontal cortex, OFC orbitofrontal cortex, RD radial diffusivity, $P V H$ periventricular hyperintensities, PCG precentral Gyrus, PFC prefrontal cortex, TBV total brain volumes, TLV temporal lobe volume, VMPFC ventromedial prefrontal cortex, WMH white matter hyperintensities, WMV White Matter Volumes

*indicates that the study features more than once in the data synthesis

with executive function deficits in these patients [53]. It is clear that the literature for anxiety disorders is less consistent with regard to the pattern of cognitive deficits and their relationship to anxiety and the severity of illness. The findings regarding GAD and cognition are unsurprisingly similar to the cognitive deficits observed in depression, and provide support for a shared underlying neurobiology for these disorders [54].

Clinical trials utilizing neuropsychological function as an outcome measure have demonstrated that verbal memory improves following: (i) lamotrigine treatment in bipolar patients [55]; and (ii) Transcranial Magnetic Stimulation (TMS) treatment in depressed patients [56]. Furthermore, the improved verbal memory performance was significantly associated with improvements in clinical symptoms of mania and depression, in the former study, and with reductions in hallmark symptoms of depression, in the latter. This adds to the close and complex relationship observed between cognition and mood disorders in young people, and reiterates the need for future research to closely examine the direction of these relationships.

\section{Neuroimaging}

There were 62 studies (a total of 3069 participants; $55.5 \%$ female) that utilised neuroimaging and across these studies $62 \%(1894 / 3069)$ were patients and $38 \%$ $(1175 / 3069)$ were healthy controls. Among the patient group $28 \%(534 / 1894)$ had depression, $27 \%$ (520/1894) had bipolar, $15 \%(288 / 1894)$ had anxiety, and $29 \%$ $(552 / 1894)$ were classified as other (i.e. mixed psychiatric samples, ADHD, alcohol use disorders, substance use disorder).

\section{Functional domains: social and economic participation, physical health, suicide and self-harm \& alcohol and substance use}

Results indicate that neuroimaging is a particularly useful modality for investigating suicide and self-harm behaviours as well as alcohol and substance use. In contrast, the utility of neuroimaging for investigating social and economic participation [57] and physical health [58] outcomes is yet to be determined due to a lack of studies exploring these relationships. Moreover, consistent with the findings previously discussed (see 'Neuropsychology') linking poor cognitive flexibility and decision making to suicidal behaviours, reduced Anterior Cingulate Cortex (ACC) volume was associated with a higher number of suicide attempts in patients with comorbid MDD and borderline personality disorder [59]. Furthermore, a study investigating ACC function using fMRI demonstrated that individuals with a suicide attempt history had increased dorsal ACC activity when viewing angry faces, and reduced visual, sensory, prefrontal ACC activity to intense happy and neutral faces compared to both healthy and psychiatric controls [60]. It is suggested that the ACC is an important area involved in attentional control that regulates both cognitive and emotional processes [61]. Structural and functional abnormalities in this area may be indicative of attentional control deficits that affect normal cognitive and emotional processes that are associated with an increased risk for suicidal behaviours in this population. However further evidence for this theory is needed since some fMRI studies could not distinguish between suicide attempters and healthy controls using similar decision making and cognitive flexibility tasks. Psychiatric controls demonstrated increased activity in the ACC during the go-no go task [62] and hippocampus during the 
Table 5 Sleep-wake and circadian biology studies evaluating the five functional domains in young people (12-30 yrs) with a mood and/or anxiety disorder

\begin{tabular}{|c|c|c|c|c|c|c|}
\hline $\begin{array}{l}\text { Outcome } \\
\text { measure }\end{array}$ & Study & Age (mean $\pm S D$ ) & Sample (N) & Aims & Key measures & Key findings \\
\hline \multirow[t]{4}{*}{$\begin{array}{l}\text { Social and } \\
\text { economic } \\
\text { participation }\end{array}$} & \multirow[t]{2}{*}{ [103] } & \multirow[t]{2}{*}{ PD: $30.6 \pm 6.1$} & \multirow[t]{2}{*}{$\mathrm{PD}(8 \mathrm{M} ; 12 \mathrm{~F})$} & \multirow[t]{2}{*}{$\begin{array}{l}\text { Determine whether HPA } \\
\text { activity can predict FUP } \\
\text { functional status. }\end{array}$} & $\begin{array}{l}\text { SWC: 24-hour } \\
\text { cortisol sam- } \\
\text { ples, ACTH } \\
\text { profiles, CRH } \\
\text { stimulation } \\
\text { test }\end{array}$ & \multirow[t]{2}{*}{$\begin{array}{l}\text { PD: } \uparrow \text { cortisol secretion pre- } \\
\text { treatment } \sim \downarrow \text { social and eco- } \\
\text { nomic participation (better } \\
\text { than pre-treatment clinical } \\
\text { severity) }\end{array}$} \\
\hline & & & & & $\begin{array}{l}\text { Functional: } \\
\text { SDS }\end{array}$ & \\
\hline & {$[102]^{*}$} & $\begin{array}{l}\operatorname{MDD}(M): 12.8 \pm 2.6 \\
\operatorname{MDD}(F): 13.6 \pm 1.9\end{array}$ & $\operatorname{MDD}(22 \mathrm{M} ; 33 \mathrm{~F})$ & $\begin{array}{l}\text { Investigate whether diurnal } \\
\text { changes in cortisol and DHEA } \\
\text { levels are associated with the } \\
\text { occurrence of undesirable life } \\
\text { events. }\end{array}$ & $\begin{array}{l}\text { SWC: } \\
\text { Cortisol/ } \\
\text { DHEA ratio, } \\
\text { Functional: } \\
\text { Semi- } \\
\text { structured } \\
\text { interview }\end{array}$ & $\begin{array}{l}\text { MDD: } \uparrow \text { cortisol/DHEA ratios } \\
\text { at } B L \sim \downarrow \text { social and } \\
\text { economic participation at } \\
\text { FUP. }\end{array}$ \\
\hline & [104] & $\begin{array}{l}\text { MHP: } 12.1 \\
\text { (7 - } 17.9 \text { years })\end{array}$ & MHP (62M; 40F) & $\begin{array}{l}\text { Investigate whether cortisol } \\
\text { reactivity is associated with } \\
\text { internalizing problem } \\
\text { behaviour }\end{array}$ & $\begin{array}{l}\text { SWC: Cortisol } \\
\text { level } \\
\text { Functional: } \\
\text { CBCL, SASC, } \\
\text { CDI }\end{array}$ & $\begin{array}{l}\text { MHP: } \uparrow \text { cortisol secretion } \\
\text { during the social interaction } \\
\text { task } \sim \downarrow \text { social and economic } \\
\text { participation }\end{array}$ \\
\hline \multirow{9}{*}{$\begin{array}{l}\text { Suicide and } \\
\text { self-harm }\end{array}$} & \multirow[t]{3}{*}[105]{$^{*}$} & \multirow{3}{*}{$\begin{array}{l}\text { MDD: } 25.19 \pm 2.42 \\
H C: 25.92 \pm 2.16\end{array}$} & MDD (33M; 23F) & \multirow{3}{*}{$\begin{array}{l}\text { Examine baseline } \\
\text { neuroendocrine predictors of } \\
\text { follow up clinical features }\end{array}$} & SWC: Sleep & \multirow{3}{*}{$\begin{array}{l}\text { MDD: } \uparrow \text { BL GH secretion } \\
\text { during first } 4 \text { hours of sleep } \\
\sim \text { a suicide attempt during } \\
\text { FUP }\end{array}$} \\
\hline & & & $\mathrm{HC}(10 \mathrm{M} ; 11 \mathrm{~F})$ & & $\begin{array}{l}\text { EEG, GH } \\
\text { secretion, } \\
\text { blood } \\
\text { cortisol }\end{array}$ & \\
\hline & & & & & $\begin{array}{l}\text { Functional: } \\
\text { Clinical } \\
\text { interview }\end{array}$ & \\
\hline & \multirow[t]{3}{*}[106]{} & \multirow{3}{*}{$\begin{array}{l}\text { MDD: } 25.19 \pm 2.42 \\
H C: 25.92 \pm 2.16\end{array}$} & MDD (33M; 23F) & \multirow{3}{*}{$\begin{array}{l}\text { Assess whether any } \\
\text { premorbid cortisol } \\
\text { abnormalities were associated } \\
\text { with depressive course of } \\
\text { illness }\end{array}$} & SWC: Sleep & \multirow{3}{*}{$\begin{array}{l}\text { MDD: } \uparrow \text { BL cortisol secretion } \\
\text { in the late evening hours } ~ \\
\text { suicide attempts during FUP }\end{array}$} \\
\hline & & & $\mathrm{HC}(10 \mathrm{M} ; 11 \mathrm{~F})$ & & $\begin{array}{l}\text { EEG, GH } \\
\text { secretion, } \\
\text { blood } \\
\text { cortisol }\end{array}$ & \\
\hline & & & & & $\begin{array}{l}\text { Functional: } \\
\text { Clinical } \\
\text { interview }\end{array}$ & \\
\hline & \multirow[t]{3}{*}[107]{$^{*}$} & MDD: $16 \pm 0.3$ & MDD (6M; 14F) & \multirow{3}{*}{$\begin{array}{l}\text { Compare sleep EEG profiles } \\
\text { of a sample of outpatient } \\
\text { adolescents }\end{array}$} & SWC: Sleep & \multirow{3}{*}{$\begin{array}{l}\text { MDD: } \downarrow \text { Delta sleep variable } \\
\sim \uparrow \text { suicidality (and } \\
\text { depression severity). }\end{array}$} \\
\hline & & \multirow[t]{2}{*}{$H C: 15.6 \pm 0.6$} & \multirow[t]{2}{*}{$\mathrm{HC}(7 \mathrm{M} ; 6 \mathrm{~F})$} & & $\begin{array}{l}\text { EEG, DIOOd } \\
\text { samples }\end{array}$ & \\
\hline & & & & & $\begin{array}{l}\text { Functional: } \\
\text { HDRS }\end{array}$ & \\
\hline \multirow[t]{6}{*}{$\begin{array}{l}\text { Clinical } \\
\text { syndrome }\end{array}$} & \multirow[t]{2}{*}[108]{} & \multirow[t]{2}{*}{ CS: $17.04 \pm 0.36$} & \multirow[t]{2}{*}{ CS (57M; 173F) } & \multirow{2}{*}{$\begin{array}{l}\text { Examine whether individual } \\
\text { differences in the CAR serve } \\
\text { as a premorbid risk factor for } \\
\text { MDD }\end{array}$} & $\begin{array}{l}\text { SWC: Salivary } \\
\text { cortisol }\end{array}$ & \multirow{2}{*}{$\begin{array}{l}\text { CS: } \uparrow \text { cortisol after waking at } \\
\mathrm{BL} \sim \uparrow \text { risk of developing } \\
\mathrm{MDD} \text { at FUP }\end{array}$} \\
\hline & & & & & $\begin{array}{l}\text { Clinical: SCID, } \\
\text { LSI }\end{array}$ & \\
\hline & [118] & HYP: $20.91 \pm 3.72$ & $\operatorname{HYP}(8 M ; 23 F)$ & $\begin{array}{l}\text { Assess circadian activity and } \\
\text { sleep in individuals at }\end{array}$ & $\begin{array}{l}\text { SWC: } \\
\text { Actigraphy }\end{array}$ & $\begin{array}{l}\text { HYP: } \uparrow \text { variability in duration, } \\
\text { fragmentation and efficiency }\end{array}$ \\
\hline & & $H C: 22.12 \pm 2.83$ & $\mathrm{HC}(8 \mathrm{M} ; 16 \mathrm{~F})$ & $\begin{array}{l}\text { behavioral high-risk of hypo- } \\
\text { mania/bipolar disorders }\end{array}$ & $\begin{array}{l}\text { Clinical: SCID, } \\
\text { HPS, HIQ, ISS }\end{array}$ & $\begin{array}{l}\text { of sleep, } \downarrow \text { sleep duration and } \\
\text { later more variable be times. }\end{array}$ \\
\hline & [120] & MDD: $12 \pm 1.9$ & $\operatorname{MDD}(2 \mathrm{M} ; 4 \mathrm{~F})$ & $\begin{array}{l}\text { Explore the effects of } \\
\text { fluoxetine on sleep EEG }\end{array}$ & $\begin{array}{l}\text { SWC: Sleep } \\
\text { EEG }\end{array}$ & $\begin{array}{l}\text { MDD: } \uparrow \text { stage } 1 \text { sleep, } \\
\text { arousals and REM density }\end{array}$ \\
\hline & & & & & $\begin{array}{l}\text { Clinical: K- } \\
\text { SADS, CDRS, } \\
\text { BDI, WSAS }\end{array}$ & fluoxetine treatment \\
\hline
\end{tabular}


Table 5 Sleep-wake and circadian biology studies evaluating the five functional domains in young people (12-30 yrs) with a mood and/or anxiety disorder (Continued)

\begin{tabular}{|c|c|c|c|c|c|}
\hline \multirow[t]{3}{*}[105]{$^{*}$} & MDD: $25.19 \pm 2.42$ & MDD (33M; 23F) & \multirow{3}{*}{$\begin{array}{l}\text { Examine baseline } \\
\text { neuroendocrine predictors of } \\
\text { follow up clinical features }\end{array}$} & \multirow{2}{*}{$\begin{array}{l}\text { SWC: Sleep } \\
\text { EEG, GH } \\
\text { secretion, } \\
\text { blood } \\
\text { cortisol }\end{array}$} & \multirow{3}{*}{$\begin{array}{l}\text { MDD: Premorbidly, earlier } \\
\text { and more steep GH secretion } \\
\text { at sleep onset }\end{array}$} \\
\hline & \multirow[t]{2}{*}{$H C: 25.92 \pm 2.16$} & \multirow[t]{2}{*}{$\mathrm{HC}(10 \mathrm{M} ; 11 \mathrm{~F})$} & & & \\
\hline & & & & $\begin{array}{l}\text { Clinical: } \\
\text { Clinical } \\
\text { interview }\end{array}$ & \\
\hline \multirow[t]{5}{*}{ [114] } & MDD: $17.04 \pm 0.35$ & MDD (4M; 7F) & \multirow{5}{*}{$\begin{array}{l}\text { Examine the associations } \\
\text { between MDD and anxiety } \\
\text { disorders, and HPA- axis } \\
\text { functioning }\end{array}$} & $\begin{array}{l}\text { SWC: Salivary } \\
\text { cortisol }\end{array}$ & \multirow[t]{5}{*}{$\begin{array}{l}\text { P-MDD \& MDD/ANX: flatter } \\
\text { diurnal cortisol slopes }\end{array}$} \\
\hline & ANX: $17.04 \pm 0.37$ & ANX (8M; 21F) & & \multirow{4}{*}{$\begin{array}{l}\text { Clinical: } \\
\text { MASQ, LSI }\end{array}$} & \\
\hline & $\begin{array}{l}\text { MDD/ANX: } 16.85 \pm \\
0.21\end{array}$ & $\begin{array}{l}\text { MDD-ANX } \\
(4 \mathrm{M} ; 8 \mathrm{~F})\end{array}$ & & & \\
\hline & P-MDD: $17.13 \pm 0.37$ & $\begin{array}{l}\text { P-MDD } \\
(11 \mathrm{M} ; 45 \mathrm{~F})\end{array}$ & & & \\
\hline & P-ANX: $17.02 \pm 0.38$ & P-ANX (6M; 2F) & & & \\
\hline \multirow[t]{2}{*}{ [109] } & HR: $16.8 \pm 1.7$ & $\mathrm{HR}(14 \mathrm{M} ; 15 \mathrm{~F})$ & \multirow{2}{*}{$\begin{array}{l}\text { Examine the cortisol increase } \\
\text { after awakening and basal } \\
\text { cortisol levels hypothesis that } \\
\text { high-risk offspring are more } \\
\text { reactive to psychosocial stress } \\
\text { than low-risk offspring }\end{array}$} & $\begin{array}{l}\text { SWC: Salivary } \\
\text { cortisol }\end{array}$ & \multirow[t]{2}{*}{$\begin{array}{l}\text { HR: } \uparrow \text { daytime cortisol in their } \\
\text { natural environment. }\end{array}$} \\
\hline & LR: $16.6 \pm 2.1$ & LR (14M; 15F) & & $\begin{array}{l}\text { Clinical: CDI, } \\
\text { CBCL, PANAS }\end{array}$ & \\
\hline \multirow[t]{2}{*}{ [110] } & HR: $18.3 \pm 2.6$ & $\mathrm{HR}(12 \mathrm{M} ; 12 \mathrm{~F})$ & \multirow{2}{*}{$\begin{array}{l}\text { Determine whether HR } \\
\text { individuals exhibit elevated } \\
\text { cortisol levels relative to } L R \\
\text { individuals during two weeks } \\
\text { of daily sampling }\end{array}$} & $\begin{array}{l}\text { SWC: Salivary } \\
\text { cortisol }\end{array}$ & \multirow[t]{2}{*}{$\begin{array}{l}\text { HR: } \uparrow \text { afternoon cortisol levels } \\
\text { in their natural environment }\end{array}$} \\
\hline & LR: $18.0 \pm 2.3$ & $\operatorname{LR}(11 \mathrm{M} ; 11 \mathrm{~F})$ & & $\begin{array}{l}\text { Clinical: BDI, } \\
\text { CDI, PSWQ, } \\
\text { CBCL, RLEQ }\end{array}$ & \\
\hline \multirow[t]{2}{*}[102]{$^{*}$} & MDD (M): $12.8 \pm 2.6$ & MDD (22M; 33F) & \multirow{2}{*}{$\begin{array}{l}\text { Investigate whether diurnal } \\
\text { changes in cortisol and DHEA } \\
\text { levels are associated with the } \\
\text { occurrence of undesirable life } \\
\text { events. }\end{array}$} & $\begin{array}{l}\text { SWC: } \\
\text { Cortisol/ } \\
\text { DHEA ratio, }\end{array}$ & \multirow[t]{2}{*}{$\begin{array}{l}\text { MDD: } \uparrow \text { cortisol/DHEA ratios } \\
\text { at BL } \sim \text { persistent major } \\
\text { depression at FUP }\end{array}$} \\
\hline & MDD (F): $13.6 \pm 1.9$ & & & $\begin{array}{l}\text { Clinical: } \\
\text { Semi- } \\
\text { structured } \\
\text { interview }\end{array}$ & \\
\hline \multirow[t]{3}{*}{ [113] } & $\begin{array}{l}\text { Mild: } 14.73 \pm 2.30 \\
\text { Moderate: } 15.69 \pm\end{array}$ & $\begin{array}{l}\text { Mild (10M; 20F) Moderate } \\
\text { (7M; 9F) }\end{array}$ & \multirow{3}{*}{$\begin{array}{l}\text { Examine cortisol reactivity to } \\
\text { a psychological stress } \\
\text { challenge in depressed } \\
\text { adolescents. }\end{array}$} & $\begin{array}{l}\text { SWC: Salivary } \\
\text { cortisol }\end{array}$ & \multirow{3}{*}{$\begin{array}{l}\text { Moderate/severe depression: } \\
\downarrow \text { cortisol response regardless } \\
\text { of child maltreatment history }\end{array}$} \\
\hline & & & & Clinical: & \\
\hline & Severe: $16.00 \pm 2.00$ & Severe (6M; 19F) & & $\begin{array}{l}\text { CECA, BDI-II, } \\
\text { K-SADS }\end{array}$ & \\
\hline \multirow[t]{2}{*}{ [119] } & \multirow[t]{2}{*}{ MDD: $23.94 \pm 2.31$} & \multirow[t]{2}{*}{ MDD (8M; 9F) } & \multirow{2}{*}{$\begin{array}{l}\text { Investigate the effect of } \\
\text { reducing slow waves during } \\
\text { sleep on depression } \\
\text { symptomology }\end{array}$} & $\begin{array}{l}\text { SWC: Sleep } \\
\text { EEG }\end{array}$ & \multirow{2}{*}{$\begin{array}{l}\text { MDD: } \uparrow \text { overnight dissipation } \\
\text { of SWA predicted } \downarrow \text { in } \\
\text { depressive symptoms. }\end{array}$} \\
\hline & & & & $\begin{array}{l}\text { Clinical: QIDS, } \\
\text { HDRS }\end{array}$ & \\
\hline \multirow[t]{3}{*}[107]{$^{*}$} & MDD: $16 \pm 0.3$ & MDD (6M; 14F) & \multirow{3}{*}{$\begin{array}{l}\text { Compare sleep EEG profiles } \\
\text { of a sample of outpatient } \\
\text { adolescents }\end{array}$} & SWC: Sleep & \multirow{3}{*}{$\begin{array}{l}\text { MDD: } \downarrow \text { Delta sleep variable } \\
\sim \uparrow \text { depression severity. }\end{array}$} \\
\hline & \multirow[t]{2}{*}{ HC: $15.6 \pm 0.6$} & \multirow[t]{2}{*}{$\mathrm{HC}(7 \mathrm{M} ; 6 \mathrm{~F})$} & & $\begin{array}{l}\text { EEG, blood } \\
\text { samples }\end{array}$ & \\
\hline & & & & $\begin{array}{l}\text { Clinical: } \\
\text { HDRS }\end{array}$ & \\
\hline \multirow[t]{2}{*}{ [117] } & DD: $15.35 \pm 1.85$ & $\mathrm{DD}(18 \mathrm{M} ; 28 \mathrm{~F})$ & $\begin{array}{l}\text { Assess sleep disturbances } \\
\text { pain and pubertal }\end{array}$ & $\begin{array}{l}\text { SWC: } \\
\text { Actigraphy }\end{array}$ & $\begin{array}{l}\text { DD: } \downarrow \text { sleep efficiency and } \\
\text { total time asleep, } \uparrow \text { time }\end{array}$ \\
\hline & HC: $14.83 \pm 1.76$ & $\mathrm{HC}(17 \mathrm{M} ; 43 \mathrm{~F})$ & $\begin{array}{l}\text { development in adolescent } \\
\text { depressive disorders }\end{array}$ & $\begin{array}{l}\text { Clinical: K- } \\
\text { SADS, PDS, } \\
\text { CES-D, BPD }\end{array}$ & $\begin{array}{l}\text { awake after sleep onset. } \uparrow \\
\text { pain intensity and depressive } \\
\text { symptoms predicted worse } \\
\text { sleep quality }\end{array}$ \\
\hline [111] & MDD: $22.4 \pm 1.5$ & MDD (9M; 17F) & $\begin{array}{l}\text { Examine the relationship } \\
\text { between longitudinal clinical }\end{array}$ & $\begin{array}{l}\text { SWC: Sleep } \\
\text { EEG }\end{array}$ & $\begin{array}{l}\text { MDD: recurrent illness } \sim \uparrow \\
\text { plasma cortisol near sleep }\end{array}$ \\
\hline & HC: $21.9 \pm 1.7$ & $\mathrm{HC}(13 \mathrm{M} ; 20 \mathrm{~F})$ & $\begin{array}{l}\text { course, sleep and cortisol in } \\
\text { adolescent depression }\end{array}$ & & \\
\hline
\end{tabular}


Table 5 Sleep-wake and circadian biology studies evaluating the five functional domains in young people (12-30 yrs) with a mood and/or anxiety disorder (Continued)

\begin{tabular}{|c|c|c|c|c|c|}
\hline & & & & $\begin{array}{l}\text { Clinical: K- } \\
\text { SADS }\end{array}$ & $\begin{array}{l}\text { HC: high density REM and } \downarrow \\
\text { REM latency at } B L \sim \text { the } \\
\text { development of depression a } \\
\text { FUP }\end{array}$ \\
\hline \multirow[t]{2}{*}{ [112] } & MDD: $15.6 \pm 1.4$ & MDD (6M; 10F) & \multirow{2}{*}{$\begin{array}{l}\text { Examine EEG sleep and HPA } \\
\text { changes during MDD } \\
\text { episodes and recovery }\end{array}$} & $\begin{array}{l}\text { SWC: NUFC, } \\
\text { sleep EEG }\end{array}$ & \multirow[t]{2}{*}{$\begin{array}{l}\text { MDD: } \downarrow \text { NUFC excretion } \\
\text { during remission }\end{array}$} \\
\hline & HC: $15.8 \pm 1.9$ & $\mathrm{HC}(7 \mathrm{M} ; 9 \mathrm{~F})$ & & $\begin{array}{l}\text { Clinical: PRS, } \\
\text { HDRS, K- } \\
\text { SADS }\end{array}$ & \\
\hline \multirow[t]{2}{*}{ [115] } & UPD: $21.8 \pm 4.3$ & UPD (5M; 13F) & \multirow{2}{*}{$\begin{array}{l}\text { Evaluate the potential of } \\
\text { circadian measures as early } \\
\text { markers of mood disorders } \\
\text { subtypes }\end{array}$} & $\begin{array}{l}\text { SWC: } \\
\text { Actigraphy, } \\
\text { DLMO }\end{array}$ & \multirow[t]{2}{*}{$\begin{array}{l}\text { BPD: } \downarrow \text { and later onset of } \\
\text { melatonin secretion }\end{array}$} \\
\hline & BPD: $22.8 \pm 4.8$ & $\mathrm{BPD}(3 \mathrm{M} ; 11 \mathrm{~F})$ & & $\begin{array}{l}\text { Clinical: } \\
\text { Psychiatric } \\
\text { interview } \\
\text { (DSM-IV } \\
\text { criteria), BDI }\end{array}$ & \\
\hline \multirow[t]{4}{*}{ [116] } & HC: $24.8 \pm 2.5$ & $\mathrm{HC}(8 \mathrm{M} ; 12 \mathrm{~F})$ & \multirow{4}{*}{$\begin{array}{l}\text { Investigate objectively the } 24- \\
\text { h sleep-wake cycle in adoles- } \\
\text { cents and young adults with } \\
\text { mood disorders }\end{array}$} & $\begin{array}{l}\text { SWC: } \\
\text { Actigraphy }\end{array}$ & \multirow{2}{*}{$\begin{array}{l}\text { BPD: } 62 \% \text { had delayed sleep } \\
\text { (during a depressive phase), } \\
\text { and later sleep offset } \\
\text { compared to UPD and HC }\end{array}$} \\
\hline & UPD: $20.1 \pm 4.7$ & \multirow[t]{3}{*}{ UPD and BPD (28M; 47F) } & & \multirow{3}{*}{$\begin{array}{l}\text { Clinical: } \\
\text { Psychiatric } \\
\text { interview } \\
\text { (DSM-IV } \\
\text { criteria) }\end{array}$} & \\
\hline & BPD: $23.2 \pm 4.3$ & & & & UPD: $30 \%$ had delayed sleep \\
\hline & & & & & HC: $10 \%$ had delayed sleep \\
\hline \multirow[t]{4}{*}{ [121] } & Stage 1a: $17.6 \pm 4.0$ & Stage 1a (7M; 11F) & \multirow{4}{*}{$\begin{array}{l}\text { Determine if disturbed sleep- } \\
\text { wake cycle patterns in young } \\
\text { people with emerging mental } \\
\text { disorder are associated with } \\
\text { stages of illness }\end{array}$} & $\begin{array}{l}\text { SWC: } \\
\text { Actigraphy }\end{array}$ & \multirow{2}{*}{$\begin{array}{l}\text { Stage } 1 \mathrm{~b} \& 2: \uparrow \text { delayed sleep } \\
\text { schedule, especially on } \\
\text { weeknights }\end{array}$} \\
\hline & Stage $1 \mathrm{~b}: 19.1 \pm 4.1$ & Stage 1b (44M; 38F) & & \multirow{3}{*}{$\begin{array}{l}\text { Clinical: } \\
\text { Psychiatric } \\
\text { interview } \\
\text { (DSM-IV } \\
\text { criteria) }\end{array}$} & \\
\hline & Stage $2+: 22.4 \pm 4.3$ & Stage 2+ (27M; 27F) & & & \multirow{2}{*}{$\begin{array}{l}\text { Stage } 1 \text { a \& } 2+\text { : } \downarrow \text { sleep } \\
\text { efficiency }\end{array}$} \\
\hline & HC: $24.4 \pm 3.1$ & $\mathrm{HC}(11 \mathrm{M} ; 12 \mathrm{~F})$ & & & \\
\hline
\end{tabular}

Note. Sample: ANX anxiety disorder, BPD bipolar disorder, CS community sample, DD depressive disorder, HC healthy controls, HR high risk participants (offspring of parents with bipolar disorder), HYP hypomanic participants, LR low risk participants (offspring of parents without a mental disorder), MDD-ANX comorbid Major depressive disorder and anxiety disorder, MDD major depression disorder, MHP mental health patients (mixed diagnosis sample), P-MDD past major depressive disorder, P-ANX past anxiety disorder, PD panic disorder

Measures: ACTH adrenocorticotropic hormone, BDI beck depression inventory, BPD body pain diagram, CBCL child behaviour checklist, CDI children's depression inventory, CDRS children's depression rating scale, CECA childhood experience of case and abuse contextual semi-structured interview and rating system, CES-D Center for Epidemiologic Studies Depression, CRH corticotropin-releasing hormone, DHEA dehydroepiandrosterone, DLMO dim light melatonin onset, DSM-IV diagnostic and statistical manual of mental disorders IV, EEG electroencephalography, GH growth hormone, HDRS Hamilton depression rating scale, HPS hypomanic personality scale, HIQ hypomanic interpretations questionnaire, ISS internal state scale, K-SADS schedule for affective disorders and schizophrenia for school age children, LSI life stress interview, MASQ mood and anxiety symptom questionnaire, NUFC nocturnal urinary free cortisol, PANAS positive and negative affect scale, PDS pubertal developmental scale, PRS Pfeffer rating scale, PSWQ Penn state worry questionnaire, QIDS quick inventory of depressive symptomatology, RLEQ recent life events questionnaire, SASC social anxiety scale for children, SCID structured clinical interview for DSM, SDS Sheehan disability scale, SWC sleepwake and circadian biology, WSAS work and social adjustment scale

Findings: $\uparrow=$ Increased, Improved or Higher, $\downarrow=$ Decreased, Reduced or Lower, $\sim=$ 'is associated with', BL baseline, FUP follow-up, NUFC nocturnal urinary free cortisol, REM rapid eye movement, SWA slow wave activity

*indicates that the study features more than once in the data synthesis

Iowa Gambling Task (IGT) [34], compared to suicide attempters who were comparable to healthy controls. It may be that the ACC is associated with attentional control related to the emotive processing that has been linked to suicide rather than the higher cognitive processes investigated in these latter studies.

Alcohol and substance use appears to affect multiple brain structures with most studies indicating that alcohol and substance use is associated with a pattern of reductions in brain volume and impairments in brain function. Cannabis use was investigated by two structural MRI (sMRI) studies where it was associated with reduced total white matter volumes [63], and reduced left fusiform gyrus grey mater volumes [64]. Whilst, the only fMRI study investigating cannabis use reported that lower amygdala reactivity was associated with higher rates of cannabis use in MDD patients [65]. One study [66] investigated alcohol use via SMRI and identified that lower prefrontal cortex white matter and overall grey matter volumes were associated with greater levels of alcohol consumption. Collectively, these findings are mostly consistent with the aforementioned neuropsychological studies (see 'Neuropsychology') and previous evidence regarding the neurobiological effects of alcohol 
Table 6 Neurophysiological studies evaluating the five functional domains in young people (12-30 yrs) with a mood and/or anxiety disorder

\begin{tabular}{|c|c|c|c|c|c|c|}
\hline $\begin{array}{l}\text { Outcome } \\
\text { measure }\end{array}$ & Study & Age $($ mean $\pm S D)$ & Sample (N) & Aims & Key measures & Key findings \\
\hline \multirow{2}{*}{$\begin{array}{l}\text { Social and } \\
\text { economic } \\
\text { participation }\end{array}$} & \multirow[t]{2}{*}{ [190] } & \multirow[t]{2}{*}{ MHP: $22.1 \pm 4.0$} & BPD (18) & \multirow{2}{*}{$\begin{array}{l}\text { Determine the longitudinal } \\
\text { relationship between } \\
\text { MMN/P3a and functional } \\
\text { outcomes in patients. }\end{array}$} & Na: MMN & \multirow{2}{*}{$\begin{array}{l}\text { BPD \& PSD: } \uparrow \text { BL MMN } \sim \uparrow \text { social and } \\
\text { economic participation at FUP }\end{array}$} \\
\hline & & & PSD (13) & & $\begin{array}{l}\text { Functional: } \\
\text { SOFAS, WHO- } \\
\text { DAS-II }\end{array}$ & \\
\hline \multirow{2}{*}{$\begin{array}{l}\text { Physical } \\
\text { health }\end{array}$} & \multirow[t]{2}{*}[191]{$^{*}$} & \multirow[t]{2}{*}{ MDD: $17.1 \pm 0.6$} & \multirow[t]{2}{*}{ MDD (8F) } & \multirow{2}{*}{$\begin{array}{l}\text { Investigate the effect of } \\
\text { nicotine on resting EEG } \\
\text { activity and affect. }\end{array}$} & $\mathrm{Na}: \mathrm{EEG}$ & \multirow{2}{*}{$\begin{array}{l}\text { MDD: } \downarrow \text { rPR theta \& } \downarrow \text { smoking } \\
\text { withdrawal, craving and physical } \\
\text { symptoms } \sim \text { acute nicotine } \\
\text { administration. }\end{array}$} \\
\hline & & & & & Functional: HONC & \\
\hline \multirow[t]{7}{*}{$\begin{array}{l}\text { Suicide and } \\
\text { self-harm }\end{array}$} & \multirow[t]{2}{*}{ [122] } & \multirow[t]{2}{*}{$\begin{array}{l}\text { SA: } 29.5 \pm 13.3, \mathrm{HC}: \\
34 \pm 13.3\end{array}$} & SA (24M; 16F) & \multirow{2}{*}{$\begin{array}{l}\text { Investigate the trait } \\
\text { predisposing to DSH by } \\
\text { examining EEG and } \\
\text { peripheral monoamine } \\
\text { activity. }\end{array}$} & $\begin{array}{l}\text { Na: EEG and } \\
\text { blood samples }\end{array}$ & \multirow[t]{2}{*}{$\begin{array}{l}\text { SA: } \downarrow \text { CNV and whole blood 5-HT } \\
\text { multiple episodes of self-harm. }\end{array}$} \\
\hline & & & $\mathrm{HC}(13 \mathrm{M} ; 14 \mathrm{~F})$ & & $\begin{array}{l}\text { Functional: HLS, } \\
\text { MADRS, SIS }\end{array}$ & \\
\hline & \multirow[t]{2}{*}{ [123] } & SA: 14 (12 - 17yrs) & SA (16F) & \multirow{2}{*}{$\begin{array}{l}\text { Examine EEG alpha } \\
\text { asymmetry among high- } \\
\text { risk adolescents }\end{array}$} & $\begin{array}{l}\mathrm{Na}: \mathrm{EEG} \text { alpha } \\
\text { asymmetry }\end{array}$ & \multirow{2}{*}{$\begin{array}{l}\text { SA: } \uparrow \text { posterior alpha asymmetry } \\
\text { suicidal intent (not depression } \\
\text { severity) }\end{array}$} \\
\hline & & HC: 14 (12 - 17yrs) & $\mathrm{HC}(22 \mathrm{~F})$ & & $\begin{array}{l}\text { Functional: HASS, } \\
\text { SIS }\end{array}$ & \\
\hline & \multirow[t]{3}{*}{ [124] } & $\begin{array}{l}\text { rMDD + CSA: } \\
31.60 \pm 10.98\end{array}$ & $\mathrm{rMDD}+\mathrm{CSA}(15 \mathrm{~F})$ & \multirow{3}{*}{$\begin{array}{l}\text { Examine the association } \\
\text { between CSA, MDD and } \\
\text { maladaptive behaviour. }\end{array}$} & $\mathrm{Na}: \mathrm{EEG}$ & \multirow{3}{*}{$\begin{array}{l}\text { rMDD + CSA: } \uparrow \text { subgenual ACC } \\
\text { activation during reward based } \\
\text { decision making, } \downarrow \text { reaction time } \\
\text { during incentive-based trials } \sim \uparrow \text { fre- } \\
\text { quency of self harm/suicidal } \\
\text { behaviours. }\end{array}$} \\
\hline & & rMDD: $24.81 \pm 3.94$ & rMDD: (16F) & & Functional: YRBS & \\
\hline & & HC: $30.44 \pm 10.78$ & $\mathrm{HC}(18 \mathrm{~F})$ & & (adult version) & \\
\hline \multirow{8}{*}{$\begin{array}{l}\text { Alcohol and } \\
\text { substance use }\end{array}$} & \multirow[t]{4}{*}{ [192] } & BPD-L: $21.8 \pm 3.9$ & BPD-L (5M; 11F) & \multirow{4}{*}{$\begin{array}{l}\text { Investigate the effects of } \\
\text { alcohol use on MMN in BP. }\end{array}$} & Na: MMN & \multirow[t]{4}{*}{ BPD-H: $\downarrow$ temporal MMN } \\
\hline & & BPD-H: $22.6 \pm 3.4$ & BPD-H (9M; 17F) & & Functional: AUDIT & \\
\hline & & HC-L: $22.4 \pm 2.6$ & HC-L (6M; 14F) & & & \\
\hline & & $\mathrm{HC}-\mathrm{H}: 23.4 \pm 3.2$ & $\mathrm{HC}-\mathrm{H}(6 \mathrm{M} ; 8 \mathrm{~F})$ & & & \\
\hline & \multirow[t]{4}{*}{ [193] } & AD: $24 \pm 3.77$ & $\mathrm{AD}(44 \mathrm{M} ; 47 \mathrm{~F})$ & \multirow{4}{*}{$\begin{array}{l}\text { Explore the use of a startle } \\
\text { paradigm and its } \\
\text { association with alcohol } \\
\text { use. }\end{array}$} & Na: Startle, ERP & \multirow{4}{*}{$\begin{array}{l}\text { AD: } \uparrow \text { facilitation, } \downarrow \text { inhibition of the } \\
\text { N4S component by pre pulse } \\
\text { stimuli. }\end{array}$} \\
\hline & & BD: $24.6 \pm 5.76$ & $\mathrm{BD}(23 \mathrm{M} ; 18 \mathrm{~F})$ & & Functional: & \\
\hline & & AFF: $22.9 \pm 3.94$ & AFF (32M; 65F) & & & \\
\hline & & DD: $23.5 \pm 3.17$ & $\mathrm{DD}(51 \mathrm{M} ; 61 \mathrm{~F})$ & & & \\
\hline $\begin{array}{l}\text { Clinical } \\
\text { syndrome }\end{array}$ & [128] & ANX : $12.9 \pm 2.6$ & ANX (7M; 13F) & $\begin{array}{l}\text { Examine the relationship } \\
\text { between ASR, symptom }\end{array}$ & $\begin{array}{l}\text { Na: Multiple } \\
\text { muscle ASR }\end{array}$ & $\begin{array}{l}\text { ANX: } \downarrow \text { in multiple muscle ASR } \sim \downarrow \text { in } \\
\text { anxiety symptoms. }\end{array}$ \\
\hline & & HC: $12.0 \pm 2.5$ & $\mathrm{HC}(10 \mathrm{M} ; 15 \mathrm{~F})$ & $\begin{array}{l}\text { reduction and treatment } \\
\text { success. }\end{array}$ & $\begin{array}{l}\text { Clinical: ADIS-C/P, } \\
\text { SCAS }\end{array}$ & $\begin{array}{l}\text { ANX: } \uparrow \text { multiple muscle ASR } \\
\text { predicted CBT treatment response }\end{array}$ \\
\hline & [133] & OCD: $13.9 \pm 2.4$ & OCD (18M; 22F) & Assess ERN as a biomarker & Na: ERN & OCD \& SIB: $\uparrow$ ERN at Cz \\
\hline & & SIB: $13.9 \pm 2.4$ & $\operatorname{SIB}(13 \mathrm{M} ; 6 \mathrm{~F})$ & & Clinical: Y-BOCS, & $\begin{array}{l}\text { Independent of symptom severity, } \\
\text { current diagnostic status and }\end{array}$ \\
\hline & & HC: $13.8 \pm 2.3$ & $\mathrm{HC}(20 \mathrm{M} ; 20 \mathrm{~F})$ & & & treatment effects). \\
\hline & [134] & ANX: $11.8 \pm 2.3$ & ANX (3M; 10F) & Demonstrate ERN & Na: ERN & ANX \& OCD: $\uparrow$ ERN at Cz \\
\hline & & OCD: $12.7 \pm 2.2$ & OCD (8M; 18F) & $\begin{array}{l}\text { amplitude is increased in } \\
\text { young anxiety patients. }\end{array}$ & Clinical: Y-BOCS, & $\begin{array}{l}\text { (Independent of symptom severity, } \\
\text { current diagnostic status and }\end{array}$ \\
\hline & & HC: $12.4 \pm 2.2$ & $\mathrm{HC}(14 \mathrm{M} ; 13 \mathrm{~F})$ & & & treatment effects). \\
\hline & [194] & RES: $14.1 \pm 2.8$ & RES (2M; 6F) & Examine the relationship & Na: TMS & NoRES: $\uparrow$ deficits in pre-treatment \\
\hline & & NoRES: $13.1 \pm 1.6$ & NoRES (5M; 3F) & $\begin{array}{l}\text { between IMS with } \\
\text { subsequent treatment } \\
\text { response }\end{array}$ & $\begin{array}{l}\text { Clinical: CDRS-R, } \\
\text { QIDS, CGI-severity } \\
\text { scale }\end{array}$ & \\
\hline & [195] & HC: $25.54 \pm 3.41$ & $\mathrm{HC}(28 \mathrm{M} ; 16 \mathrm{~F})$ & $\begin{array}{l}\text { Investigate the intensity } \\
\text { evaluation of social stimuli }\end{array}$ & $\begin{array}{l}\text { Na: } \\
\text { ERP (N170, P1, P2) }\end{array}$ & $\begin{array}{l}\text { MDD: } \uparrow \text { intensity scores for sad faces } \\
\text { compared with } \mathrm{HC}, \uparrow \text { reaction times }\end{array}$ \\
\hline & & DEP: $25.96 \pm 4.58$ & DEP (9M; 15F) & in depression & $\begin{array}{l}\text { Clinical: SCID, BDI, } \\
\text { RDRS RAI }\end{array}$ & amplitude for sad faces \\
\hline & & MDD: $26.58 \pm 4.16$ & $\operatorname{MDD}(10 \mathrm{M} ; 14 \mathrm{~F})$ & & & $\begin{array}{l}\text { DEP: } \downarrow \text { scores for happy and neutral } \\
\text { faces, } \uparrow \text { reactions times and } \uparrow P 1 \&\end{array}$ \\
\hline
\end{tabular}


Table 6 Neurophysiological studies evaluating the five functional domains in young people (12-30 yrs) with a mood and/or anxiety disorder (Continued)

$$
\begin{aligned}
& \text { [196] HC: } 27.7 \pm 7.0 \\
& \mathrm{HC}(14 \mathrm{M} ; 12 \mathrm{~F}) \\
& \text { BPD: } 30.7 \pm 6.1 \\
& \text { BPD (10M; 19F) } \\
& \text { [135] OCD: } 13.3 \pm 2.8 \text {, } \\
& \text { OCD (13M; 5F) } \\
& \text { HC: } 11.9 \pm 2.6 \\
& \text { HC (8M; 10F) } \\
& \text { [197] HC: } 17 \pm 1.6 \\
& \text { HC (43F) } \\
& \text { HC-FHA: } 16.5 \pm 1.3 \text { HC-FHA (31F) } \\
& \text { HC-FHD: } 16.1 \pm 1.5 \quad \text { HC-FHD (27F) } \\
& \text { DEP: } 17.2 \pm 1.4 \quad \text { DD (12F) } \\
& \text { DEP-FHA: } 17.3 \pm 1.5 \text { DD-FHA (9F) } \\
& \text { DEP-FHD: } 16.3 \pm 1.3 \text { DD-FHD (8F) } \\
& \text { [191]* MDD: } 17.1 \pm 0.6 \quad \text { MDD (8F) }
\end{aligned}
$$

\section{[129] MDD: $30.4 \pm 11.8$}

$\operatorname{MDD}(28 \mathrm{M} ; 23 \mathrm{~F})$

$\begin{array}{lll}{[167]^{*}} & \text { OCD } 27 \pm 9.8 & \text { OCD (15M; 16F) } \\ & \text { HC: } 27.4 \pm 9.1 & \text { HC (14M; 16F) } \\ & & \\ {[52]^{*}} & \text { OCD: } 24.06 \pm 5 & \text { OCD (21M; 9F) } \\ & \text { HC: } \text { Matched } & \text { HC (21M; 9F })\end{array}$

[136] OCD-U: $25 \pm 8.0$

OCD-U (9M; 10F)

OCD-M: $30.8 \pm 9.5$

OCD-M (9M; 10F)

PC-M: $31.7 \pm 10.6$

PC-M (8M; 11F)

HC: $25.3 \pm 7.5$

$\mathrm{HC}(11 \mathrm{M} ; 10 \mathrm{~F})$

[127] DEP: $20.9 \pm 0.55$
Assess brain function impairments in bipolar patients.

Examine ERN in paediatric patients with OCD

Evaluate the effects of depression and a family history of alcohol or substance dependence on P300.

Investigate the effect of acute nicotine administration on resting EEG activity and affect

Assess the utility of baseline LDAEP predicting response to antidepressants.

Characterize the cognitive functions of the patients with OCD by utilizing ERPs and neuropsychological tests

Assess the relationship between cognitive dysfunction, clinical status and severity in OCD.

Examine the effects of chronic medication on error responses in OCD.

Examine whether recurrent
major depression is associated
with abnormal startle

P2 amplitude for happy faces
compared to sad faces.

Na: Resting EEG

BPD: $\uparrow$ power in all wave bands. Clinical: BDI_ Marked increases in right temporal Na: ERN

Clinical : Y-BOCS theta and left occipital beta.

OCD: $\uparrow$ ERN pre-treatment and after treatment. No relationship with symptom severity or changes in symptom severity

Na: ERP (P300) DD: $\downarrow$ P300 amplitude. No effect of Clinical: SSAGA, family history of alcohol or drug Clinical: SSAGA, dependence.
MAST, PANAS
Na: EEG

Clinical: BDI,

HONC, PANAS

Na: LDAEP

Clinical: HDRS, MADRS

Na: ERP (P300)

Clinical: HDRS

Na: ERP (N100, P200, N200, P300)

Clinical: YBOCS

Na: ERN

Clinical: HDRS, HAMA, YBOCS

Na: ASR

Clinical: SCID
MDD: Nicotine $\downarrow$ theta amplitude in right parietal region. No associations with mood.

MDD: steep N1 sLORETA-LDAEP at $\mathrm{BL} \sim$ treatment response. $\uparrow \mathrm{P} 2$ sLORETA-LDAEP slope at week 1 treatment response.

OCD: $\downarrow$ P300 duration. $\downarrow$ stroop duration $\uparrow$ P300 amplitude in occipital, parietal and temporal anterior regions.

OCD: $\uparrow$ P200 amplitude, unrelated to neither severity nor chronicity of illness. $\downarrow$ N200 amplitude (worsens with $\uparrow$ severity). $\downarrow$ N100 and P200 $\sim \uparrow$ chronicity

OCD: $\uparrow$ ERN, irrespective of medication use.

HC \& PC: $\uparrow$ anxiety and depression $\sim \uparrow$ ERN amplitude

DEP: $\uparrow$ ASR was associated with multiple (more than 1) depressive episode.

\footnotetext{
Note. Sample : AFF affective disorder (not specified), AD alcohol dependence, ANX anxiety disorder, BD behavioural disorder, BPD bipolar disorder, BPD-L bipolar disorder with low alcohol use, BPD-H bipolar disorder with high alcohol use, DD depressive disorder, DD-FHA depressive disorder with family history of alcohol dependence, DD-FHD depressive disorder with family history of drug dependence, DrDep drug dependence, HC healthy controls, HC-FHA healthy control with family history of alcohol dependence, HC-FHD healthy control with family history of drug dependence, HC-L health control with low alcohol use, HC-H healthy control with high alcohol use, MDD major depression disorder, MHP mental health patients (mixed diagnosis sample), NoRES treatment non responders, OCD obsessive compulsive disorder, OCD-M obsessive compulsive disorder patient medicated, OCD-U obsessive compulsive disorder patients unmedicated, PC-M psychiatric control patient medicated, PSD psychotic spectrum disorder, RES treatment responders, rMDD remitted major depression disorder, rMDD+CSA remitted major depression disorder with childhood sexual abuse history, SA suicide attempters, SIB suicide ideation behaviour

Measures : ADIS-C/P anxiety disorders interview schedule for children, ASR auditory startle reflex, AUDIT Alcohol Use Disorder Identification Test, BAI beck anxiety inventory, BDI beck depression inventory, CBCL child behaviour checklist, CDI children's depression inventory, CDRS children's depression rating scale, CGI clinical global impression scale, EEG electroencephalography, ERP event related potential, ERN event related negativity, FHAM family history assessment module, HAMA Hamilton anxiety rating scale, HASS Harkavy Asnis suicide scale, HDRS Hamilton depression rating scale, HLS beck hopelessness scale, HONC hooked on nicotine checklist, LDAEP loudness dependant auditory evoked potential, MASC multidimensional anxiety scale for children, MADRS Montgomery-Asberg depression rating scale, MMN mismatch negativity, MAST Michigan Alco- holism Screening Test, PANAS positive and negative affect scale, QIDS quick inventory of depressive symptomatology, SCID structured clinical interview for DSM, SCAS Spence children's anxiety scale, SIS suicide intent scale, SOFAS social and occupational functioning assessment scale, SSAGA semi-structured assessment for the genetics of alcoholism, TMS transcranial magnetic stimulation, WHO-DAS-II World Health Organisation Disability Assessment Scale II, Y-BOCS, Yale-Brown obsessive-compulsive scale, YRBS youth risk behaviour survey

Findings : $\uparrow=$ Increased, Improved or Higher, $\downarrow=$ Decreased, Reduced or Lower, $\sim=$ 'is associated with', 5 -HT serotonin, BL baseline, CBT cognitive behaviour therapy, CNV contingent negative variation, FUP follow-up, N4S late wave frontal ERP component responses, rPR right Parietal Region, RT reaction time * indicates that the study features more than once in the data synthesis
} 
and substance misuse on the structure and function of frontal and temporal brain regions [67].

\section{Functional domain: clinical syndrome}

From the fifty neuroimaging studies investigating clinical syndrome, multiple regions of interest have been studied using a variety of imaging methods.

Structural magnetic resonance imaging (sMRI) In depressive disorders the majority of studies examining brain structure have focused on frontal and limbic regions with mixed findings; although some promising patterns emerge when the severity and/or clinical course of specific disorders are considered. Reduced ACC volumes [68, 69], and increased ACC thickness [70], have been identified in MDD patients compared to healthy controls. Whilst, no significant association with clinical severity or symptoms was found in these studies, reduced ACC volume was associated with higher borderline personality disorder symptom severity but not depression, in patients diagnosed with comorbid MDD and borderline personality disorder [59]. Some further lines of inquiry provide greater detail about how the severity of the clinical syndrome may influence or be influenced by particular brain structures. Decreased grey matter volume in frontal brain regions were evident in patients with a discrete or persistent affective illness compared to those with attenuated syndromes and healthy controls [71], and MDD patients who experienced more than three untreated depressive episodes had reduced subcallosal gyrus volumes, an ACC subdivision [72]. Collectively, these studies reiterate the relationship between reductions in the ACC and depression, and suggest that greater reductions in the ACC may be associated with more severe illness.

Compared to healthy controls, MDD patients had lower amygdala volumes, and no association with clinical severity or illness duration [73], however MDD patients near the onset of their illness had increased amygdala-hippocampal volume ratios that were associated with higher severity of anxiety, but not depression severity [74]. This is consistent with evidence indicating that larger amygdala volumes in GAD patients are associated with greater symptom severity [75]. This suggests that common forms of depression and anxiety may share similar biological processes and genetic liability [76, 77], yet differ in their phenotypic expression. Although distinct pathophysiology may underlie the development of SAD, since lower amygdala grey matter density was associated with greater disease duration and earlier age of onset in this group [78].

Changes to areas of the brain following a course of treatment can provide valuable insight into the success of treatment and how this may have influenced the course of illness. Compared to healthy controls, treatment naïve patients with OCD had larger thalamic volumes, which normalised following paroxetine treatment. These reductions were also associated with a decrease in OCD symptoms. While, CBT treatment for OCD was not associated with change in thalamic volumes [79], greater symptom improvement following CBT was associated with a normalised metabolism in the ACC [80], and with increased prefrontal grey matter volumes [81]. These studies are consistent with the association between the lower symptom severity and greater prefrontal grey matter volumes [82]. Higher compulsive symptom severity was associated with reduced pituitary gland volume in OCD patients in males, compared to healthy controls [83], and larger corpus callosum area [84]. Collectively these OCD studies seem to indicate that pharmacological treatment for OCD may be particularly useful for targeting deficits in thalamus structure and function, whilst CBT may be better for targeting clinical features associated with prefrontal structures.

Functional magnetic resonance imaging (fMRI) Studies investigating brain function using fMRI have also predominantly focused on frontal and limbic regions. Clinical improvement following lamotrigine treatment for bipolar disorder [85], and SSRI treatment for generalised social phobia [86], were associated with reductions in amygdala activation whilst viewing negative valanced emotional pictures. Similarly, higher activation of the amygdala to emotionally fearful faces compared to happy faces was associated with treatment response to CBT or medication in anxiety patients [87]. Bipolar disorder pharmacotherapy treatment responders compared to non-responders had greater amygdala functional connectivity within the frontolimbic network, and higher amygdala functional connectivity within this network after treatment was associated with greater improvements in mania symptoms [88]. These studies consistently demonstrate a relationship between higher amygdala activity and patterns of treatment response for both bipolar and anxiety disorders. This may be indicative of a shared neurobiological vulnerability for heightened amygdala reactivity associated with stress and the emergence of particular affective disorders [89].

Diffusion Tensor Imaging (DTI) The evidence from these DTI studies collectively indicate that poorer white matter integrity is associated with affective disorders that may be an early marker of disorder. A diagnosis of a depressive disorder, compared to healthy controls was associated with lower fractional anisotropy, a measure indicating poorer white matter integrity, and higher mean and radial diffusivity in the 
Table 7 Metabolic studies evaluating the five functional domains in young people (12-30 yrs) with a mood and/or anxiety disorder

\begin{tabular}{|c|c|c|c|c|c|c|}
\hline $\begin{array}{l}\text { Outcome } \\
\text { measure }\end{array}$ & Study & Age (mean $\pm S D)$ & Sample (N) & Aims & Key measures & Key findings \\
\hline \multirow{2}{*}{$\begin{array}{l}\text { Social and } \\
\text { participation }\end{array}$} & \multirow[t]{2}{*}[140]{$^{*}$} & \multirow[t]{2}{*}{ MHP: $28.74 \pm 10.38$} & MHP (38M; 28F) & \multirow{2}{*}{$\begin{array}{l}\text { Identify changes in the } \\
\text { rates of obesity in } \\
\text { never-treated patients } \\
\text { with mood disorder } \\
\text { over } 4 \text { years of follow- } \\
\text { up. }\end{array}$} & Metabolic: BMI & \multirow{2}{*}{$\begin{array}{l}\text { MHP: } \uparrow \mathrm{BMI} \sim \uparrow \text { social and } \\
\text { economic participation }\end{array}$} \\
\hline & & & (40 MDD, 26 BPD) & & Functional: GAF & \\
\hline \multirow{2}{*}{$\begin{array}{l}\text { Physical } \\
\text { health }\end{array}$} & \multirow[t]{2}{*}{ [198] } & $\mathrm{FH}+: 18.9 \pm 1.0$ & $\mathrm{FH}+(32 \mathrm{M}, 53 \mathrm{~F})$ & \multirow{2}{*}{$\begin{array}{l}\text { Determine whether } \\
\text { young people with a } \\
\text { family history of } \\
\text { depression have altered } \\
\text { metabolic markers. }\end{array}$} & \multirow{2}{*}{$\begin{array}{l}\text { Metabolic/Functional: } \\
\text { glucose, lipids and high- } \\
\text { sensitivity CRP. BP, arterial } \\
\text { stiffness and waking cor- } \\
\text { tisol concentration. }\end{array}$} & \multirow{2}{*}{$\begin{array}{l}\mathrm{FH}+: \uparrow \text { peripheral and central } \\
\mathrm{BP} \text {, arterial stiffness and } \downarrow \\
\text { insulin sensitivity }\end{array}$} \\
\hline & & HC: $19.1 \pm 0.1$ & $\mathrm{HC}(27 \mathrm{M} ; 42 \mathrm{~F})$ & & & \\
\hline \multirow[t]{12}{*}{$\begin{array}{l}\text { Suicide and } \\
\text { self-harm }\end{array}$} & \multirow[t]{4}{*}{ [138] } & SUC: $15.93 \pm 1.48$ & SUC (15M; 32F) & \multirow{2}{*}{$\begin{array}{l}\text { Examine the } \\
\text { relationship between } \\
\text { serum cholesterol levels } \\
\text { and suicidal behaviours }\end{array}$} & $\begin{array}{l}\text { Metabolic: blood serum } \\
\text { samples }\end{array}$ & \multirow{2}{*}{$\begin{array}{l}\text { SUC: } \uparrow \text { cholesterol } \sim \text { current } \\
\text { suicide behaviour (within the } \\
\text { SUC group, } \uparrow \text { serum } \\
\text { cholesterol } \sim \downarrow \text { severity of SUC, } \\
\text { but not } \sim \text { symptom severity) }\end{array}$} \\
\hline & & PC: $16.22 \pm 1.95$ & PC (58M; 47F) & & Functional: SPI & \\
\hline & & \multirow[t]{2}{*}{ PD: $25.3 \pm 3.3$} & \multirow[t]{2}{*}{ PD (37M; 35F) } & \multirow{2}{*}{$\begin{array}{l}\text { Elucidate the } \\
\text { relationships between } \\
\text { alexithymia, suicide } \\
\text { ideation and serum lipid } \\
\text { levels. }\end{array}$} & $\begin{array}{l}\text { Metabolic: BMI, blood } \\
\text { serum samples }\end{array}$ & \multirow[t]{2}{*}{$\begin{array}{l}\mathrm{PD}: \downarrow \mathrm{HDL}-\mathrm{C} \text { and } \uparrow \mathrm{VLDL}-\mathrm{C} \sim \\
\text { higher suicide ideation }\end{array}$} \\
\hline & & & & & Functional: SSI & \\
\hline & \multirow[t]{2}{*}{ [137] } & SA: $15.44 \pm 1.99$ & SA (17M; 49F) & \multirow{2}{*}{$\begin{array}{l}\text { Explore the associations } \\
\text { between cholesterol } \\
\text { and suicidal behaviour }\end{array}$} & $\begin{array}{l}\text { Metabolic: blood serum } \\
\text { samples }\end{array}$ & \multirow[t]{2}{*}{$\begin{array}{l}\text { SA: } \downarrow \text { cholesterol levels } \sim \\
\text { attempted suicide history }\end{array}$} \\
\hline & & PC: $15.19 \pm 1.68$ & PC (15M; 39F) & & $\begin{array}{l}\text { Functional: hospital } \\
\text { records }\end{array}$ & \\
\hline & \multirow[t]{2}{*}{ [199] } & SA: 16.8 SEM $=.74$ & SA (3M; 6F) & \multirow[t]{2}{*}{$\begin{array}{l}\text { Investigate platelet PBR } \\
\text { density in suicidal teens }\end{array}$} & $\begin{array}{l}\text { Metabolic: blood serum } \\
\text { samples }\end{array}$ & \multirow[t]{2}{*}{ SA: $\downarrow$ platelet PBR density } \\
\hline & & PC: 16.5 SEM $=.5$ & PC (7M; 3F) & & $\begin{array}{l}\text { Functional: hospital } \\
\text { records, SPI, SRS }\end{array}$ & \\
\hline & \multirow[t]{4}{*}{ [155] } & SA: $15.87 \pm 1.56$ & $\mathrm{SA}(10 \mathrm{M} ; 25 \mathrm{~F})$ & \multirow{4}{*}{$\begin{array}{l}\text { Evaluate the relationship } \\
\text { between plasma } \\
\text { serotonin levels and } \\
\text { psychometric measures } \\
\text { in suicidal adolescents }\end{array}$} & $\begin{array}{l}\text { Metabolic: blood serum } \\
\text { samples }\end{array}$ & \multirow{4}{*}{$\begin{array}{l}\text { SA: } \downarrow \text { plasma } 5-H T \text { level } \sim \uparrow \\
\text { suicidality. }(5-H T \text { did not dis- } \\
\text { criminate between the psychi- } \\
\text { atric diagnostic categories) }\end{array}$} \\
\hline & & PC: 16.291 .81 & PC (19M; 11F) & & Functional: hospital & \\
\hline & & ER: 16.912 .47 & ER (13M; 38F) & & & \\
\hline & & HC: 15.261 .41 & $\mathrm{HC}(45 \mathrm{M} ; 50 \mathrm{~F})$ & & & \\
\hline \multirow{2}{*}{$\begin{array}{l}\text { Alcohol and } \\
\text { substance } \\
\text { use }\end{array}$} & \multirow[t]{2}{*}[200]{} & BPD-O: $12.9 \pm 3.1$ & BPD-O (77M; 68F) & \multirow{2}{*}{$\begin{array}{l}\text { Investigate obesity in } \\
\text { paediatric bipolar } \\
\text { patients and notable } \\
\text { correlates }\end{array}$} & Metabolic: BMI & \multirow{2}{*}{$\begin{array}{l}\text { BPD: SUD } 2.8 \text { fold increased } \\
\text { prevalence of BPD-OB. }\end{array}$} \\
\hline & & BPD-NO: $13.3 \pm 3.0$ & BPD-NO (108M; 95F) & & Functional: K-SADS-P & \\
\hline \multirow{4}{*}{$\begin{array}{l}\text { Clinical } \\
\text { syndrome }\end{array}$} & \multirow[t]{2}{*}{ [162] } & MDD: $24.1 \pm 3.2$ & MDD (45M; 44F) & \multirow{2}{*}{$\begin{array}{l}\text { Examine the association } \\
\text { between } \mathrm{MDD} \text { in } \\
\text { childhood and BMI in } \\
\text { adulthood }\end{array}$} & Metabolic: BMI & MDD: $\uparrow$ BMI at FUP (in \\
\hline & & HC: $22.2 \pm 2.9$ & $\mathrm{HC}(43 \mathrm{M} ; 45 \mathrm{~F})$ & & Clinical: KSADS, & \\
\hline & {$[140]^{*}$} & MHP: $28.74 \pm 10.38$ & MHP (38M; 28F) & Identify changes in the & Metabolic: BMI & MHP: clinical improvement $\sim \uparrow$ \\
\hline & & & (40 MDD, 26 BPD) & $\begin{array}{l}\text { treated patients with } \\
\text { mood disorder over } 4 \\
\text { years of follow-up. }\end{array}$ & Clinical: HAMD & \\
\hline
\end{tabular}

Note. Sample : BPD bipolar disorder, BPD-O bipolar disorder with obesity, BPD-NO bipolar disorder without obesity, ER emergency room patients admitted for suicide attempt, $\mathrm{HC}$ healthy controls, FH+ family history of depression, MDD major depression disorder, MHP mental health patients (mixed diagnosis sample), PC psychiatric control (i.e. psychiatric diagnosis but no suicide attempt), PD panic disorder, SA suicide attempters, SUC suicidal tendencies (either ideation, threat or attempt)

Measures : BMI body mass index, BP blood pressure, CRP C-reactive protein, GAF global assessment of functioning, HDRS Hamilton depression rating scale, KSADS schedule for affective disorders and schizophrenia for school age children, SPI suicide potential interview, SRS suicide risk scale, SSI scale of suicide ideation Findings : $\uparrow=$ Increased, Improved or Higher, $\downarrow$ = Decreased, Reduced or Lower, $\sim$ = 'is associated with', 5-HT serotonin, FUP follow-up, PBR peripheral-type benzodiazepine receptors

* indicates that the study features more than once in the data synthesis 
corpus callosum, whilst higher fractional anisotropy and axial diffusivity and lower radial diffusivity in the uncinated fasciculus [90]. Lower fractional anisotropy in the ACC [91], and genu, body and splenium of the corpus callosum as well as the superior and anterior corona radiata [92] is evident in bipolar disorder compared to healthy controls. For those who experienced maltreatment during childhood, compared to healthy controls, MDD at follow up was associated with lower fractional anisotropy in the superior longitudinal fasciculi and the right cingulum-hippocampal projection, whilst substance use disorder at follow up was associated with lower fractional anisotropy in the right cingulum-hippocampal projection [93]. Greater obsession symptom severity in OCD patients was associated with higher fractional anisotropy in the splenium [94]. Lower white matter integrity in the genu of the corpus callosum, anterior thalamic radiation, anterior cingulum and sagittal stratum was associated with higher depression severity in MDD patients [95]. Having a discrete or persistent psychiatric illness was associated with lower fractional anisotropy in the left anterior corona radiata compared to healthy controls, whilst a similar pattern of lower fractional anisotropy within this region was associated with attenuated syndromes of psychiatric illness [96].

Magnetic Resonance Spectroscopy (MRS) In terms of MRS studies, the major metabolites that were investigated include $\mathrm{N}$-acetyl aspartate - a measure of neuronal integrity; choline - involved in cell membrane production, lactate - marks glycolysis has been initiated in an oxygen deficient environment; creatine - indicates metabolism of brain energy; glutamate/glutamine -an excitatory neurotransmitter involved in neural activation; and GABA - an inhibitory neurotransmitter involved in reducing neuronal excitability [97]. Lower total choline in the left striatum was associated with OCD and remained consistent over the course of illness [98]. Compared to healthy controls, bipolar disorder was associated with a higher lactate to $\mathrm{N}$ acetyl aspartate and lactate to creatine ratios [99], and higher N-acetyl aspartate in the ACC and higher $\mathrm{N}$-acetyl aspartate, choline and creatine in the ventral lateral prefrontal cortex [100]. Moreover, bipolar disorder responders had lower left ventral lateral prefrontal cortex glutamate/glutamine [101]. For both MDD patients and healthy controls, higher ACC GABA was associated with lower anhedonia scores, and lower ACC GABA was associated with MDD [68].

\section{Sleep-wake and circadian biology}

This section entails studies that have utilised either sleep physiology (e.g. sleep EEG) and/or sleep-wake monitoring (e.g. actigraphy) or indicators (e.g. cortisol secretion) to determine sleep-wake and circadian function. There were 23 studies (a total of 1609 participants; $59.3 \%$ female) that utilised sleep-wake and circadian biology and across these studies $84 \%$ $(1352 / 1609)$ were patients and $16 \%(257 / 1609)$ were healthy controls. Among the patient group $55 \%$ (747/1352) had depression, $3 \%(45 / 1352)$ had bipolar, $5 \%(69 / 1352)$ had anxiety, and $36 \%(491 / 1352)$ were classified as other.

\section{Functional domains: social and economic participation, physical health, suicide and self-harm \& alcohol and substance use}

Sleep-wake and circadian biology appears to be useful for characterising two functional domains in young people, namely social and economic participation, and suicide and self-harm behaviours. Three sleep-wake and circadian studies investigated the relationship between salivary cortisol secretion and social and economic participation. Two of these studies [102, 103] were longitudinal investigations that identified that increased salivary cortisol at baseline [102], and before alprazolam treatment for patients with panic disorder [103] predicted poorer social and economic participation at follow up. Such results suggest that increased HPA activity, indexed by a greater salivary cortisol response, may be indicative of HPA axis deregulation with prognostic significance and not simply a cross sectional marker of stress and active illness. Similarly, the one cross-sectional study [104] found that increased cortisol secretion during a social interaction task was associated with poorer social functioning. Alone, this study would seem to demonstrate that increased cortisol secretion is a state marker of social stress, however in light of the previous longitudinal studies, it is possible that heightened HPA activity is indicative of a persistent dysregulated stress response to social specific cues. Notably, all three of these studies were published over 15 years ago, suggesting the need for new evidence to explore the relationships identified by the longitudinal studies.

Three sleep-wake and circadian studies were longitudinal and identified a relationship with suicide outcomes in MDD patients. At baseline, higher growth hormone secretion during the first $4 \mathrm{~h}$ of sleep [105], and higher cortisol secretion in the late hours of sleep [106] were both associated with the emergence of a suicide attempt at follow-up. The final study [107] identified that reduced delta sleep activity was associated with higher levels of suicidality as well as depression severity. Similarly, to the social and economic participation studies, these biological substrates also point to HPA dysregulation as a predictor of later suicide attempts in MDD patient groups. 


\section{Functional domain: clinical syndrome}

Of the eighteen sleep-wake and circadian biology studies, nine studies investigated clinical syndrome utilising cortisol responses. Whilst, the timing of cortisol secretion varied between studies, the findings consistently indicate that increased cortisol response is associated with the development [108-110] and persistence [102, 111] of MDD, whilst remission [112] is associated with reductions in cortisol measures. The heterogeneity of depression becomes clearer with evidence of moderate-to-severe depression being associated with significant blunting of the cortisol response compared to those with mild depression, who had increased cortisol secretion during a stress task [113]. These results suggest that the neurobiological systems mediating the stress response are functioning very differently, and may indicate distinct pathophysiological drivers of depression for these two groups. Specifically, genetically mediated depression associated with increased severity and chronic stress leading to a desensitization of glucocorticoid receptors versus mild to moderate depression arising from predominately environment risk factors with typical HPA abnormalities [113]. This pattern of findings appears to differ for comorbid MDD and anxiety, which was associated with flatter diurnal cortisol slopes (daytime cortisol activity) [114], suggesting that the presence of anxiety influences cortisol function in a way that contrasts to depression alone.

Studies that investigated the relationship between the sleep-wake cycle and clinical syndrome reported quite consistent findings. When compared to unipolar depression, bipolar disorder is associated with delayed onset and lower levels of melatonin secretion [115], as well as increased rates of delayed sleep [116]. Similarly, poor sleep efficiency and lower sleep duration was reported in both unipolar depression [117] and hypomanic individuals [118] compared to healthy controls. Increased depression severity was associated with reduced delta sleep [107], while higher overnight dissipation of slow wave sleep predicted a reduction in depressive symptoms [119]. Similarly, greater high density REM and lower REM latency at baseline was associated with the development of depression at follow in healthy controls [111], whilst another found that greater high density REM was associated with fluoxetine treatment in patients with MDD [120]. Whilst these sleep-wake cycle deficits are evident in those with a full threshold disorders (both unipolar and bipolar disorder), increased rates of delayed sleep are also evident in individuals with either a discrete disorder or an attenuated syndrome compared to individuals with mild symptoms and healthy controls [121]. This indicates that a similar pattern of sleep-wake deficit are also evident in those with subthreshold disorders and highlight that such deficits can be identified earlier in the course of illness.

\section{Neurophysiology}

There were 21 studies (a total of 2034 participants; $69.6 \%$ female) that utilised neurophysiology and across these studies $74 \%(1510 / 2034)$ were patients and $26 \%$ $(524 / 2034)$ were healthy controls. Among the patient group $56 \%$ (851/1510) had depression, $9 \%(130 / 1510)$ had bipolar, $21 \%(313 / 1510)$ had anxiety, and $14 \%$ $(216 / 2034)$ were classified as other.

\section{Functional domains: social and economic participation, physical health, suicide and self-harm \& alcohol and substance use}

Evidence from the included studies indicated that neurophysiology may be particularly useful for characterising suicide and self-harm behaviours, and alcohol and substance use. Three separate neurophysiological studies found an association with specific types of suicide and self-harm behaviours. Specifically, lower contingent negative variation was associated with multiple episodes of deliberate self-harm [122], increased posterior EEG alpha asymmetry was associated with suicidal intent and the lethality of suicide attempt, but not depression severity, in suicide attempters [123], and slower reaction times during incentive based decision making tasks was associated with increased frequency for suicide and selfharm behaviours in remitted MDD patients [124]. Together these findings link abnormal brain functions implicated in decision making processes to different types of self-harm and suicidal behaviours [125, 126].

\section{Functional domain: clinical syndrome}

With regard to the fifteen neurophysiology studies, a number of these examined the relationship between treatment response and neurophysiological markers using a number of methods. Firstly, increased startle response was associated with multiple episodes of depression in depressed individuals [127]. Increased startle response was also associated with the presence of an anxiety disorder compared to healthy controls [128]. In this study, a reduction in acoustic startle response was associated with a reduction in anxiety symptoms following CBT, and a higher startle response at baseline predicted treatment response. Similarly, steep N1 of the Loudness Dependency of Auditory Evoked Potentials (LDAEP) at baseline and higher P2 LDAEP at week 1 predicted anti-depressant treatment response in patients with MDD [129]. These findings are consistent with the phenomenon described in this paper (see 'Neuroimaging') that link maladaptive processes associated with increased threat processing measured at a neural, psychological and behavioural level [130]. These findings implicate the amygdala as the predominate brain region involved in threat processing, however it functions as 
part of a wider brain circuit involving the dorsal medial prefrontal (anterior cingulate) cortex [131, 132].

Four studies utilised Event Related Negativity (ERN) to characterise the clinical syndrome of OCD patient groups. All four studies found that increased ERN was associated with OCD compared to healthy controls [133-136], however none identified a relationship with symptoms severity, treatment status or medication use. Conversely the N100 ERP had an inverse relationship with symptom severity, whilst both the N100 and P200 had an inverse relationship with illness chronicity [52]. These studies indicate that ERN may be a trait measure associated with OCD independent of symptoms severity, diagnosis and/or treatment effects, while the ERP N100 and P200 may be measures sensitive to illness specific factors (e.g. chronicity).

\section{Metabolic}

There were 10 studies (a total of 1,385 participants; $43 \%$ female) that utilised metabolic measures and across these studies $80 \%(1133 / 1385)$ were patients and $18 \%$ $(252 / 1385)$ were healthy controls. Among the patient group $22 \%(254 / 1133)$ had depression, $35 \%(400 / 1133)$ had bipolar, $6 \%(72 / 1133)$ had anxiety, and $36 \%$ (407/ 1133) were classified as other.

\section{Functional domains: social and economic participation, physical health, suicide and self-harm \& alcohol and substance use}

Three metabolic studies investigated the relationship between suicide and self-harm behaviours and cholesterol. Lower total cholesterol levels were associated with a suicide attempt history [137], and increased severity of suicidal behaviour (e.g. ideations, gestures) among those who were currently suicidal [138], while lower high density lipid cholesterol was associated with higher suicide ideation [139]. Together these findings seem to indicate that lower cholesterol is associated with the spectrum of suicidal behaviours, particularly in males. However, this is an area of contention since higher total cholesterol was associated with current suicidal behaviour versus those not currently suicidal. These findings highlight the complex association between suicidal behaviours and cholesterol, and indicate that its usefulness a biological marker needs further clarification.

Two studies examined the correlates of the risk of obesity, indexed by BMI, among young people with mood disorders. Substance use was associated with an 2.8 fold increased risk of overweight/obesity among bipolar patients and increased BMI was associated with better social and economic participation over a 4-year longitudinal study in mood disorder patients (unipolar and bipolar) [140]. The latter study also reported an increase in the prevalence rates for overweight/obesity, consistent with the finding of the former study. There are a number of illness related factors that could explain this relationship which include a return of normal appetite, medication use, self-modulation of mood by overeating [141] as well as biological factors implicated in mood, and metabolic function and weight maintenance, such as leptin [142] and neurotransmitter abnormalities [143].

\section{Functional domain: clinical syndrome}

See Table 7 for individual results.

\section{Discussion}

As expected, there is a predominate focus in the literature on clinical syndrome in young patients compared to the other four functional domains (i.e. social and economic participation, physical health, suicide and selfharm behaviours and alcohol and substance use). Whilst the neurobiology of these clinical syndromes have been extensively reviewed previously [131, 144], we provide an overview of these findings. Typically biomarkers of the clinical syndrome alone do not readily provide a complete understanding of disability and the risk factors that put young people at greater risk for a worse illness trajectory [3]. This review demonstrates the use of these biomarkers to investigate multiple functional domains in addition to the clinical syndrome. It is clear that the nature of the relationship between the underlying neurobiology and functional domains is an issue that needs to be resolved in this area. However, overall this review exhibits the usefulness of neurobiological parameters to assess these additional functional domains and identify treatment targets, in addition to the traditional focus on clinical syndrome, to optimise interventions and improve illness trajectories.

\section{Implications for personalised psychiatry}

The search for gold standard screening or diagnostic tests has ultimately been unsuccessful, however the increasing emphasis on personalised (or 'stratified') psychiatry has the potential to make significant advances in terms of clinical validity and applicability [145]. Whilst, conventional diagnostic methods remain entirely relevant, the addition of the neurobiological markers that indicate prognosis or potential treatment targets are essential for advancing personalised psychiatry. Utilising individual characteristics to guide treatment decisions is key to personalised medicine and providing personcentred care. This review exhibits the utility of the $\mathrm{RDoC}$ approach to investigate individual characteristics that extend to functional domains that contribute to ongoing disability and poorer outcomes. By collating the 
Alcohol and substance use

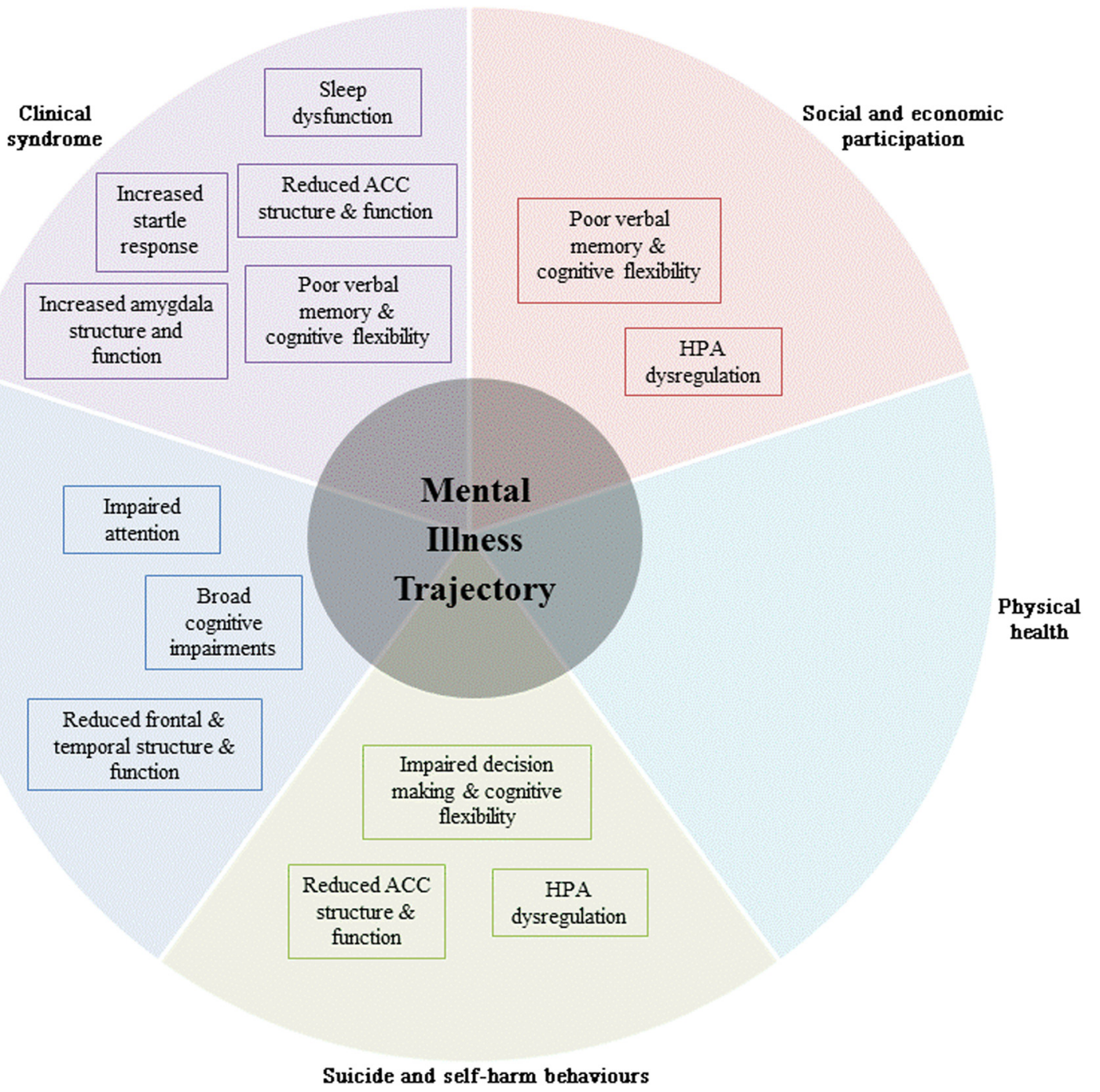

Suicide and self-harm behaviours

Fig. 2 The potential neurobiological targets associated with mental illness trajectory organised by functional domain. This is a model illustrating the potential neurobiological targets for each functional domain identified by this systematic review. At the centre is 'mental illness trajectory' that is a term employed here to encompass all the characteristic features of illness, and its associated short term and long term outcomes. Surrounding this are the five functional domains investigated by this review to illustrate how a young person's mental illness trajectory is made up of and influenced by each of these functional domains. Within the respective sections of the figure, the potential neurobiological targets for each functional domain identified by this systematic review are highlighted in the boxes. The absence of any neurobiological targets in the physical health section of the figure reflects the lack of investigation into this particular functional domain emphasising the need for more research into this area. The clinical syndrome section is the most populated which reflects the predominant focus in the literature on this particular domain. Despite less focus on the remaining three functional domains, the evidence for potential neurobiological targets for these areas is promising for clinical utility and future research

available evidence, this systematic review provides a basis for future investigations to further evaluate the clinical utility of specific neurobiological markers and their relationship with these functional domains (Fig. 2).

\section{Social and economic participation}

Our findings suggest that those with more severe impairments in memory and executive functioning are at the greatest risk for diminished participation in education, employment and social settings. The use of cognitive tests that characterise memory and executive functions of young people with emerging illnesses may be particularly useful for identifying individuals who are at risk of poor social and economic participation outcomes. Unlike diagnosis, neuropsychological function independently predicts social and economic participation outcomes in young people with emerging mood disorders [146]. The evidence suggests that neuropsychology, specifically memory and executive function, may mediate these outcomes in young people. Therefore, 
those with identified weaknesses in these areas are likely to require more intensive intervention targeted at these deficits to improve this functional domain. Such treatment practise has demonstrated clinical utility for improving memory performance in adult groups with affective disorders [147]. Moreover, cognitive training was comparable to pharmacological treatment for improving the depression state, whilst also associated with additional benefits such as, no adverse side effects, improved levels of anxiety, and better academic performance [148]. Cognitive training may slow or prevent the impact of cognitive impairment on the social and economic participation domain for young people with an emerging affective disorder.

Notably, the common indicators of social and economic functioning in the reviewed studies varied greatly with some measures (e.g. SOFAS, academic grades) being more useful than others (e.g. GAF) that conflate symptoms and functioning. Of the reviewed studies, there was a lack of focus on social functioning and the relationship with certain neurobiological parameters. The only study to specifically examine this relationship identified that HPA dysregulation, indexed by an enhanced cortisol response during a social task was predictive of social functioning [104]. Although the aforementioned study was cross sectional, longitudinal evidence supporting the predictive validity of HPA dysregulation and greater disability (including social disability), provides further support for the role of HPA dysregulation in social and economic participation [102, 103]. However, it is clear that the role of HPA functioning is complicated given its implications in multiple functional domains including suicide and self-harm behaviours and clinical syndrome.

\section{Physical health}

Physical health as a functional domain for young people with mood disorders has been notably understudied (only three studies met the inclusion criteria) compared to the other four functional domains. This is not completely unexpected considering that the majority of neurobiological modalities (neuropsychology, neuroimaging, sleep-wake and circadian biology and neurophysiology) are not recognised as being the traditional method for investigating this particular domain [149]. Of note, given that metabolic measures are used to classify physical health outcomes (i.e. BMI is both a physical health outcome and a metabolic measure) it was not deemed appropriate to carry out searches for metabolic measures and physical health outcomes. Future studies may look to improve the methods and/or key terms used to investigate the best available measures to assess and track physical health outcomes in this population.

\section{Suicide and self-harm behaviours}

Studies investigating suicide and self-harm behaviours spanned across all five neurobiological parameters, and yielded consistent findings across these parameters. Across studies, those with specific executive function impairments in the domains of decisionmaking and conceptual flexibility appear to be more likely to engage in suicidal thinking or behaviours. Therefore, identifying these deficits may be particularly important for recognising at risk patients and providing effective interventions. Furthermore, our findings provide converging evidence from different measures of brain structure and function that suicide and self-harm behaviours are associated with significant disruptions in decision-making ability. The reduced ACC volumes and activity in the identified neuroimaging studies as well as neurophysiological evidence of lower accuracy at differentiating correct and incorrect responses on a decision-making task corroborate the neuropsychological findings regarding the relationship between suicide and self-harm behaviours and impaired decision making and conceptual flexibility.

Importantly, these findings have major implications for the assessment and intervention of suicide and self-harm behaviours. Firstly, decision making ability should be incorporated into the assessment of young people with mood and anxiety disorders to stratify young people on the basis of risk for suicidal and self-harms behaviours. Whilst, there are certainly other risk factors involved in suicide and self-harm behaviours [150, 151], the findings from this review indicate that decision-making may be a mediator of suicide and self-harm outcomes. Secondly, cognitive remediation interventions aimed at improving decision-making ability in those young people that are identified as having significant impairments may prove to be a successful early intervention to reduce or prevent elevated risk for suicide and self-harm behaviours [152].

The relationship between metabolic studies investigating blood cholesterol and suicide and self-harm behaviours was another major area identified by this review. Three studies investigating cholesterol consistently identified an association between suicide and self-harm behaviours and lower cholesterol levels. Such findings are consistent with evidence that decreased cholesterol levels in the brain may be associated with reduced synaptic plasticity and impaired neurobiological functioning [153]. Furthermore, lower serotoninergic activity has also been associated with reduced cholesterol and implicated in the affective disorders [154], which was evident in another metabolic study identified by this review [155]. It has been 
suggested that these changes to cholesterol levels may be the result of HPA axis dysfunction, however further studies are needed to explore these associations and investigate the treatment implications.

\section{Alcohol and substance use}

Converging evidence from neuropsychology, neuroimaging and neurophysiology indicate that alcohol use is associated with global impairments. Unsurprisingly, all the neuroimaging studies suggest that widespread impairment across frontal and temporal areas of the brain are associated with alcohol and substance use. Specifically, reduced brain volume and function are particularly prominent in these areas and these results reflect the findings of neuropsychological and neurophysiology studies that suggest alcohol and substance use to be associated with cognitive impairments and poor attention. Independently each individual study's limitation of small sample sizes and lack of replication limit their clinical applicability, however together the evidence points to similar phenomena.

An important issue is to determine the how these findings can be used to understand the risk factors associated with alcohol and substance use. Many of the impairments may be the result of alcohol and substance use rather than a mediating factor involved in the risk of engaging in these behaviours. These problems have major implications in terms of assessment and intervention. Whilst, the aforementioned neuroimaging, neuropsychology and neurophysiology studies may be useful for tracking changes in the effects of these behaviours overtime, it is still unclear what assessment measures may be particularly useful in the early identification of individuals at risk for engaging in these harmful behaviours. More specific longitudinal studies before young people engage in alcohol and substance use are needed to differentiate between pre-existing and subsequent effects of alcohol and substance use. These longitudinal investigations will help model the role that risk factors such as risk taking, impulsivity, social occupational factors and decisionmaking play in the development of poorer alcohol and substance use outcomes.

\section{Clinical syndrome}

The final functional domain addressed by this review has been extensively reviewed elsewhere [131, 144], and therefore we provide an overview of these findings specific to young people and in the context of the other four functional domains. The primary focus of this particular domain is to identify features of the clinical syndrome that may help characterise a young person's illness phenotype and/or stage of illness $[25,156]$. The majority of studies identified by this review utilised case- control methods to investigate the neurobiological characteristics that separate discrete diagnostic cases from healthy controls, however these distinctions often provide limited clinically useful information. Those studies that sought to describe how specific neurobiological characteristics are related to illness severity (or perhaps more importantly, employed a case-case control method to delineate between cases) were particularly useful for identifying neurobiological or neurocognitive risk factors of poorer illness trajectories. Using a multi parameter approach, this review has been able to collate evidence that covers a number of 'columns' in the RDoC matrix (i.e. circuits, physiology, behaviours) to study the pathophysiological drivers of each functional domain, which is crucial for identifying treatment targets [157].

This review has provided evidence to indicate that verbal memory problems may be an early indicator associated with the emergence of depression since these deficits were evident in young people with depression, not identified as MDD, and the emergence of depressive symptoms in a community sample) [44, 45]. However, for those young people with more persistent MDD and more severe depression or anxiety symptoms executive function deficits seem to be more prominent. Similarly, another line of evidence emerging from this review indicates that greater illness severity is associated with greater reductions in the ACC, a prefrontal brain region associated with executive function. Considering the previously discussed results regarding the relationship between executive function, and another two functional domains (i.e. social and economic participation and suicide and self-harm behaviours), this reiterates the role of executive function as a mediating factor that is associated with poorer illness outcomes across multiple functional domains that should be a clear treatment target at both a brain circuit and behaviour level. The benefits of targeting neuropsychological function are made clear by treatment studies that have shown that improved cognitive function following a form of treatment, namely, TMS or pharmacological, has improve hallmark symptoms in both bipolar and depression. Again, this is reiterated by evidence from neuroimaging that treatments increasing ACC function are associated with clinical improvements in depression and bipolar.

The role of comorbid anxiety in depression and bipolar disorder is notable and associated with a substantial increase in morbidity and mortality [158-160]. From a circuitry point of view, the amygdala is one of the primary brain regions in a broader network that is involved in depression and anxiety [131, 132]. Specifically, evidence from this review indicates that increased amygdala volumes are associated with increased anxiety symptom 
severity in MDD as well as a diagnosis of GAD, reiterating theories that these conditions share similar pathophysiology that cuts across diagnostic boundaries [161]. Importantly, other neuroimaging work utilising fMRI has demonstrated that heightened amygdala activity is associated with both depression and anxiety [132]. This is evidenced by the neurophysiology study identified by this review whereby successful CBT treatment for anxiety disorder was associated with a reduction in anxiety symptoms and the startle response, another index of amygdala reactivity [128]. Together these findings implicate the amygdala as a treatment target that may reduce neurobiological substrates of anxiety, commonly implicated in the emergence of depression and a problematic feature in bipolar disorder. To reiterate the value of amygdala reactivity as a treatment target for anxiety in affective disorders, studies in the present review demonstrated that reduced amygdala activation to negative emotional stimuli, either using pharmacological treatments or CBT, were associated with clinical improvement in both bipolar and anxiety disorders.

From the sleep-wake and circadian biology studies, sleep dysfunction and cortisol secretion have consistently demonstrated a relationship with the clinical syndrome features that allude to differential illness trajectories. For example, sleep dysfunction, characterised by a number of different sleep parameters, was not only associated with the presence of a discrete depressive or bipolar illness, but similar dysfunctions were also evident in those with hypomanic or attenuated syndromes. This is a critical finding since it presents sleep dysfunction as a primary treatment target to prevent illness progression in these affective illnesses. Similarly, increased cortisol secretion was consistently implicated in the emergence of depression [102, 108-110, 112], whilst two metabolic studies suggest that increased BMI is associated with the chronic course of mood disorders [140, 162]. Neurobiologically these findings implicate HPA dysfunction as being a core feature involved in mood disorders, and so interventions aimed at improving the deficits in these brain circuits may be useful to address these clinical outcomes [163].

\section{Moving towards greater clinical translation}

One of the clear problems identified by this systematic review is the lack of consistently used patient groups and assessment measures. For any given functional domain, multiple self-report or clinician rated scales, neuroimaging techniques, and cognitive tests were implemented that often assess the same or similar outcomes, whilst the selection of patient groups varies dramatically for each study. This fundamentally limits the capacity for strong comparisons to be made between studies, or arrive at meaningful and clinically relevant conclusions. For the field of psychiatry to make new ground regarding the underlying neurobiology of psychopathology and its associated outcomes major consolidation of the common standardised measures for these key functional domains and neurobiological parameters should be implemented. Admittedly differences in scientific or clinical motivations will affect the widespread adoption of common measures, however much like the $\mathrm{RDoC}$ initiatives focus on key neurobiological domains of interest similar efforts should be made to maximise the standardised measurement of the key functional domains of interest across multiple diagnostic groups in clinical and research settings. In doing this review, we have provided an overview of the essential functional domains and current neurobiological evidence associated with these domains, the next step will be to outline the standard measures that should be drawn upon to promote better consolidation of findings in the psychiatric and neurobiological study of mood and anxiety disorders.

The lack of emphasis on all of these key functional domains and their role in disorder onset, persistence and impact from a publication, reporting and/or research priority point of view is problematic for the clinical translation of psychiatric research. Our study highlights this pertinent issue so that future research may better account for these factors and their relationship to key neurobiological parameters and disorders to improve our understanding of how these functional domains interact or relate to mental illness trajectories.

\section{Limitations and future directions}

Some limitations of this review should be considered. First, few studies investigated a particular functional domain (i.e. physical health) or utilised particular neurobiological parameters, namely sleep-wake and circadian biology or metabolic, which limits the synthesis of these findings and caution is advised when interpreting these results. Secondly, the restricted use of search terms for the functional domains or neurobiological parameters may have limited the identification of key studies, particularly favouring studies reporting current primary disorders rather than lifetime diagnoses. Best efforts were made to be inclusive of as many studies as possible to carry out a complete overview of the literature, however future studies should look to expand on this work by adding key search terms that may have been missed to further the advancement of this growing literature focusing on the functional domains of mood and anxiety disorders in young people. While we focused on RDoC levels of analysis that correspond to 'circuits,' 'physiology', 'behaviour', and 'self-report', future studies may want to include levels of analysis that include genetics, molecules 
and cells for a deeper understanding of these neurobiological factors.

Furthermore, future researchers may want to consider including borderline personality disorder into these investigations, given its significant affective component and relationship with these primary mood and anxiety disorders. Limitations associated with the systematic review process should be considered when interpreting the present findings, namely the use of one independent researcher for study selection and lack of a systematic risk of assessment bias since these may have impacted on the reliability and validity of data synthesis. Finally, the wide age range selected for this study is an important caveat that should be addressed in future studies since there are significant developmental/neurobiological changes during this dynamic period of brain development with respect to grey (e.g. pruning) and white (myelination; connectivity) matter processes.

\section{Conclusions}

Mood and anxiety disorders are especially difficult to characterise and treat in young people (age $12-30$ years) when confounds of normal development and changing environmental influences are prominent. This review identified a predominant focus in the literature on the clinical syndrome, which in our view does not adequately address key individual characteristics, such as suicide and self-harm behaviours or alcohol and substance use, that are involved in disability and persistent illness. Based on the synthesis of results from multiple neurobiological modalities, we provide a detailed summary of how the clinical utility of neurobiological measures may be improved by focussing on personalised assessment of these additional functional outcomes. We suggest that a shift in focus towards characterising the mechanisms that underlie and/or mediate multiple functional domains will optimise personalised interventions and improve illness trajectories.

\footnotetext{
Abbreviations

ACC: anterior cingulate cortex; BMI: body mass index; CBT: cognitive behavioural therapy; DLMO: dim-light melatonin onset; DSM: diagnostic and statistical manual of mental disorders; EEG: electroencephalography; ERN: error related negativity; GABA: gamma amino butyric acid; GAF: global assessment functioning; HPA: hypothalamus-pituitary-adrenal;

ICD: international classification of diseases; IGT: lowa gambling task; LDAEP: loudness dependant auditory evoked potential; LICl: long-interval cortical inhibition; MDD: major depressive disorder; MMN: mismatch negativity; MRI: magnetic resonance imaging; MRS: magnetic resonance spectroscopy; OCD: obsessive compulsive disorder; PRISMA: Preferred Reporting Items for Systematic Reviews and Meta-Analyses; RDoC: research domain criteria; REM: rapid eye movement; SAD: social anxiety disorder; SOFAS: social occupational functioning assessment scale; SSRI: selective serotonin reuptake inhibitor; TMS: transcranial magnetic stimulation.
}

Funding

Funding for this study was provided by a National Health \& Medical Research Council (NHMRC) Centres of Research Excellence (No. 1061043; 'Optymise') grant. Fl is supported by an Optymise PhD scholarship. RSCL is supported by an Optymise post-graduate scholarship. IBH is supported by a NHMRC Senior Principal Research Fellowship (No. 1046899). The funders had no further role in study design; in the collection, analysis and interpretation of data; in the writing of the report; and in the decision to submit the paper for publication.

\section{Availability of data and materials}

The methods and results presented and discussed in this article are available in Tables 1-7.

\section{Authors' contributions}

$\mathrm{Fl}$ conducted the systematic review, and prepared the initial draft manuscript. DFH supervised FI during this process. FI, IBH and DFH, conceived the study design. $\mathrm{RSCL}, \mathrm{IBH}, J \mathrm{~L} \& \mathrm{DH}$ provided interpretation of the results. All authors contributed significantly to the writing of this manuscript, as well as having read and approved the final manuscript.

\section{Competing interests}

$\mathrm{IBH}$ is a Commissioner in Australia's new National Mental Health Commission from 2012. He was a director of headspace: the national youth mental health foundation until January 2012. He was previously the chief executive officer (till 2003) and clinical adviser (till 2006) of beyondblue, an Australian National Depression Initiative. He is the Co -Director, Health and Policy at the Brain and Mind Centre which operates two early-intervention youth services under contract to headspace. He has led a range of community-based and pharmaceutical industry-supported depression awareness and education and training programs. He has led projects for health professionals and the community supported by governmental, community agency and pharmaceutical industry partners (Wyeth, Eli Lily, Servier, Pfizer, AstraZeneca) for the identification and management of depression and anxiety. He has received honoraria for presentations of his own work at educational seminars supported by a number of non-government organisations and the pharmaceutical industry (including Servier, Pfizer, AstraZeneca, and Eli Lilly). He is a member of the Medical Advisory Panel for Medibank Private and also a Board Member of Psychosis Australia Trust. He leads an investigator-initiated study of the effects of agomelatine on circadian parameters (supported in part by Servier) and has participated in a multicentre clinical trial of the effects of agomelatine on sleep architecture in depression and a Servier-supported study of major depression and sleep disturbance in primary care settings. DFH has received honoraria for educational seminars from JanssenCilag and Eli Lilly.

\section{Consent for publication}

Not applicable.

\section{Ethics approval and consent to participate}

This study was performed in accordance with the declaration of Helsinki, and it was approved by the Tarbiat Modares Institutional ethical review board (Reference Number: 1789965214). All participants signed informed consent before the start of the study.

Received: 22 October 2015 Accepted: 8 May 2016

Published online: 23 May 2016

\section{References}

1. Collins PY, Patel V, Joestl SS, March D, Insel TR, Daar AS, Bordin IA, Costello EJ, Durkin M, Fairburn C. Grand challenges in global mental health. Nature. 2011:475(7354):27-30.

2. Gore FM, Bloem PJ, Patton GC, Ferguson J, Joseph V, Coffey C, Sawyer SM, Mathers CD. Global burden of disease in young people aged 10-24 years: a systematic analysis. Lancet. 2011;377(9783):2093-102.

3. Hickie IB, Scott J, Hermens DF, Scott EM, Naismith SL, Guastella AJ, Glozier N, McGorry PD. Clinical classification in mental health at the cross-roads: which direction next? BMC Med. 2013;11(1):125.

4. Heimberg RG. Cognitive-behavioral therapy for social anxiety disorder: current status and future directions. Biol Psychiatry. 2002;51(1):101-8.

5. Scott J, Leboyer M, Hickie I, Berk M, Kapczinski F, Frank E, Kupfer D, McGorry P. Clinical staging in psychiatry: a cross-cutting model of diagnosis with heuristic and practical value. Br J Psychiatry. 2013;202(4):243-5. 
6. Paus T, Keshavan M, Giedd JN. Why do many psychiatric disorders emerge during adolescence? Nat Rev Neurosci. 2008;9(12):947-57.

7. Carrión RE, McLaughlin D, Goldberg TE, Auther AM, Olsen RH, Olvet DM, Correll CU, Cornblatt BA. Prediction of functional outcome in individuals at clinical high risk for psychosis. JAMA Psychiatry. 2013;70(11):1133-42.

8. McGorry PD, Hickie IB, Yung AR, Pantelis C, Jackson HJ. Clinical staging of psychiatric disorders: a heuristic framework for choosing earlier, safer and more effective interventions. Aust N Z J Psychiatry. 2006;40(8):616-22.

9. Insel TR, Sahakian BJ, Voon V, Nye J, Brown VJ, Altevogt BM, Bullmore ET, Goodwin GM, Howard RJ, Kupfer DJ. Drug research: a plan for mental illness. Nature. 2012;483(7389):269.

10. Scott EM, Hermens DF, Glozier N, Naismith SL, Guastella AJ, Hickie IB. Targeted primary care-based mental health services for young Australians. Med J Aust. 2012;196(2):136-40

11. Scott J, Scott EM, Hermens DF, Naismith SL, Guastella AJ, White D, Whitwell B, Lagopoulos J, Hickie IB. Functional impairment in adolescents and young adults with emerging mood disorders. Br J Psychiatry. 2014;205(5):362-8

12. Stuart MJ, Baune BT. Depression and type 2 diabetes: inflammatory mechanisms of a psychoneuroendocrine co-morbidity. Neurosci Biobehav Rev. 2012;36(1):658-76.

13. Moran P, Coffey C, Romaniuk H, Olsson C, Borschmann R, Carlin JB, Patton GC. The natural history of self-harm from adolescence to young adulthood: a population-based cohort study. Lancet. 2012;379(9812):236-43.

14. Hawton K, Saunders KE, O'Connor RC. Self-harm and suicide in adolescents. Lancet. 2012;379(9834):2373-82.

15. De Leo D, Heller TS. Who are the kids who self-harm? An Australian selfreport school survey. Med J Aust. 2004;181(3):140-4.

16. Merikangas K, Mehta RL, Molnar BE, Walters EE, Swendsen JD, AguilarGaziola S, Bijl R, Borges G, Caraveo-Anduaga JJ, Dewit DJ. Comorbidity of substance use disorders with mood and anxiety disorders: results of the International Consortium in Psychiatric Epidemiology. Addict Behav. 1998:23(6):893-907.

17. Hermens DF, Scott EM, White D, Lynch M, Lagopoulos J, Whitwell BG, Naismith SL, Hickie IB. Frequent alcohol, nicotine or cannabis use is common in young persons presenting for mental healthcare: a crosssectional study. BMJ Open. 2013;3(2). doi: 10.1136/bmjopen-2012002229

18. WHO. International Classification of Functioning, Disability, and Health: Children \& Youth Version: ICF-CY. Geneva: World Health Organization; 2007.

19. McGorry PD, Tanti C, Stokes R, Hickie IB, Carnell K, Littlefield LK, Moran J. Headspace: Australia's National Youth Mental Health Foundation-where young minds come first. Med J Aust. 2007;187(7 Suppl):S68-70.

20. Howe D, Coates D, Batchelor S. Headspace Gosford data: The local application of a National model. Australas Psychiatry. 2014;22(4):374-7.

21. Sahakian BJ, Malloch G, Kennard C. A UK strategy for mental health and wellbeing. Lancet. 2010;375(9729):1854-5.

22. Hyman SE. The diagnosis of mental disorders: the problem of reification. Annu Rev Clin Psychol. 2010;6:155-79.

23. Buckholtz JW, Meyer-Lindenberg A. Psychopathology and the human connectome: toward a transdiagnostic model of risk for mental illness. Neuron. 2012;74(6):990-1004

24. Lee R, Hermens DF, Redoblado-Hodge MA, Naismith SL, Porter MA, Kaur M, White D, Scott EM, Hickie IB. Neuropsychological and sociooccupational functioning in young psychiatric outpatients: a longitudinal investigation. PLoS One. 2013:8(3):e58176.

25. Hickie IB, Hermens DF, Naismith SL, Guastella AJ, Glozier N, Scott J, Scott EM. Evaluating differential developmental trajectories to adolescent-onset mood and psychotic disorders. BMC Psychiatry. 2013;13(1):303.

26. Liberati A, Altman DG, Tetzlaff J, Mulrow C, Gøtzsche PC, loannidis JP, Clarke M, Devereaux P, Kleijnen J, Moher D. The PRISMA statement for reporting systematic reviews and meta-analyses of studies that evaluate health care interventions: explanation and elaboration. Ann Intern Med. 2009;151(4):W-65-94.

27. Higgins JP, Green S. Cochrane handbook for systematic reviews of interventions, vol. 5. Hoboken: Wiley Online Library; 2008.

28. Deeks JJ, Higgins J, Altman DG. Analysing Data and Undertaking MetaAnalyses. In: Cochrane Handbook for Systematic Reviews of Interventions: Cochrane Book Series. 2008. p. 243-96.

29. Korhonen V, Laukkanen E, Antikainen R, Peiponen S, Lehtonen J, Viinamaki $\mathrm{H}$. Effect of major depression on cognitive performance among treatmentseeking adolescents. Nord J Psychiatry. 2002;56(3):187-93.
30. Green MF, Kern RS, Heaton RK. Longitudinal studies of cognition and functional outcome in schizophrenia: implications for MATRICS. Schizophr Res. 2004;72(1):41-51.

31. Bridge JA, McBee-Strayer SM, Cannon EA, Sheftall AH, Reynolds B, Campo JV, Pajer KA, Barbe RP, Brent DA. Impaired decision making in adolescent suicide attempters. J Am Acad Child Adolesc Psychiatry. 2012;51(4):394-403

32. Miranda R, Gallagher M, Bauchner B, Vaysman R, Marroquin B. Cognitive inflexibility as a prospective predictor of suicidal ideation among young adults with a suicide attempt history. Depress Anxiety. 2012;29(3):180-6.

33. Oldershaw A, Grima E, Jollant F, Richards C, Simic M, Taylor L, Schmidt U. Decision making and problem solving in adolescents who deliberately selfharm. Psychol Med. 2009;39(1):95-104.

34. Pan LA, Segreti A, Almeida J, Jollant F, Lawrence N, Brent D, Phillips M. Preserved hippocampal function during learning in the context of risk in adolescent suicide attempt. Psychiatry Res. 2013;211(2):112-8.

35. Ohmann S, Schuch B, Konig M, Blaas S, Fliri C, Popow C. Self-injurious behavior in adolescent girls. Association with psychopathology and neuropsychological functions. Psychopathology. 2008;41(4):226-35.

36. Mann JJ. Neurobiology of suicidal behaviour. Nat Rev Neurosci. 2003;4(10):819-28.

37. Bechara A, Damasio AR, Damasio H, Anderson SW. Insensitivity to future consequences following damage to human prefrontal cortex. Cognition. 1994;50(1):7-15

38. Van Heeringen C, Bijttebier S, Godfrin K. Suicidal brains: a review of functional and structural brain studies in association with suicidal behaviour. Neurosci Biobehav Rev. 2011;35(3):688-98.

39. Fujii Y, Kitagawa N, Shimizu Y, Mitsui N, Toyomaki A, Hashimoto N, Kako Y Tanaka T, Asakura S, Koyama T, et al. Severity of generalized social anxiety disorder correlates with low executive functioning. Neurosci Lett. 2013;543: $42-6$.

40. Biederman J, Petty CR, Wozniak J, Wilens TE, Fried R, Doyle A, Henin A, Bateman C, Evans M, Faraone SV. Impact of executive function deficits in youth with bipolar I disorder: a controlled study. Psychiatry Res. 2011;186(1): 58-64.

41. Lee R, Redoblado-Hodge M, Naismith S, Hermens D, Porter M, Hickie I. Cognitive remediation improves memory and psychosocial functioning in firstepisode psychiatric out-patients. Psychol Med. 2013:43(06):1161-73.

42. Baune BT, Fuhr M, Air T, Hering C. Neuropsychological functioning in adolescents and young adults with major depressive disorder-A review. Psychiatry Res. 2014;218:261-71.

43. Castaneda AE, Tuulio-Henriksson A, Marttunen M, Suvisaari J, Lönnqvist J. A review on cognitive impairments in depressive and anxiety disorders with a focus on young adults. J Affect Disord. 2008;106(1):1-27.

44. Gunther T, Holtkamp K, Jolles J, Herpertz-Dahlmann B, Konrad K. Verbal memory and aspects of attentional control in children and adolescents with anxiety disorders or depressive disorders. J Affect Disord. 2004 82(2):265-9

45. Simons CJ, Jacobs N, Derom C, Thiery E, Jolles J, van Os J, Krabbendam L. Cognition as predictor of current and follow-up depressive symptoms in the general population. Acta Psychiatr Scand. 2009;120(1):45-52.

46. Bitter SM, Mills NP, Adler CM, Strakowski SM, DelBello MP. Progression of amygdala volumetric abnormalities in adolescents after their first manic episode. J Am Acad Child Adolesc Psychiatry. 2011;50(10):1017-26.

47. Cataldo MG, Nobile M, Lorusso ML, Battaglia M, Molteni M. Impulsivity in depressed children and adolescents: a comparison between behavioral and neuropsychological data. Psychiatry Res. 2005;136(2-3):123-33.

48. Han G, Klimes-Dougan B, Jepsen S, Ballard K, Nelson M, Houri A, Kumra S, Cullen K. Selective neurocognitive impairments in adolescents with major depressive disorder. J Adolesc. 2012;35(1):11-20.

49. Klimkeit El, Tonge B, Bradshaw JL, Melvin GA, Gould K. Neuropsychological deficits in adolescent unipolar depression. Arch Clin Neuropsychol. 2011; 26(7):662-76.

50. Andres S, Boget T, Lazaro L, Penades R, Morer A, Salamero M, CastroFornieles J. Neuropsychological performance in children and adolescents with obsessive-compulsive disorder and influence of clinical variables. Biol Psychiatry. 2007;61(8):946-51.

51. Andres S, Lazaro L, Salamero M, Boget T, Penades R, Castro-Fornieles J. Changes in cognitive dysfunction in children and adolescents with obsessive-compulsive disorder after treatment. J Psychiatr Res. 2008;42(6): $507-14$. 
52. Okasha A, Rafaat M, Mahallawy N, Nahas GE, Dawla A, Sayed M, Kholi SE. Cognitive dysfunction in obsessive-compulsive disorder. Acta Psychiatr Scand. 2000;101(4):281-5.

53. Basso MR, Bornstein RA, Carona F, Morton R. Depression accounts for executive function deficits in obsessive-compulsive disorder. Neuropsychiatry Neuropsychol Behav Neurol. 2001;14(4):241-5.

54. Gorwood P. Generalized anxiety disorder and major depressive disorder comorbidity: an example of genetic pleiotropy? Eur Psychiatry. 2004; 19(1):27-33.

55. Pavuluri MN, Passarotti AM, Mohammed T, Carbray JA, Sweeney JA. Enhanced working and verbal memory after lamotrigine treatment in pediatric bipolar disorder. Bipolar Disord. 2010;12(2):213-20.

56. Wall CA, Croarkin PE, McClintock SM, Murphy LL, Bandel LA, Sim LA, Sampson SM. Neurocognitive effects of repetitive transcranial magnetic stimulation in adolescents with major depressive disorder. Front Psychiatry. 2013;4:165.

57. Perlman G, Simmons AN, Wu J, Hahn KS, Tapert SF, Max JE, Paulus MP Brown GG, Frank GK, Campbell-Sills L, et al. Amygdala response and functional connectivity during emotion regulation: a study of 14 depressed adolescents. J Affect Disord. 2012;139(1):75-84.

58. Bond DJ, Lang DJ, Noronha MM, Kunz M, Torres IJ, Su W, Honer WG, Lam RW, Yatham LN. The association of elevated body mass index with reduced brain volumes in first-episode mania. Biol Psychiatry. 2011;70(4): 381-7.

59. Goodman M, Hazlett EA, Avedon JB, Siever DR, Chu KW, New AS Anterior cingulate volume reduction in adolescents with borderline personality disorder and co-morbid major depression. J Psychiatr Res. 2011:45(6):803-7.

60. Pan LA, Hassel S, Segreti A, Nau S, Brent D, Phillips M. Differential patterns of activity and functional connectivity in emotion processing neural circuitry to angry and happy faces in adolescents with and without suicide attempt. Psychol Med. 2013;43(10):2129-42.

61. Bush G, Luu P, Posner Ml. Cognitive and emotional influences in anterior cingulate cortex. Trends Cogn Sci. 2000;4(6):215-22.

62. Pan LA, Batezati-Alves SC, Almeida JR, Segreti A, Akkal D, Hassel S, Lakdawala S, Brent DA, Phillips ML. Dissociable patterns of neural activity during response inhibition in depressed adolescents with and without suicidal behavior. J Am Acad Child Adolesc Psychiatry. 2011;50(6):602-611. e603.

63. Medina KL, Nagel BJ, Park A, McQueeny T, Tapert SF. Depressive symptoms in adolescents: associations with white matter volume and marijuana use. J Child Psychol Psychiatry. 2007;48(6):592-600.

64. Jarvis K, DelBello MP, Mills N, Elman I, Strakowski SM, Adler CM. Neuroanatomic comparison of bipolar adolescents with and without cannabis use disorders. J Child Adolesc Psychopharmacol. 2008;18(6):557-63.

65. Cornelius JR, Aizenstein HJ, Hariri AR. Amygdala reactivity is inversely related to level of cannabis use in individuals with comorbid cannabis dependence and major depression. Addict Behav. 2010;35(6):644-6.

66. De Bellis MD, Narasimhan A, Thatcher DL, Keshavan MS, Soloff P, Clark DB. Prefrontal cortex, thalamus, and cerebellar volumes in adolescents and young adults with adolescent-onset alcohol use disorders and comorbid mental disorders. Alcohol Clin Exp Res. 2005;29(9):1590-600.

67. Hermens DF, Lagopoulos J, Tobias-Webb J, De Regt T, Dore G, Juckes L, Latt N, Hickie IB. Pathways to alcohol-induced brain impairment in young people: a review. Cortex. 2013;49:3-17.

68. Gabbay V, Mao X, Klein RG, Ely BA, Babb JS, Panzer AM, Alonso CM, Shungu DC. Anterior cingulate cortex gamma-aminobutyric acid in depressed adolescents: relationship to anhedonia. Arch Gen Psychiatry. 2012;69(2):139-49.

69. Pannekoek JN, van der Werff SJ, van den Bulk BG, van Lang ND, Rombouts SA, van Buchem MA, Vermeiren RR, van der Wee NJ. Reduced anterior cingulate gray matter volume in treatment-naive clinically depressed adolescents. Neurolmage Clin. 2014:4:336-42.

70. Reynolds S, Carrey N, Jaworska N, Langevin LM, Yang XR, Macmaster FP. Cortical thickness in youth with major depressive disorder. BMC Psychiatry. 2014;14:83.

71. Lagopoulos J, Hermens DF, Naismith SL, Scott EM, Hickie IB. Frontal lobe changes occur early in the course of affective disorders in young people. BMC Psychiatry. 2012;12(1):4.

72. Yucel K, McKinnon MC, Chahal R, Taylor VH, Macdonald K, Joffe R, MacQueen GM. Anterior cingulate volumes in never-treated patients with major depressive disorder. Neuropsychopharmacology. 2008;33(13):3157-63.
73. Rosso IM, Cintron CM, Steingard RJ, Renshaw PF, Young AD, Yurgelun-Todd DA. Amygdala and hippocampus volumes in pediatric major depression. Biol Psychiatry. 2005;57(1):21-6.

74. MacMillan S, Szeszko PR, Moore GJ, Madden R, Lorch E, Ivey J, Banerjee SP, Rosenberg DR. Increased amygdala: hippocampal volume ratios associated with severity of anxiety in pediatric major depression. J Child Adolesc Psychopharmacol. 2003;13(1):65-73.

75. Schienle A, Ebner F, Schafer A. Localized gray matter volume abnormalities in generalized anxiety disorder. Eur Arch Psychiatry Clin Neurosci. 2011; 261(4):303-7.

76. Kendler KS, Neale MC, Kessler RC, Heath AC, Eaves L. Major depression and generalized anxiety disorder: same genes,(partly) different environments? Arch Gen Psychiatry. 1992;49(9):716.

77. Roy M-A, Neale M, Pedersen N, Mathe A, Kendler K. A twin study of generalized anxiety disorder and major depression. Psychol Med. 1995; 25(05):1037-49

78. Meng Y, Lui S, Qiu C, Qiu L, Lama S, Huang X, Feng Y, Zhu C, Gong Q, Zhang W. Neuroanatomical deficits in drug-naive adult patients with generalized social anxiety disorder: a voxel-based morphometry study. Psychiatry Res. 2013;214(1):9-15.

79. Rosenberg DR, Benazon NR, Gilbert A, Sullivan A, Moore GJ. Thalamic volume in pediatric obsessive-compulsive disorder patients before and after cognitive behavioral therapy. Biol Psychiatry. 2000;48(4):294-300.

80. Zuo C, Ma Y, Sun B, Peng S, Zhang H, Eidelberg D, Guan Y. Metabolic imaging of bilateral anterior capsulotomy in refractory obsessive compulsive disorder: an FDG PET study. J Cereb Blood Flow Metab. 2013;33(6):880-7.

81. Huyser C, van den Heuvel OA, Wolters LH, de Haan E, Boer F, Veltman DJ. Increased orbital frontal gray matter volume after cognitive behavioural therapy in paediatric obsessive compulsive disorder. World J Biol Psychiatry. 2013:14(4):319-31.

82. Zarei M, Mataix-Cols D, Heyman I, Hough M, Doherty J, Burge L, Winmill L, Nijhawan S, Matthews PM, James A. Changes in gray matter volume and white matter microstructure in adolescents with obsessive-compulsive disorder. Biol Psychiatry. 2011;70(11):1083-90.

83. MacMaster FP, Russell A, Mirza Y, Keshavan MS, Banerjee SP, Bhandari R, Boyd C, Lynch M, Rose M, Ivey J, et al. Pituitary volume in pediatric obsessive-compulsive disorder. Biol Psychiatry. 2006;59(3):252-7.

84. Rosenberg DR, Keshavan MS, Dick EL, Bagwell WW, MacMaster FP, Birmaher B. Corpus callosal morphology in treatment-naive pediatric obsessive compulsive disorder. Prog Neuropsychopharmacol Biol Psychiatry. 1997;21(8):1269-83.

85. Chang KD, Wagner C, Garrett A, Howe M, Reiss A. A preliminary functional magnetic resonance imaging study of prefrontal-amygdalar activation changes in adolescents with bipolar depression treated with lamotrigine. Bipolar Disord. 2008;10(3):426-31.

86. Phan KL, Coccaro EF, Angstadt M, Kreger KJ, Mayberg HS, Liberzon I, Stein MB. Corticolimbic brain reactivity to social signals of threat before and after sertraline treatment in generalized social phobia. Biol Psychiatry. 2013;73(4):329-36.

87. McClure EB, Adler A, Monk CS, Cameron J, Smith S, Nelson EE, Leibenluft E, Ernst $M$, Pine DS. FMRI predictors of treatment outcome in pediatric anxiety disorders. Psychopharmacology (Berl). 2007;191(1):97-105.

88. Wegbreit E, Ellis JA, Nandam A, Fitzgerald JM, Passarotti AM, Pavuluri MN, Stevens MC. Amygdala functional connectivity predicts pharmacotherapy outcome in pediatric bipolar disorder. Brain Connectivity. 2011;1(5):411-22.

89. Hariri AR, Drabant EM, Munoz KE, Kolachana BS, Mattay VS, Egan MF, Weinberger DR. A susceptibility gene for affective disorders and the response of the human amygdala. Arch Gen Psychiatry. 2005;62(2): $146-52$.

90. Aghajani M, Veer IM, van Lang ND, Meens PH, van den Bulk BG, Rombouts SA, Vermeiren RR, van der Wee NJ: Altered white-matter architecture in treatment-naive adolescents with clinical depression. Psychol Med. 2013:112. Epub ahead of print.

91. Gilbert AR, Moore GJ, Keshavan MS, Paulson LAD, Narula V, Mac Master FP, Stewart CM, Rosenberg DR. Decrease in thalamic volumes of pediatric patients with obsessive-compulsive disorder who are taking paroxetine. Arch Gen Psychiatry. 2000;57(5):449-56.

92. Lagopoulos J, Hermens DF, Hatton SN, Tobias-Webb J, Griffiths K, Naismith SL, Scott EM, Hickie IB. Microstructural white matter changes in the corpus callosum of young people with bipolar disorder: a diffusion tensor imaging study. PLoS One. 2013;8(3):e59108. 
93. Huang H, Gundapuneedi T, Rao U. White matter disruptions in adolescents exposed to childhood maltreatment and vulnerability to psychopathology. Neuropsychopharmacology. 2012;37(12):2693-701.

94. Gruner P, Vo A, Ikuta T, Mahon K, Peters BD, Malhotra AK, Ulug AM, Szeszko PR. White matter abnormalities in pediatric obsessive-compulsive disorder. Neuropsychopharmacology. 2012;37(12):2730-9.

95. Henderson SE, Johnson AR, Vallejo Al, Katz L, Wong E, Gabbay V. A preliminary study of white matter in adolescent depression: relationships with illness severity, anhedonia, and irritability. Front Psychiatry. 2013:4:152

96. Lagopoulos J, Hermens D, Hatton S, Battisti R, Tobias-Webb J, White D, Naismith S, Scott E, Ryder W, Bennett M. Microstructural white matter changes are correlated with the stage of psychiatric illness. Translat Psychiatry. 2013;3(4):e248

97. Bustillo JR. Use of proton magnetic resonance spectroscopy in the treatment of psychiatric disorders: a critical update. Dialogues Clin Neurosci. 2013;15(3):329-37.

98. Lazaro L, Bargallo N, Andres S, Falcon C, Morer A, Junque C, Castro-Fornieles J. Proton magnetic resonance spectroscopy in pediatric obsessive-compulsive disorder: longitudinal study before and after treatment. Psychiatry Res. 2012; 201(1):17-24

99. Chu WJ, Delbello MP, Jarvis KB, Norris MM, Kim MJ, Weber W, Lee JH, Strakowski SM, Adler CM. Magnetic resonance spectroscopy imaging of lactate in patients with bipolar disorder. Psychiatry Res. 2013; 213(3):230-4

100. Patel NC, Cecil KM, Strakowski SM, Adler CM, DelBello MP. Neurochemical alterations in adolescent bipolar depression: a proton magnetic resonance spectroscopy pilot study of the prefrontal cortex. J Child Adolesc Psychopharmacol. 2008;18(6):623-7

101. Strawn JR, Patel NC, Chu WJ, Lee JH, Adler CM, Kim MJ, Bryan HS, Alfieri DC, Welge JA, Blom TJ, et al. Glutamatergic effects of divalproex in adolescents with mania: a proton magnetic resonance spectroscopy study. J Am Acad Child Adolesc Psychiatry. 2012;51(6):642-51.

102. Goodyer IM, Herbert J, Altham PM. Adrenal steroid secretion and major depression in 8- to 16-year-olds, III. Influence of cortisol/DHEA ratio at presentation on subsequent rates of disappointing life events and persistent major depression. Psychol Med. 1998;28(2):265-73.

103. Abelson JL, Curtis GC. Hypothalamic-pituitary-adrenal axis activity in panic disorder: prediction of long-term outcome by pretreatment cortisol levels. Am J Psychiatry. 1996;153(1):69-73.

104. Granger DA, Weisz JR, Kauneckis D. Neuroendocrine reactivity, internalizing behavior problems, and control-related cognitions in clinic-referred children and adolescents. J Abnorm Psychol. 1994;103(2):267-76.

105. Coplan JD, Wolk SI, Goetz RR, Ryan ND, Dahl RE, Mann JJ, Weissman MM. Nocturnal growth hormone secretion studies in adolescents with or without major depression re-examined: integration of adult clinical followup data. Biol Psychiatry. 2000;47(7):594-604.

106. Mathew SJ, Coplan JD, Goetz RR, Feder A, Greenwald S, Dahl RE, Ryan ND, Mann JJ, Weissman MM. Differentiating depressed adolescent $24 \mathrm{~h}$ cortisol secretion in light of their adult clinical outcome. Neuropsychopharmacology. 2003;28(7):1336-43.

107. McCracken JT, Poland RE, Lutchmansingh P, Edwards C. Sleep electroencephalographic abnormalities in adolescent depressives: effects of scopolamine. Biol Psychiatry. 1997;42(7):577-84.

108. Adam EK, Doane LD, Zinbarg RE, Mineka S, Craske MG, Griffith JW. Prospective prediction of major depressive disorder from cortisol awakening responses in adolescence. Psychoneuroendocrinology. 2010;35(6):921-31

109. Ellenbogen MA, Hodgins S, Walker CD, Couture S, Adam S. Daytime cortisol and stress reactivity in the offspring of parents with bipolar disorder. Psychoneuroendocrinology. 2006;31(10):1164-80.

110. Ellenbogen MA, Santo JB, Linnen AM, Walker CD, Hodgins S. High cortisol levels in the offspring of parents with bipolar disorder during two weeks of daily sampling. Bipolar Disord. 2010;12(1):77-86.

111. Rao U, Dahl RE, Ryan ND, Birmaher B, Williamson DE, Giles DE, Rao R, Kaufman J, Nelson B. The relationship between longitudinal clinical course and sleep and cortisol changes in adolescent depression. Biol Psychiatry. 1996:40(6):474-84.

112. Rao U, Poland RE. Electroencephalographic sleep and hypothalamicpituitary-adrenal changes from episode to recovery in depressed adolescents. J Child Adolesc Psychopharmacol. 2008;18(6):607-13.
113. Harkness KL, Stewart JG, Wynne-Edwards KE. Cortisol reactivity to social stress in adolescents: role of depression severity and child maltreatment. Psychoneuroendocrinology. 2011;36(2):173-81.

114. Doane LD, Mineka S, Zinbarg RE, Craske M, Griffith JW, Adam EK. Are flatter diurnal cortisol rhythms associated with major depression and anxiety disorders in late adolescence? The role of life stress and daily negative emotion. Dev Psychopathol. 2013;25(3):629-42.

115. Robillard R, Naismith S, Rogers N, Scott E, Ip T, Hermens D, Hickie I. Sleepwake cycle and melatonin rhythms in adolescents and young adults with mood disorders: Comparison of unipolar and bipolar phenotypes. Eur Psychiatry. 2013;28:412-6.

116. Robillard R, Naismith SL, Rogers NL, Ip TK, Hermens DF, Scott EM, Hickie IB. Delayed sleep phase in young people with unipolar or bipolar affective disorders. J Affect Disord. 2013;145(2):260-3.

117. Murray CB, Murphy LK, Palermo TM, Clarke GM. Pain and sleep-wake disturbances in adolescents with depressive disorders. J Clin Child Adolesc Psychol. 2012;41(4):482-90.

118. Ankers D, Jones SH. Objective assessment of circadian activity and sleep patterns in individuals at behavioural risk of hypomania. J Clin Psychol. 2009;65(10):1071-86

119. Landsness EC, Goldstein MR, Peterson MJ, Tononi G, Benca RM. Antidepressant effects of selective slow wave sleep deprivation in majo depression: a high-density EEG investigation. J Psychiatr Res. 2011;45(8): 1019-26.

120. Armitage R, Emslie G, Rintelmann J. The effect of fluoxetine on sleep EEG in childhood depression: a preliminary report. Neuropsychopharmacology. 1997;17(4):241-5.

121. Scott EM, Robillard R, Hermens DF, Naismith SL, Rogers NL, Ip TK, White D, Guastella A, Whitwell B, Smith KL. Dysregulated sleep-wake cycles in young people are associated with emerging stages of major mental disorders. Early Interv Psychiatry. 2014;10:63-70.

122. Ashton $\mathrm{CH}$, Marshall EF, Hassanyeh F, Marsh VR, Wright-Honari S. Biological correlates of deliberate self-harm behaviour: a study of electroencephalographic, biochemical and psychological variables in parasuicide. Acta Psychiatr Scand. 1994;90(5):316-23.

123. Graae F, Tenke C, Bruder G, Rotheram M-J, Piacentini J, Castro-Blanco D, Leite $P$, Towey J. Abnormality of EEG alpha asymmetry in female adolescent suicide attempters. Biol Psychiatry. 1996;40(8):706-13.

124. Pechtel P, Pizzagalli DA. Disrupted reinforcement learning and maladaptive behavior in women with a history of childhood sexual abuse: a high-density event-related potential study. JAMA Psychiatry. 2013;70(5):499-507.

125. Davis CE, Hauf JD, Wu DQ, Everhart DE. Brain function with complex decision making using electroencephalography. Int J Psychophysiol. 2011;79(2):175-83.

126. Macar F, Vidal F. The CNV peak: an index of decision making and temporal memory. Psychophysiology. 2003;40(6):950-4.

127. Vaidyanathan U, Welo EJ, Malone SM, Burwell SJ, lacono WG. The effects of recurrent episodes of depression on startle responses. Psychophysiology. 2014:51(1):103-9.

128. Bakker M, Tijssen M, Koelman J, Boer F. Normalization of the auditory startle reflex after symptom reduction in children with anxiety disorders. J Psychiatr Res. 2011;45(6):796-802.

129. Jaworska N, Blondeau C, Tessier P, Norris S, Fusee W, Blier P, Knott V. Response prediction to antidepressants using scalp and source-localized loudness dependence of auditory evoked potential (LDAEP) slopes. Prog Neuro-Psychopharmacol Biol Psychiatry. 2013;44:100-7.

130. Robinson OJ, Vytal K, Cornwell BR, Grillon C. The impact of anxiety upon cognition: perspectives from human threat of shock studies. Front Hum Neurosci. 2013;7:203

131. Price JL, Drevets WC. Neural circuits underlying the pathophysiology of mood disorders. Trends Cogn Sci. 2012;16(1):61-71.

132. Etkin A. Functional neuroanatomy of anxiety: a neural circuit perspective. In: Behavioral neurobiology of anxiety and its treatment. Berlin: Springer; 2010. p. 251-77.

133. Carrasco M, Harbin SM, Nienhuis JK, Fitzgerald KD, Gehring WJ, Hanna $\mathrm{GL}$. Increased error-related brain activity in youth with obsessivecompulsive disorder and unaffected siblings. Depress Anxiety. 2013; 30(1):39-46.

134. Carrasco M, Hong C, Nienhuis JK, Harbin SM, Fitzgerald KD, Gehring WJ, Hanna GL. Increased error-related brain activity in youth with obsessive-compulsive disorder and other anxiety disorders. Neurosci Lett. 2013;541:214-8. 
135. Hajcak G, Franklin ME, Foa EB, Simons RF. Increased error-related brain activity in pediatric obsessive-compulsive disorder before and after treatment. Am J Psychiatry. 2008;165(1):116-23.

136. Stern ER, Liu Y, Gehring WJ, Lister JJ, Yin G, Zhang J, Fitzgerald KD, Himle JA, Abelson $J$, Taylor SF. Chronic medication does not affect hyperactive error responses in obsessive-compulsive disorder. Psychophysiology. 2010;47(5): 913-20

137. Plana T, Gracia R, Mendez I, Pintor L, Lazaro L, Castro-Fornieles J. Total serum cholesterol levels and suicide attempts in child and adolescent psychiatric inpatients. Eur Child Adolesc Psychiatry. 2010; 19(7):615-9.

138. Apter A, Laufer N, Bar-Sever M, Har-Even D, Ofek H, Weizman A. Serum cholesterol, suicidal tendencies, impulsivity, aggression, and depression in adolescent psychiatric inpatients. Biol Psychiatry. 1999;46(4):532-41.

139. De Berardis D, Campanella D, Serroni N, Moschetta FS, Di Emidio F, Conti C, Carano A, Acciavatti T, Di lorio G, Martinotti G. Alexithymia, suicide risk and serum lipid levels among adult outpatients with panic disorder. Compr Psychiatry. 2013;54(5):517-22.

140. Taylor V, Macdonald K, McKinnon MC, Joffe RT, MacQueen GM. Increased rates of obesity in first-presentation adults with mood disorders over the course of four-year follow-up. J Affect Disord. 2008;109(1-2):127-31.

141. Kilbourne AM, Rofey DL, McCarthy JF, Post EP, Welsh D, Blow FC. Nutrition and exercise behavior among patients with bipolar disorder1. Bipolar Disord. 2007;9(5):443-52.

142. Lu X-Y, Kim CS, Frazer A, Zhang W. Leptin: a potential novel antidepressant. Proc Natl Acad Sci. 2006;103(5):1593-8.

143. Wildes JE, Marcus MD, Fagiolini A. Obesity in patients with bipolar disorder: a biopsychosocial-behavioral model. J Clin Psychiatry. 2006;67(6):904-15.

144. Chamberlain SR, Sahakian BJ. The neuropsychology of mood disorders. Curr Psychiatry Rep. 2006;8(6):458-63.

145. Kapur S, Phillips A, Insel T. Why has it taken so long for biological psychiatry to develop clinical tests and what to do about it\&quest. Mol Psychiatry. 2012;17(12):1174-9.

146. Lee R, Hermens DF, Naismith SL, Lagopoulos J, Jones A, Chitty KM, White D, Robillard R, Scott E, Hickie I. Neuropsychological and functional outcomes in recent-onset major depression, bipolar disorder and schizophrenia-spectrum disorders: a longitudinal cohort study. Transl Psychiatry. 2015;5:e555.

147. Naismith SL, Redoblado-Hodge MA, Lewis SJ, Scott EM, Hickie IB. Cognitive training in affective disorders improves memory: a preliminary study using the NEAR approach. J Affect Disord. 2010;121(3):258-62.

148. Alvarez LM, Cortés Sotres JF, León SO, Estrella J, Sánchez Sosa JJ. Computer program in the treatment for major depression and cognitive impairment in university students. Comput Hum Behav. 2008:24(3):816-26.

149. De Hert M, Cohen D, Bobes J, Cetkovich-Bakmas M, Leucht S, Ndetei DM, Newcomer JW, Uwakwe R, Asai I, Möller H-J. Physical illness in patients with severe mental disorders. II. Barriers to care, monitoring and treatment guidelines, plus recommendations at the system and individual level. World Psychiatry. 2011;10(2):138.

150. Miret M, Ayuso-Mateos JL, Sanchez-Moreno J, Vieta E. Depressive disorders and suicide: epidemiology, risk factors, and burden. Neurosci Biobehav Rev. 2013;37(10):2372-4.

151. WHO. Mental health: new understanding, new hope. Geneva: World Health Organization; 2001

152. Cáceda R, Nemeroff CB, Harvey PD. Toward an understanding of decision making in severe mental illness. 2014.

153. Pfrieger F. Cholesterol homeostasis and function in neurons of the central nervous system. Cell Mol Life Sci. 2003;60(6):1158-71.

154. Papakostas GI, Öngür D, losifescu DV, Mischoulon D, Fava M. Cholesterol in mood and anxiety disorders: review of the literature and new hypotheses. Eur Neuropsychopharmacol. 2004;14(2):135-42.

155. Tyano S, Zalsman G, Ofek H, Blum I, Apter A, Wolovik L, Sher L, Sommerfeld E, Harell D, Weizman A. Plasma serotonin levels and suicidal behavior in adolescents. Eur Neuropsychopharmacol. 2006;16(1):49-57.

156. Hickie IB, Scott EM, Hermens DF, Naismith SL, Guastella AJ, Kaur M, Sidis A, Whitwell B, Glozier N, Davenport T, et al. Applying clinical staging to young people who present for mental health care. Early Interv Psychiatry. 2013;7(1): $31-43$.

157. Insel TR, Cuthbert BN, Garvey MA, Heinssen RK, Pine DS, Quinn K, Sanislow CA, Wang PS. Research domain criteria (RDoC): toward a new classification framework for research on mental disorders. Am J Psychiatr. 2010;167(7):748-51.
158. Keller M. Follow-up and family study of anxious depression. Am J Psychiatry 1991:148(1):1512-7.

159. Joffe RT, Bagby RM, Levitt A. Anxious and nonanxious depression. Am J Psychiatr. 1993;150:1257.

160. Fava M, Rush AJ, Alpert JE, Carmin CN, Balasubramani G, Wisniewski SR, Trivedi MH, Biggs MM, Shores-Wilson K. What clinical and symptom features and comorbid disorders characterize outpatients with anxious major depressive disorder: a replication and extension. Can J Psychiatry. 2006;51(13):823-35

161. Watson D. Rethinking the mood and anxiety disorders: a quantitative hierarchical model for DSM-V. J Abnorm Psychol. 2005;114(4):522.

162. Pine DS, Goldstein RB, Wolk S, Weissman MM. The association between childhood depression and adulthood body mass index. Pediatrics. 2001; 107(5):1049-56.

163. Holsboer F. Stress, hypercortisolism and corticosteroid receptors in depression: implicatons for therapy. J Affect Disord. 2001;62(1):77-91.

164. Harvey MA, Sellman JD, Porter RJ, Frampton CM. The relationship between non-acute adolescent cannabis use and cognition. Drug Alcohol Rev. 2007; 26(3):309-19.

165. Hermens DF, Lee RS, De Regt T, Lagopoulos J, Naismith SL, Scott EM, Hickie IB. Neuropsychological functioning is compromised in binge drinking young adults with depression. Psychiatry Res. 2013;210:256-62.

166. Fleck DE, Shear PK, Madore M, Strakowski SM. Wisconsin Card Sorting Test performance in bipolar disorder: effects of mood state and early course. Bipolar Disord. 2008;10(4):539-45.

167. Kıvırcık BB, Yener GG, Alptekin K, Aydın H. Event-related potentials and neuropsychological tests in obsessive-compulsive disorder. Prog NeuroPsychopharmacol Biol Psychiatry. 2003;27(4):601-6.

168. Schmid M, Hammar A. A follow-up study of first episode major depressive disorder. Impairment in inhibition and semantic fluency-potential predictors for relapse? Front Psychol. 2013;4:633

169. Torres IJ, DeFreitas VG, DeFreitas CM, Kauer-Sant'Anna M, Bond DJ, Honer WG, Lam RW, Yatham LN. Neurocognitive functioning in patients with bipolar I disorder recently recovered from a first manic episode. J Clin Psychiatry. 2010; 71(9):1234-42

170. Ehrlich S, Noam GG, Lyoo IK, Kwon BJ, Clark MA, Renshaw PF. White matter hyperintensities and their associations with suicidality in psychiatrically hospitalized children and adolescents. J Am Acad Child Adolesc Psychiatry. 2004;43(6):770-6.

171. Ehrlich S, Breeze JL, Hesdorffer DC, Noam GG, Hong X, Alban RL, Davis SE, Renshaw PF. White matter hyperintensities and their association with suicidality in depressed young adults. J Affect Disord. 2005;86(2):281-7.

172. Chitty KM, Lagopoulos J, Hickie IB, Hermens DF. Risky alcohol use in young persons with emerging bipolar disorder is associated with increased oxidative stress. J Affect Disord. 2013;150(3):1238-41.

173. Adler CM, DelBello MP, Jarvis K, Levine A, Adams J, Strakowski SM. Voxelbased study of structural changes in first-episode patients with bipolar disorder. Biol Psychiatry. 2007:61(6):776-81.

174. Diler RS, Ladouceur CD, Segreti A, Almeida JR, Birmaher B, Axelson DA, Phillips ML, Pan LA. Neural correlates of treatment response in depressed bipolar adolescents during emotion processing. Brain Imaging Behav. 2013: 7(2):227-35

175. Forbes EE, Christopher May J, Siegle GJ, Ladouceur CD, Ryan ND, Carter CS, Birmaher B, Axelson DA, Dahl RE. Reward-related decision-making in pediatric major depressive disorder: an fMRI study. J Child Psychol Psychiatry. 2006;47(10):1031-40.

176. Forbes EE, Olino TM, Ryan ND, Birmaher B, Axelson D, Moyles DL, Dahl RE. Reward-related brain function as a predictor of treatment response in adolescents with major depressive disorder. Cogn Affect Behav Neurosci. 2010;10(1):107-18

177. Gabbay V, Ely BA, Li Q, Bangaru SD, Panzer AM, Alonso CM, Castellanos FX, Milham MP. Striatum-based circuitry of adolescent depression and anhedonia. J Am Acad Child Adolesc Psychiatry. 2013;52(6):628-641.e613.

178. Gao W, Jiao Q, Qi R, Zhong Y, Lu D, Xiao Q, Lu S, Xu C, Zhang Y, Liu $X$, et al. Combined analyses of gray matter voxel-based morphometry and white matter tract-based spatial statistics in pediatric bipolar mania. J Affect Disord. 2013;150(1):70-6.

179. Gilbert AR, Akkal D, Almeida JR, Mataix-Cols D, Kalas C, Devlin B, Birmaher B, Phillips ML. Neural correlates of symptom dimensions in pediatric obsessivecompulsive disorder: a functional magnetic resonance imaging study. J Am Acad Child Adolesc Psychiatry. 2009;48(9):936-44. 
180. Hatton SN, Lagopoulos J, Hermens DF, Naismith SL, Bennett MR, Hickie IB. Correlating anterior insula gray matter volume changes in young people with clinical and neurocognitive outcomes: an MRI study. BMC Psychiatry. 2012;12(1):45

181. Ho TC, Yang G, Wu J, Cassey P, Brown SD, Hoang N, Chan M, Connolly CG, Henje-Blom E, Duncan LG, et al. Functional connectivity of negative emotional processing in adolescent depression. J Affect Disord. 2014;155:65-74.

182. Huyser C, Veltman DJ, Wolters LH, de Haan E, Boer F. Developmental aspects of error and high-conflict-related brain activity in pediatric obsessive-compulsive disorder: a fMRI study with a Flanker task before and after CBT. J Child Psychol Psychiatry. 2011;52(12):1251-60.

183. Ladouceur CD, Farchione T, Diwadkar V, Pruitt P, Radwan J, Axelson DA, Birmaher B, Phillips ML. Differential patterns of abnormal activity and connectivity in the amygdala-prefrontal circuitry in bipolar-l and bipolarNOS youth. J Am Acad Child Adolesc Psychiatry. 2011;50(12):1289.e1272

184. Lazaro L, Caldú X, Junqué C, Bargalló N, Andrés S, Morer A, Castro-Fornieles J. Cerebral activation in children and adolescents with obsessivecompulsive disorder before and after treatment: A functional MRI study. J Psychiatr Res. 2008;42(13):1051-9.

185. Lisy ME, Jarvis KB, DelBello MP, Mills NP, Weber WA, Fleck D, Strakowski SM, Adler CM. Progressive neurostructural changes in adolescent and adult patients with bipolar disorder. Bipolar Disord. 2011;13(4):396-405.

186. Pavuluri MN, Passarotti AM, Parnes SA, Fitzgerald JM, Sweeney JA. A pharmacological functional magnetic resonance imaging study probing the interface of cognitive and emotional brain systems in pediatric bipolar disorder. J Child Adolesc Psychopharmacol. 2010; 20(5):395-406.

187. Pavuluri MN, Passarotti AM, Lu LH, Carbray JA, Sweeney JA. Double-blind randomized trial of risperidone versus divalproex in pediatric bipolar disorder: fMRI outcomes. Psychiatry Res. 2011;193(1):28-37.

188. Rauch SL, Shin LM, Dougherty DD, Alpert NM, Fischman AJ, Jenike MA. Predictors of fluvoxamine response in contamination-related obsessive compulsive disorder: a PET symptom provocation study. Neuropsychopharmacology. 2002;27(5):782-91.

189. Schneider MR, Adler CM, Whitsel R, Weber W, Mills NP, Bitter SM, Eliassen J, Strakowski SM, Delbello MP. The effects of ziprasidone on prefrontal and amygdalar activation in manic youth with bipolar disorder. Isr J Psychiatry Relat Sci. 2012;49(2):112-20.

190. Kaur M, Lagopoulos J, Lee RSC, Ward PB, Naismith SL, Hickie IB, Hermens DF. Longitudinal associations between mismatch negativity and disability in early schizophrenia-and affective-spectrum disorders. Prog NeuroPsychopharmacol Biol Psychiatry. 2013;46:161-9.

191. Jaworska N, McIntosh J, Villeneuve C, Thompson A, Fisher D, Milin R, Knott $\checkmark$. Effects of nicotine on electroencephalography and affect in adolescent females with major depressive disorder: a pilot study. J Addict Med. 2011; 5(2):123-33

192. Chitty KM, Kaur M, Lagopoulos J, Hickie IB, Hermens DF. Risky alcohol use predicts temporal mismatch negativity impairments in young people with bipolar disorder. Biol Psychol. 2014;99:60-8.

193. Ehlers CL, Phillips E, Criado JR, Gilder DA. N4 component responses to prepulse startle stimuli in young adults: relationship to alcohol dependence. Psychiatry Res. 2011;188(2):237-44.

194. Croarkin PE, Nakonezny PA, Husain MM, Port JD, Melton T, Kennard BD, Emslie GJ, Kozel FA, Daskalakis ZJ. Evidence for Pretreatment LICI Deficits Among Depressed Children and Adolescents With Nonresponse to Fluoxetine. Brain Stimul. 2014;7(2):243-51.

195. Dai Q, Feng Z. More excited for negative facial expressions in depression: Evidence from an event-related potential study. Clin Neurophysiol. 2012; 123(11):2172-9.

196. El-Badri SM, Ashton CH, Moore PB, Marsh VR, Ferrier IN. Electrophysiological and cognitive function in young euthymic patients with bipolar affective disorder. Bipolar Disord. 2001;3(2):79-87.

197. Houston RJ, Bauer LO, Hesselbrock VM. Depression and familial risk for substance dependence: a P300 study of young women. Psychiatry Res. 2003;124(1):49-62.

198. Mannie ZN, Williams C, Diesch J, Steptoe A, Leeson P, Cowen PJ. Cardiovascular and metabolic risk profile in young people at familial risk of depression. Br J Psychiatry. 2013;203(1):18-23.
199. Soreni N, Apter A, Weizman A, Don-Tufeled O, Leschiner S, Karp L, Gavish M. Decreased platelet peripheral-type benzodiazepine receptors in adolescent inpatients with repeated suicide attempts. Biol Psychiatry. 1999;46(4):484-8.

200. Goldstein BI, Birmaher B, Axelson DA, Goldstein TR, Esposito-Smythers C, Strober MA, Hunt J, Leonard H, Gill MK, lyengar S, et al. Preliminary findings regarding overweight and obesity in pediatric bipolar disorder. J Clin Psychiatry. 2008;69(12):1953-9.

\section{Submit your next manuscript to BioMed Central and we will help you at every step:}

- We accept pre-submission inquiries

- Our selector tool helps you to find the most relevant journal

- We provide round the clock customer support

- Convenient online submission

- Thorough peer review

- Inclusion in PubMed and all major indexing services

- Maximum visibility for your research

Submit your manuscript at www.biomedcentral.com/submit
) Biomed Central 